\title{
RECEMED
}

FEA 061935

OSTI

\section{Simulations of Longitudinal Beam Dynamics of Space-Charge Dominated Beams for Heavy Ion Fusion}

\author{
D. A. Callahan Miller \\ (Ph.D. Thesis)
}

December 1994

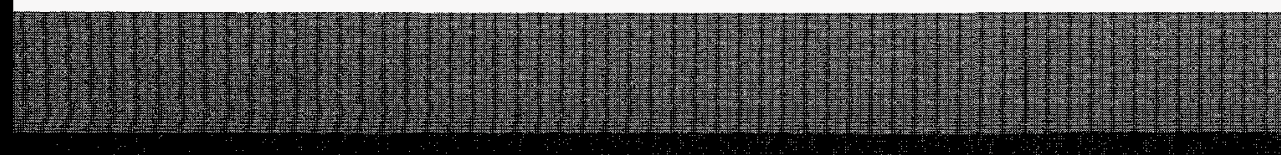

\section{MASTER}
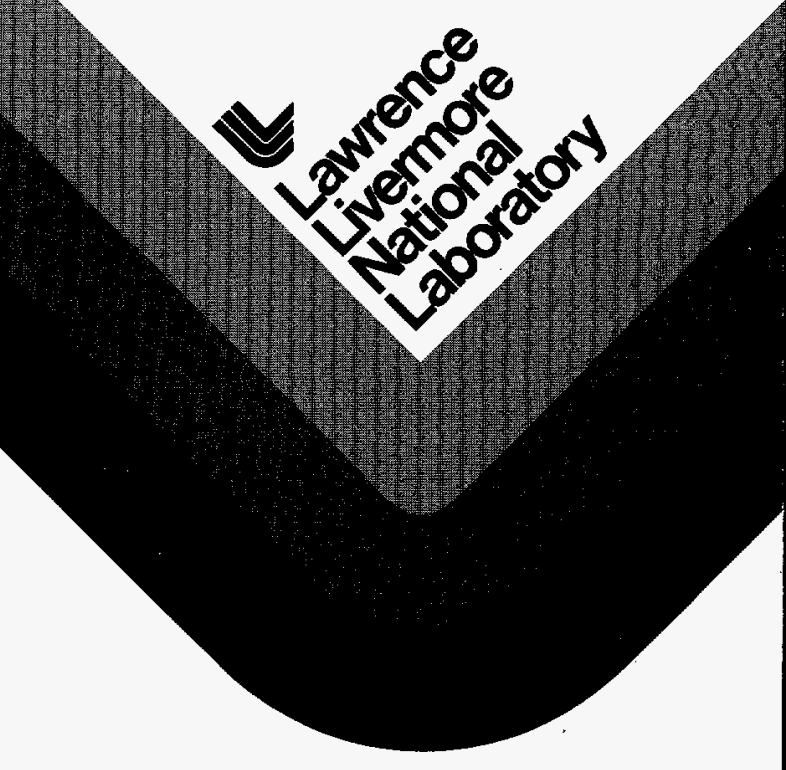

DISTRIBUTION OF THIS DOCUMENT IS UNLIMTED , 


\section{DISCLAIMER}

This document was prepared as an account of work sponsored by an agency of the United States Government. Neither the United States Government nor the University of California nor any of their employees, makes any warranty, express or implied, or assumes any legal liability or responsibility for the accuracy, completeness, or usefulness of any information, apparatus, product, or process disclosed, or represents that its use would not infringe privately owned rights. Reference herein to any specific commercial product, process, or service by trade name, trademark, manufacturer, or otherwise, does not necessarily constitute or imply its endorsement, recommendation, or favoring by the United States Government or the University of California. The views and opinions of authors expressed herein do not necessarily state or reflect those of the United States Government or the University of California, and shall not be used for advertising or product endorsement purposes.

This report has been reproduced

directly from the best available copy.

Available to DOE and DOE contractors from the Office of Scientific and Technical Information

P.O. Box 62, Oak Ridge, TN 37831

Prices available from (615) 576-8401, FTS 626-8401

Available to the public from the

National Technical Information Service

U.S. Department of Commerce

5285 Port Royal Rd.,

Springfield, VA 22161

\section{The Government reserves for itself and others acting on its behalf a royalty free, nonexclusive, irrevocable, world-wide license for governmental purposes to publish, distribute, translate, duplicate, exhibit, and perform any such data copyrighted by the contractor.}

Work performed under the auspices of the U.S. Department of Energy by Lawrence Livermore National Laboratory under Contract W-7405-ENG-48. 


\section{DISCLAMIER}

Portions of this document may be illegible in electronic image products. Images are produced from the best available original document. 


\title{
Simulations of Longitudinal Beam Dynamics of Space-Charge Dominated Beams for Heavy Ion Fusion
}

\author{
D. A. Callahan Miller \\ (Ph.D.Thesis)
}

Manuscript date: December 1994

LAWRENCE LIVERMORE NATIONAL LABORATORY University of California - Livermore, California • 94551 


\section{Simulations of Longitudinal Beam Dynamics of Space-Charge Dominated Beams for Heavy Ion Fusion}

By

Debra Ann Callahan Miller

BS, University of Denver, 1985

MS, University of California, Davis 1989

\section{DISSERTATION}

Submitted in partial satisfaction of the requirements for the degree of DOCTOR OF PHILOSOPHY

in

\section{APPLIED SCIENCE}

in the

GRADUATE DIVISION

of the

UNIVERSITY OF CALIFORNIA

DAVIS

Approved:

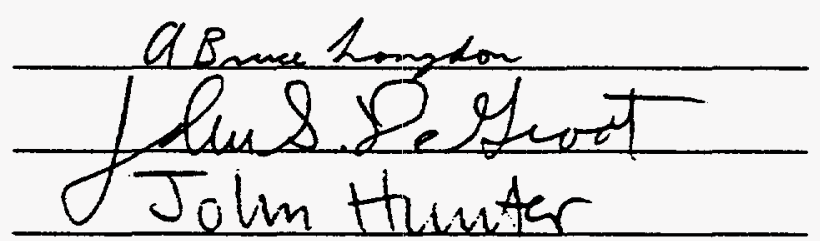

Committee in Charge

1994 
(C) Copyright 1994 by

Debra Ann Callahan Miller 


\section{Acknowledgements}

There are many people who deserve thanks for supporting me through this thesis. First, I want to thank Bruce Langdon, my advisor, for all his patient efforts to teach me how to do research. I thank John DeGroot, my academic advisor for his support, and Alex Friedman for getting me into the HIF project in the first place and for help with this thesis. I also thank Ed Lee, Tom Fessenden, and Andy Faltens from LBL, and Irv Haber from NRL for many valuable discussions.

I want to thank the physics department faculty at the University of Denver for giving me an undergraduate physics education that was as good or better than my classmates who went to larger, better known schools. I want to specifically thank Liz Tuttle who convinced me that I should go to grad school instead of teaching high school physics, and Herschel Neumann who taught me a bunch of quantum mechanics (none of which appears in this thesis) and talked me into taking my first numerical methods class.

There are many people from the Dept of Applied Science and Livermore Lab who deserve credit: the student policy committee (Charlie Westbrook, Bill Chandler, Cheryl Krossa, John Anderson and the nameless, faceless others) for funding me through this thesis, the DAS faculty, and my oral exam committee (Richard Post, Garry Rodrigue, Bill Kruer, John Hunter, and Don McQuarrie) for being gentle during the exam.

I thank my parents, Dan and Cindy Callahan, who were always encouraging, my siblings, Liz, Cori and Paul for being there, and my friends at DAS who kept me sane during the last six years. Mike and Inés Heinz, Kim Budil, Dale Slone, Dave and Jennifer Larson, Dave and Cecilia Grote, Dave and Susan Gold, Erick McCroskey, Jay 
Hartley, Cory Patrick, Brian Jones, Nick Gentile, Christine Coverdale, Jeff Latkowski and Gwen Loosmore.

Last, but certainly not least, I want to thank my husband, Doug Miller. On more than one occasion, Doug kept me working on this project by reminding me of a bargain we once made, "We have a deal. If $I$ get a $\mathrm{PhD}$, then you have to get one too." 


\section{Contents}

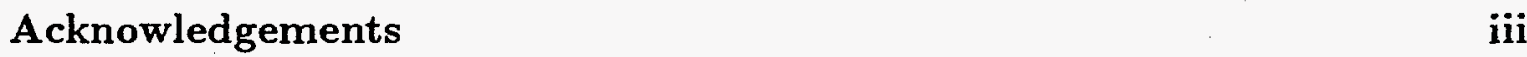

List of Figures viii

1 Introduction $\quad 3$

1.1 Heavy Ion Fusion $\ldots \ldots \ldots \ldots \ldots \ldots \ldots$

1.2 The Longitudinal Wall Impedance Instability . . . . . . . . . . 5

1.31 -d Model for the Longitudinal Instability $\ldots \ldots \ldots \ldots$

1.3.1 Long Wavelength Approximation to the Cold Fluid Equations 7

1.3.2 Long Wavelength, Cold Beam Dispersion Relation . . . . . . 9

1.3.3 Methods for Controlling the Longitudinal Instability . . . . 10

1.4 Organization of the Thesis . . . . . . . . . . . . . . 11

2 The WARPrz Code 13

2.1 The WARPrz Code . . . . . . . . . . . . . . 13

2.2 Particle-in-Cell Method . . . . . . . . . . . . . . . . . 15

2.2 .1 Particle Advance . . . . . . . . . . . . . . 15

2.2.2 Interpolating the Particles to the Mesh . . . . . . . 17

2.2 .3 Field Solutions . . . . . . . . . . . . . . 19

2.2.4 Interpolating the Field to the Particles $\ldots \ldots \ldots \ldots 23$

2.3 Diagnostics . . . . . . . . . . . . . . . . . . 24

2.3.1 Line Charge Density . . . . . . . . . . . . . . 24 
2.3.2 Beam Current . . . . . . . . . . . . . . . 25

2.3.3 Particle Moments . . . . . . . . . . . . . . . 25

3 Model for the Module Impedance $\quad 26$

3.1 Model for the Resistive Wall in WARPrz . . . . . . . . . . . 26

3.2 Comparing the Model to 1-d Theory . . . . . . . . . . . . 29

3.3 Adding the Capacitive Component of the Module Impedance . . . . . 30

3.4 A Physical Picture for This Model . . . . . . . . . . . . . . . 31

3.4.1 Calculating the Radial Electric Field Between Irises . . . . . . 32

3.4.2 Calculating the Impedance of the Iris Structure $\ldots \ldots \ldots$

3.4 .3 Low Frequency Limit . . . . . . . . . . . . . . . . 39

3.4.4 Connecting the Iris Model to the WARPrz Wall Model . . . . 43

3.5 Dispersion Relation for Longitudinal Waves . . . . . . . . . . 45

3.5.1 Long Wavelength Limit. . . . . . . . . . . . . . . 47

3.6 Dispersion Relation Including Capacitive and Resistive Wall . . . . 47

3.6.1 Long Wavelength Limit. . . . . . . . . . . . . . . . 49

3.7 Testing This Model in WARPrz . . . . . . . . . . . . . 50

3.7.1 Particle Loading for Linear, Cold Beam Test Runs . . . . . . . 52

3.7.2 Results using Linear Particle Loading . . . . . . . . . . . 55

4 Simulations with Module Impedance $\quad 58$

4.1 Beam Temperature . . . . . . . . . . . . . . . . . 59

4.2 Simulations with a Purely Resistive Wall . . . . . . . . . 60

4.2.1 Large Resistance Simulation $(\eta=200 \Omega / \mathrm{m}) \ldots \ldots \ldots 60$

4.2.2 More Moderate, More Realistic Resistance Simulation ( $\eta=100$

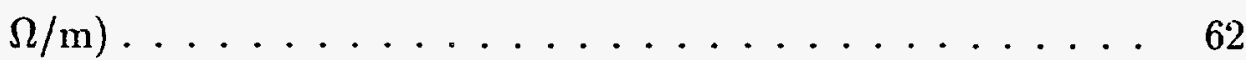

4.3 Simulations with Resistive and Capacitive Components . . . . . . 67

4.3 .1 Summary . . . . . . . . . . . . . . . 68

5 Effects of Temperature on Growth Rate $\quad 70$

5.1 Including Longitudinal Temperature $\ldots \ldots \ldots \ldots \ldots$ 
5.2 A Coupling Mechanism for Perpendicular Temperature and Wave Damp-

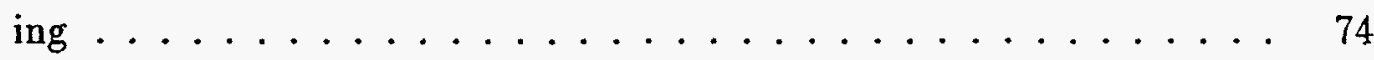

5.3 A Code Using the Simple Model . . . . . . . . . . . . . . . 77

5.4 Growth Rates with Finite Temperature from WARPrz $\ldots \ldots \ldots$

6 Intermittently-Applied Axial Confining Fields 91

6.1 1-d Fluid Model . . . . . . . . . . . . . . . . . . . . . . 92

6.2 Particle Simulations . . . . . . . . . . . . . . . . . 96

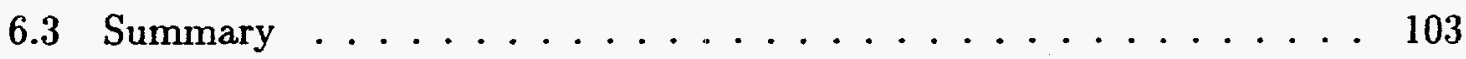

7 Conclusions and Future Work 106

7.1 Conclusions . . . . . . . . . . . . . . 106

7.2 Future Work . . . . . . . . . . . . . . . 108

$\begin{array}{ll}\text { A List of Symbols } & 110\end{array}$

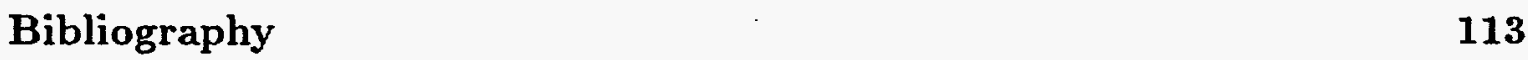




\section{List of Figures}

1.1 A cartoon of a heavy ion fusion driver showing the particle energy, beam current, and pulse duration at several locations along the device.

2.1 Area weighting is used to determine the fraction of each particle's charge that goes to the four surrounding gridpoints $\ldots \ldots \ldots 17$

2.2 The grid used for the field solution. The dashed line indicates the volume used to calculate the density from the charge. . . . . . . . . .

3.1 The advection is just a remapping of the values to the correct array index if the mesh moves by an integer number of grid cells per timestep 29

3.2 Side view of iris structure. Structure is cylindrically symmetric about center axis . . . . . . . . . . . . . . . 32

3.3 End view of iris structure $\ldots \ldots \ldots \ldots \ldots \ldots \ldots$

3.4 Side view close up of a single pair of irises and their dimensions . . . 34

3.5 The radial electric field (as a fraction of its value at the inner radius) as a function of $x=n \pi r / h$ for irises 5 times deeper than long. The dashed, vertical line shows the position of the inner radius at $x_{0}=5 \pi$.

3.6 The radial electric field (as a fraction of its value at the inner radius) as a function of $x=n \pi r / h$ for irises half as deep as long. The dashed, vertical line shows the position of the inner radius at $x_{0}=5 \pi \ldots \ldots$

3.7 The radial electric field (as a fraction of its value at the inner radius) as a function of $x=n \pi r / h$ for irises 45 times deeper than long. The dashed, vertical line shows the position of the inner radius at $x_{0}=5 \pi$ 
3.8 The real part of the impedance approaches $100 \mathrm{Ohms} / \mathrm{m}$ as $\mathrm{x}$ (proportional to frequency) gets small . . . . . . . . . . . . . . . 39

3.9 The imaginary part of the impedance becomes linear as $\mathrm{x}$ (proportional to frequency) gets small . . . . . . . . . . . . . . . . . . . 40

3.10 A plot of $\left|(k b)^{2} / 2 \ln (f k b / 2)\right|$ as a function of $k b$ and the fraction of the pipe which is filled, $f$, shows that this quantity is smaller than 1 for $k b$ less than $2 \ldots \ldots \ldots \ldots \ldots \ldots$

4.1 A simulation with $200 \Omega / \mathrm{m}$ resistance shows large amounts of growth. (a) $6.6 \mu \mathrm{s}$, (b) $10.9 \mu \mathrm{s}$, (c) $17.5 \mu \mathrm{s} \ldots \ldots \ldots$

4.2 A simulation with $100 \Omega / \mathrm{m}$ resistance shows moderate growth. (a) 6.6 $\mu \mathrm{s}$, (b) $10.9 \mu \mathrm{s}$, (c) $17.5 \mu \mathrm{s} \ldots \ldots \ldots \ldots$

4.3 The perturbation reflects off the beam end and decays as it travels forward. (a) $28.4 \mu \mathrm{s}$, (b) $35.0 \mu \mathrm{s}$, (c) $39.4 \mu \mathrm{s} \ldots \ldots \ldots 5$

4.4 Real part of the frequency as a function of wavenumber . . . . . . . 66

4.5 Growth rate of the instability vs wavelength for 3 values of the wall

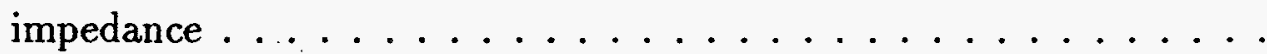

4.6 When capacitance is added to the system, a larger perturbation is launched, but little growth occurs (a) $6.6 \mu \mathrm{s}$, (b) $10.9 \mu \mathrm{s}$, (c) $17.5 \mu \mathrm{s}$.

5.1 Growth rate as a function of longitudinal thermal speed. The vertical line represents the largest thermal speed that can be focused to the necessary spot size. . . . . . . . . . . . . . .

5.2 The simple model approximates the surface wave as an oscillating wall which particles reflect off.

5.3 Resonances are seen for particles which reflect off the oscillating wall every $1,2,3$, and 4 oscillations. Vertical lines indicate resonant velocities. 78 
5.4 Sum of the square of the particle velocities (proportional to the total energy) increases with time as the particles gain energy from the oscillating wall. The increase in energy levels off as the distribution function is flattened in the region surrounding the resonance. The oscillations are at the wall oscillation frequency. . . . . . . . . . . . .

5.5 The distribution of particles as a function of velocity at the beginning of the run (a) and the end of the run (b) shows the distribution flattens out at the $n=1$ resonance $\ldots \ldots \ldots \ldots \ldots \ldots$

5.6 The slope of the straight line is equal to twice the growth rate . . . 83

5.7 No growth is seen in case (c) . . . . . . . . . . . . . . 85

5.8 Although the wave is not growing, the increase in perpendicular particle kinetic energy (Joules) is less than $1 \%$ over more than 7 microseconds. 86

5.9 When no resistive wall is present, we expect the wave to decay. Here we see the field energy decrease when a $4 \%$ forward and a $4 \%$ backward wave are excited. . . . . . . . . . . . . . .

5.10 Although the field energy is decreasing, the increase in perpendicular kinetic energy is not large enough to compensate. . . . . . . .

5.11 The energy of the wave is split between the field energy and the $Z$ kinetic energy.

5.12 The total energy of the system is conserved to within $.1 \%$ but the amount of energy lost by the field energy is also about $.1 \% \ldots \ldots$.

6.1 Fluid velocity (scaled to $v_{p}$ ) as a function of axial position after 10 applications of the ear fields . . . . . . . . . . .

6.2 Line charge density as a function of axial position after 10 applications

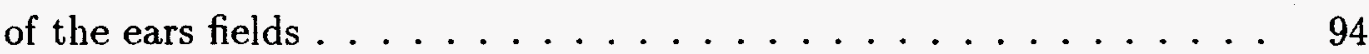

6.3 Position of grid points near the beam head shows the expansion and contraction of the beam. . . . . . . . . . . . . . 
6.4 One cycle of intermittently-applied ears. (a) Initial phase space (b) Beam expands (c) Ear Field is applied (d) Beam is compressed (e) Beam expands back to its initial state . . . . . . . . . . 98

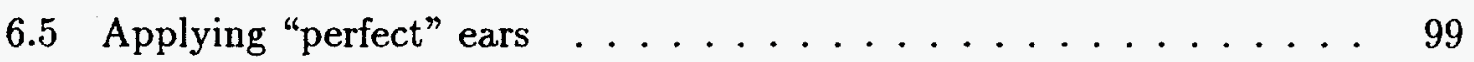

6.6 Applying ears every 200 meters excites a train of perturbations on the beam. . . . . . . . . . . . . . . . . . . 100

6.7 Ear field and error introduced . . . . . . . . . . . . . 101

6.8 Applying ears that are always too large . . . . . . . . . . . 101

6.9 Applying ears that alternate too large and too small . . . . . . . 102

6.10 An error of random amplitude was introduced on each application by multiplying the error ear field by the random amplitude . . . . . . 104

6.11 A random error in the ears excites the most unstable wavelength of the longitudinal instability . . . . . . . . . . . 105 
Debra Ann Callahan Miller March 1994

APPLIED SCIENCE

\author{
Simulations of Longitudinal Beam Dynamics \\ of Space-Charge Dominated Beams for Heavy Ion Fusion
}

\begin{abstract}
The longitudinal instability has potentially disastrous effects on the ion beams used for heavy ion driven inertial confinement fusion. This instability is a "resistive wall" instability with the impedance coming from the induction modules in the accelerator used as a driver. This instability can greatly amplify perturbations launched from the beam head and can prevent focusing of the beam onto the small spot necessary for fusion.

This instability has been studied using the WARPrz particle-in-cell code. WARPrz is a $21 / 2$ dimensional electrostatic axisymmetric code. This code includes a model for the impedance of the induction modules.

Simulations with resistances similar to that expected in a driver show moderate amounts of growth from the instability as a perturbation travels from beam head to tail as predicted by cold beam fluid theory. The perturbation reflects off the beam tail and decays as it travels toward the beam head. Nonlinear effects cause the perturbation to steepen during reflection.

Including the capacitive component of the module impedance has a partially stabilizing effect on the longitudinal instability. This reduction in the growth rate is seen in both cold beam fluid theory and in simulations with WARPrz.

Instability growth rates for warm beams measured from WARPrz are lower than cold beam fluid theory predicts. Longitudinal thermal spread cannot account for this
\end{abstract}


decrease in the growth rate. A mechanism for coupling the transverse thermal spread to decay of the longitudinal waves is presented.

The intermittency of the axial confining fields ("ear" fields) is expected to be a major source of beam perturbations. Simulations with intermittently-applied "ear" fields have shown that, if applied carefully, these fields do not generate large perturbations. Errors in these fields can, however, excite the longitudinal instability.

The longitudinal instability is no longer a threat to the heavy ion fusion program. The simulations in this thesis have shown that the growth rate for this instability will not be as large as earlier calculations predicted. These studies have shown that including the capacitive component of the module impedance, which was neglected in early calculations, greatly reduces the growth rate of the instability. In addition, a transverse thermal spread on the beam can reduce, and in some cases stabilize, this instability. 


\section{Chapter 1}

\section{Introduction}

\subsection{Heavy Ion Fusion}

Heavy ion beam driven inertial fusion is an attractive candidate for inertial fusion energy production because of the high efficiency of the accelerators used as drivers[11]. The US Heavy Ion Fusion program is based on a linear induction accelerator, with cost savings through a recirculating induction accelerator also being pursued[2, 29].

Figure (1.1) shows a cartoon of a heavy ion fusion (HIF) driver. Ions of mass about $200 \mathrm{amu}$ are injected at $10 \mathrm{MeV}$ with a current of 15 Amps. The ions then go into an induction accelerator with electrostatic focusing. An induction accelerator can be thought of as a 1-turn transformer with the beam acting as the secondary. The beam has a large current density and so the space charge forces are large (spacecharge-dominated) and focusing is important.

The beam is focused with an alternating gradient (AG) focusing structure. In AG focusing, quadrupole fields are used for focusing. One set of quadrupoles focus the beam in the $x$-direction and defocus the beam in the $y$-direction. On the next element, the focusing/defocusing is reversed. There is a net focusing because the field is larger close to the quadrupole focusing elements. Thus, the beam is focused more than defocused because the beam is farther from the elements when it is defocused.

In the low energy end of the accelerator, electrostatic AG focusing can be used. In 


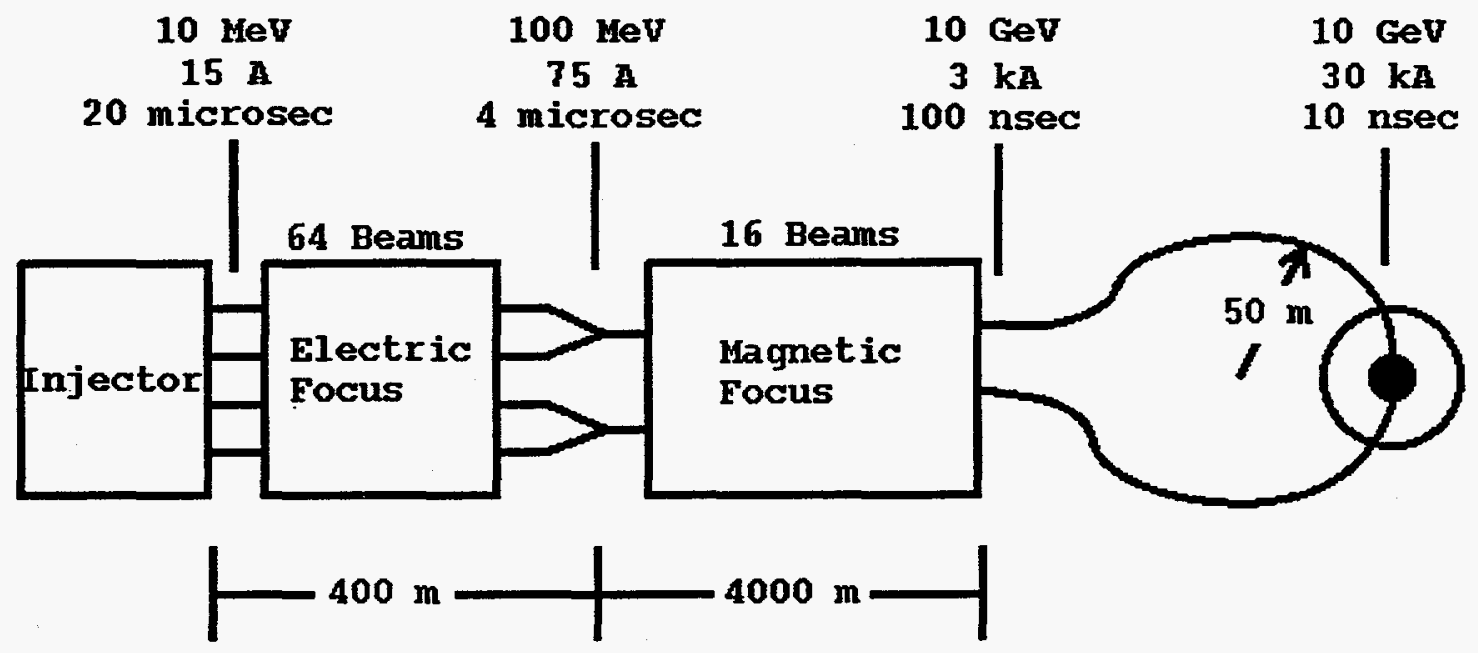

Figure 1.1: A cartoon of a heavy ion fusion driver showing the particle energy, beam current, and pulse duration at several locations along the device.

the higher energy sections, magnetic $\mathrm{AG}$ focusing is needed because the force on the beam due to magnetic fields increases along with the beam speed via the Lorentz force law, $\mathbf{F}_{\text {magnetic }}=q \mathbf{v} \times \mathbf{B}$ whereas the force due to the electric field remains constant. As the beam speed increases the time spent in the region with the focusing fields decreases and thus the particle velocities are less affected when electric focusing is applied. For magnetic focusing, the time spend in the focusing region decreases, but the force increases as the beam speed increases. Thus, we must use magnetic focusing in the high energy parts of the accelerator.

In the accelerator section, the current is carried in 16-64 beams. The current is divided up and the beams shielded from one another so that the space charge forces are not so large. The MBE-4 experiment at Lawrence Berkeley Laboratory demonstrated the ability to accelerate, focus, and handle the current spread over four beams $[1,9]$. 
Each beam is accelerated from $10 \mathrm{MeV}$ to $10 \mathrm{GeV}$. A longitudinal velocity tilt is added to the beam so the beam tail is moving faster than the beam head. This makes the beam compress as it drifts. The beam is compressed by a factor of 10 in length giving it a pulse duration of $10 \mathrm{nsec}$ and current of $30 \mathrm{kA}$. As the beams drift compress, they are curved around and focused with magnetic lenses onto a two sided ICF target.

Most of the physics issues for HIF revolve around manipulating the ion beam while keeping the beam's emittance (proportional to the beam's phase space volume) small. The emittance must remain small so that the necessary power density is delivered to the target. In the longitudinal direction, chromatic aberration in the magnetic lens system causes particles with different longitudinal velocities to be focused at different locations and thus keeping the longitudinal momentum spread small is also important.

\subsection{The Longitudinal Wall Impedance Instability}

The longitudinal wall impedance instability is a "resistive wall" instability. The physics is the same as in resistive wall amplifiers[3], which have been around for 40 years. In an HIF driver, the impedance which drives the instability is the impedance of induction accelerating modules. The instability is set up because a perturbation in the beam current produces a corresponding perturbation in the return current which flows through the wall. Ohm's law tells us that the return current flowing through a wall resistance (or more generally, an impedance) requires an electric field. This electric field acting back on the beam can enhance the perturbation in the beam current and thus an unstable situation develops. One dimensional cold beam, fluid theory (presented in section 1.3) predicts two waves-a wave which travels forward in the beam frame that decays exponentially in time and a wave which travels backward in the beam frame that grows exponentially in time.

This instability has potentially disastrous effects on beams used in a heavy ion fusion driver because it can cause small perturbations launched from the beam head 
(from errors in the accelerating fields for example) to be amplified as they travel the length of the beam. These large perturbations can cause the spread in momentum to become too large. It is generally believed that a momentum spread of $\Delta p / p \leq 1 \%$ is acceptable at the focusing system. If the beam is drift compressed by a factor of 10 , we are allowed a maximum momentum spread of $\Delta p / p=0.1 \%$ at the end of the accelerator. Early calculations predicted tens of $e$-foldings of growth due to the instability[20] which caused much concern for the the heavy ion fusion program.

Due to the long growth times, the longitudinal instability will not be seen in nearterm HIF experiments which are small in size. This makes a complete study of this instability important for the HIF program.

An experimental effort to study longitudinal beam dynamics in space charge dominated electron beams is underway at the University of Maryland under the direction of Martin Reiser[27, 24, 7, 28, 25]. These experiments are being modeled with WARPrz[13]. The UM group plans to study the longitudinal instability by passing the electron beam through a tube with a high resistance coating. The large resistance, small mass, and scaling of other parameters increases the growth rate by two orders of magnitude over an HIF driver[22]. Even so, the resistive tube used in this experiment is only 1 meter long and so theory predicts increases in wave amplitude of only about $25 \%$ in this experiment.

To do a complete study of this instability, we have written the WARPrz particlein-cell code. This code is part of the WARP family of codes written specifically to model HIF driver physics quickly and efficiently. The code will be described fully in chapter 2. With this code, we have simulated this instability over distances of several kilometers, have seen wave reflection off the beam bunch ends, and have seen the partially stabilizing effect of the capacitive component of the module impedance. We have studied the effects of beam temperature on this instability. In addition, we have studied intermittently applied axial confining fields as a source of perturbations for this instability.

These studies have shown that the longitudinal instability is not as serious a 
problem for HIF as was once feared. The simulations in this thesis include physics neglected in the early calculations which reduced the growth rate of the instability considerably. This reduction in the growth rate, coupled with an understanding of perturbations generated on the beam, has reduced the threat to HIF from the longitudinal instability.

\subsection{1-d Model for the Longitudinal Instability}

Most of the analytical work done on this instability has used a one dimensional, cold fluid model in the long wavelength limit. This instability is worst when the wavelength is long compared with the beam radius. Although the purpose of this thesis is to explore two dimensional and thermal effects, the 1-d, cold fluid theory is an appropriate starting point.

\subsubsection{Long Wavelength Approximation to the Cold Fluid Equations}

The longitudinal dynamics of the ion beam can be approximately described using a 1-d cold fluid model. The description is done in terms of the fluid velocity, $u$ and the line charge density, $\lambda$. The line charge density, which has units of charge per length, is defined to be

$$
\lambda(z)=\int_{0}^{2 \pi} \int_{0}^{a} \rho(r, \theta, z) r d r d \theta
$$

where $\rho$ is the charge density and $a$ is the beam radius. If we assume an incompressible fluid, then the line charge density can be written as

$$
\lambda(z)=\rho \pi a^{2}(z)
$$

We write a continuity equation for the line charge density

$$
\frac{\partial \lambda}{\partial t}+\frac{\partial(\lambda u)}{\partial z}=0
$$


The conservation of momentum equation is written in the usual way:

$$
\frac{\partial u}{\partial t}+u \frac{\partial u}{\partial z}=\frac{Z e}{M} E_{z}
$$

where $M$ is the ion mass and $Z e$ is the charge.

Here $E_{z}$ includes the beam's self field plus any external fields. We need to describe the $z$ component of the self electric field in terms of the line charge density and the fluid velocity. A physically motivated derivation of self electric field can be obtained by doing the following. Imagine a beam of charge with constant radius, $a$, in a conducting pipe of radius $r_{\text {wall }}$. From Gauss' law, the radial electric field is given by

$$
E_{r}(r)= \begin{cases}\frac{\rho r}{2 \epsilon_{0}}, & \text { if } r \leq a, \\ \frac{\rho a^{2}}{2 \epsilon_{0} r}, & \text { if } a \leq r \leq r_{\text {wall }} .\end{cases}
$$

We calculate an electrostatic potential from $E_{\tau}=-\partial \phi / \partial r$ and require that $\phi$ be continuous at $r=a$ and $\phi=0$ at $r=r_{\text {wall }}$ (perfectly conducting wall). This results in the electrostatic potential:

$$
\phi(r)= \begin{cases}\frac{\rho}{4 \epsilon_{0}}\left(a^{2}-r^{2}\right)+\frac{\rho a^{2}}{2 \epsilon_{0}} \ln \left(r_{\text {wall }} / a\right), & \text { if } r \leq a, \\ \frac{\rho a^{2}}{2 \epsilon_{0}} \ln \left(r_{\text {wall }} / r\right), & \text { if } a \leq r \leq r_{\text {wall }} .\end{cases}
$$

Let the beam radius vary slowly with $z$. If the wavelength of this variation is long compared with the beam radius, we can get the $z$ component of the electric field by using the chain rule.

$$
E_{z}(z)=-\frac{\partial \phi}{\partial a} \frac{\partial a}{\partial z}=-\frac{\rho a}{\epsilon_{0}} \frac{\partial a}{\partial z} \ln \left(r_{\text {wall }} / a\right)
$$

inside the beam. For an incompressible beam, use equation (1.2)

$$
\frac{1}{2 \pi} \frac{\partial \lambda}{\partial z}=\rho a \frac{\partial a}{\partial z}
$$

This leaves us with an expression for the $z$ component of the self electric field in terms of the line charge density,

$$
E_{z}=-\frac{\ln \left(r_{\text {wall }} / a\right)}{2 \pi \epsilon_{0}} \frac{\partial \lambda}{\partial z}=-g \frac{\partial \lambda}{\partial z}
$$


where we define the "g-factor,"

$$
g=\frac{\ln \left(r_{\text {wall }} / a\right)}{2 \pi \epsilon_{0}}
$$

which depends on the geometry and the system of units (MKS).

The $z$ component of the self field is constant across the beam radius when the density is constant. In the beam frame, the fields are electrostatic and so,

$$
\nabla \times \mathbf{E}=0
$$

For a cylindrically symmetric system, this reduces to

$$
\frac{\partial E_{r}}{\partial z}-\frac{\partial E_{z}}{\partial r}=0
$$

The radial self field (equation (1.5)) is independent of $z$ inside the beam $(r \leq a)$, so

$$
\frac{\partial E_{\tau}}{\partial z}=0
$$

and equation (1.12) then becomes

$$
\frac{\partial E_{z}}{\partial r}=0
$$

This tells us the $z$ component of the self electric field is independent of $r$.

\subsubsection{Long Wavelength, Cold Beam Dispersion Relation}

We can calculate a dispersion relation for a beam in a pipe with a resistive wall using the fluid equations (1.3), (1.4). Linearize these equations with $u=\tilde{u}$ in the beam frame, and $\lambda=\lambda_{0}+\tilde{\lambda}$ where $\tilde{u}$ and $\tilde{\lambda}$ are small quantities. The perturbed electric field consists of the self field from equation (1.9) plus the electric field due to the resistive wall.

$$
\tilde{E}_{z}=-g \frac{\partial \tilde{\lambda}}{\partial z}-\eta \delta I_{b}
$$

where $\eta$ is the resistance per unit length. The perturbed beam current is given by $\delta I_{b}=v_{b} \tilde{\lambda}+\lambda_{0} \tilde{u}$, where $v_{b}$ is the beam velocity. If we let. $\tilde{\lambda}, \tilde{u} \sim e^{i(k z-\omega t)}$, and use equations (1.3), (1.4), and (1.15), we find the dispersion relation in the beam frame,

$$
0=1-\frac{k^{2} v_{p}^{2}}{\omega^{2}}+i \frac{\eta v_{p}^{2}}{g} \frac{\left(k v_{b}+\omega\right)}{\omega^{2}}
$$


where the wave phase velocity is

$$
v_{p}^{2}=\frac{Z e g \lambda_{0}}{M} .
$$

If we assume $v_{b}>>\omega / k$ and $\eta v_{b} / g k<<1$, then the dispersion relation can be rewritten as

$$
\frac{\omega}{k v_{p}}= \pm \sqrt{1-i \frac{\eta v_{b}}{g k}} \approx \pm\left(1-i \frac{\eta v_{b}}{2 g k}\right)
$$

The real part of this equation gives a wave traveling with the phase velocity, $\omega_{\text {real }} / k=$ $\pm v_{p}$. The forward traveling wave $\left(\omega_{\text {real }}=+k v_{p}\right)$ damps with decay rate $\omega_{\mathrm{i}}=$ $-\eta v_{b} v_{p} / 2 g$, while the backward traveling wave $\left(\omega_{\text {real }}=-k v_{p}\right)$ grows with growth rate $\omega_{\mathrm{i}}=+\eta v_{b} v_{p} / 2 g$. For long wavelength perturbations, we expect to see forward traveling waves damp and backward traveling waves grow independent of wavelength.

\subsubsection{Methods for Controlling the Longitudinal Instability}

The derivation presented in the previous section is a worst case for this instability. Since forward traveling waves damp, it is possible for a backward traveling wave to reflect off the beam end, become a forward traveling wave, and decay. Others have found that reflections off the beam end can limit the amount of growth if the perturbation undergoes a small number $(\sim 1) e$-folding in the time it takes to travel from the beam head to tail[ $[16,5]$. We see a similar effect in chapter 4 when we vary the size of the wall resistance.

Longitudinal temperature can also stabilize this mode. The limit on $\Delta p / p \leq .1 \%$ at the end of the accelerator puts a restriction on the longitudinal temperature,

$$
T_{z}=\frac{\Delta p^{2}}{2 M}=\left(\frac{\Delta p}{p}\right)^{2} \frac{p^{2}}{2 M}
$$

At the end of the accelerator, the maximum longitudinal temperature is given when $\Delta p / p=.001$ and the beam energy $p^{2} / 2 M=10 \mathrm{GeV}$ This gives a maximum longitudinal temperature of

$$
T_{z}=10^{-6} 10^{10} \mathrm{eV}=10 \mathrm{keV}
$$


In chapter 5, we will follow the derivation due to Reiser which shows that given a maximum longitudinal temperature of $T_{z}=10 \mathrm{keV}$, longitudinal temperature has little or no effect on the growth rate.

Because the beams for HIF travel at a small fraction of the speed of light $\left(v_{b} \leq\right.$ $c / 3$ ), it is possible to use "feed-forward" stabilization. With this technique, perturbations on the beam are detected at one point along the accelerator, a signal is sent ahead, and a correcting field is applied downstream. Hahn has developed an algorithm for "feed-forward" stabilization and has suppressed growth from the longitudinal instability[14].

\subsection{Organization of the Thesis}

This thesis discusses several aspects of longitudinal dynamics of beams needed for HIF.

Chapter 2 discusses the WARPrz code which was used to simulate the physics in the rest of the thesis.

Chapter 3 describes the model for the module impedance used in WARPrz. A physical equivalent of this model is discussed. The dispersion relation for the longitudinal instability including both resistive and capacitive components of the impedance is derived from a $2-\mathrm{d}$ cold beam fluid theory. Tests of the model in WARPrz when WARPrz is run with a cold beam are compared with the cold beam fluid theory.

Chapter 4 describes simulations of the longitudinal instability in finite length, warm beams. Simulations are done with and without a capacitive component of the module impedance. In each case, a wave is launched from the beam head and grows as it travels the length of the beam. Reflection of the perturbation off the beam tail and non-linear effects are seen. Growth rates are measured to be smaller than cold beam theory predicts due to finite temperature.

Chapter 5 discusses the effect of temperature on the growth rate of the longitudinal instability. Longitudinal temperature is shown to have little effect on the growth rate 
in the long wavelength limit. A mechanism for coupling perpendicular temperature and wave damping is proposed. The effect of temperature on the growth rate is explored with WARPrz.

Chapter 6 describes simulations including intermittently-applied axial confining fields. These fields can excite perturbations on the beam that are amplified by the longitudinal instability. The effect of errors in the confining fields is shown.

Chapter 7 contains conclusions and future directions.

The main purpose of this thesis was to study the effects of the longitudinal instability on a heavy ion fusion driver. If early predictions of 10's of $e$-foldings of growth were correct, this instability could have ended the heavy ion fusion program. Instead, this instability is no longer considered a major problem for HIF, in part due to the simulations presented here. These simulations show that the capacitive component of the module impedance and transverse thermal spread in the beam reduce the growth rate considerably from the value predicted by the early calculations. In addition, simulations of the intermittently applied axial confining fields (thought to be a major source of "seed" perturbations for the instability) show that these fields can be applied without creating large beam perturbations. The major conclusion of this thesis is that the longitudinal instability is no longer a "show stopper" for the heavy ion fusion program. 


\section{Chapter 2}

\section{The WARPrz Code}

\subsection{The WARPrz Code}

WARP is a family of codes begun by Alex Friedman and David Grote specifically for studying heavy ion fusion driver issues. The code currently consists of five physics packages-WARP3d, a 3 dimensional (6 phase space variables) Cartesian coordinate particle in cell code; WARPrz, a $21 / 2$ dimensional, cylindrically symmetric particle in cell code; F3d, the 3-d, electrostatic field solver; Frz, the RZ, electrostatic field solver; and Envelope, an envelope equation solver. The code is built upon the BASIS code development system which allows us to use dynamically allocated arrays and an interpreter from which we can make changes in variables, call subroutines, and make plots at any time during the simulation. Thanks to work done by David Grote, we are able to use the NCAR graphics package as well as the GRAFLIB graphics package. The code has run on a CRAY YMP (at Livermore's Secure Computing Facility), CRAY 2's and the C-90 (at NERSC), IBM RS6000's (at Livermore's Open Computing Facility) and on SUN workstations.

WARPrz is a $21 / 2$ dimensional particle in cell code. The particles exist in a cylindrical geometry and it is assumed that there are no variations in the azimuthal (theta) direction. The particles have two coordinate positions ( $r$ and $z)$ and three components of velocity $\left(v_{r}, v_{\theta}\right.$ and $\left.v_{z}\right)$. Since the particles are all moving with velocity 
$v_{\text {beam }}$ down the $z$ axis, we use a mesh moving with the beam for the field solves. In this frame, the magnetic field is down from the electric field by a factor of $(u / c)^{2}$ where $u$ is the axial particle velocity in the beam frame. In the beam frame, particles move very slowly compared with the speed of light, and so self fields are electrostatic in that frame. Due to the modular structure of a BASIS code, we could replace the electrostatic fields by electromagnetic fields by adding another field solve package if that were necessary.

Since our beam contains ions without any electrons, we must model the external confinement fields in order to keep the beam from breaking up. In experiments, the radial confinement is done through an alternating gradient focusing system. This AG focusing is inherently three dimensional and so we must mock it up in the axisymmetric code. This is done by simply adding an external, radial electric field that is linear in $r$. This field is calculated at the beginning of the run and then used throughout the simulation. Three options are available for specifying this external field-the slope of the radial field can be specified, the radial field can be gotten from the charge density and Gauss' law, or a linear least squares fit can be done to the radial self-field and then a field with the opposite sign is applied.

The confinement in the axial direction is done through "ears" fields. The ears are external axial electric fields which are applied at the ends of the beam to keep it confined (hence the name). In the experiment, these fields will be applied at certain laboratory positions as the beam travels down the accelerator (e.g. every 100 meters). This means that the beam is continually expanding and contracting as it passes the ears fields. WARPrz generally uses continuously applied ear fields. These fields are applied at every time step and keep the beam from expanding or contracting. These ear fields are composed of two pieces: an electric field to compensate for the space charge effects and a smaller electric field to compensate for the pressure[21]. Since there is little change in the electric field due to space charge at different radii, this part of the ears fields is gotten from the initial electric field on the beam axis:

We have also done some simulations of intermittently applied ears (which would 
be closer to the experimental set up). These simulations will be discussed in detail in chapter 6 .

\subsection{Particle-in-Cell Method}

Particle-in-cell (PIC) codes are heavily used in both plasma physics and accelerator physics. For an electrostatic PIC code, the computational cycle is as follows. Particle velocities are updated using the Lorentz force. Once the new velocity is found, the particle position is updated. The charge density is calculated on a mesh by assigning a fraction each particle's charge to nearby gridpoints. Once the charge density has been calculated, the electrostatic potential is found on the grid from the Poisson equation and the components of the electric fields are calculated at each gridpoint. The fields are then interpolated to the position of each particle and the cycle continues.

For an electromagnetic code, both the charge density and the current density are gathered from the particles on the mesh. Maxwell's equations (or an approximation to Maxwell's equations, such as the Darwin model) are then solved instead of the simple Poisson equation.

WARPrz is an electrostatic PIC code with the addition of the resistive wall boundary condition used in modeling the longitudinal instability, The scheme is based heavily on the one presented by Birdsall and Langdon[4].

\subsubsection{Particle Advance}

Velocity Advance

The particles are moved by integrating the equations of motion

$$
\begin{gathered}
m \frac{d \mathbf{v}}{d t}=q(\mathbf{E}+\mathbf{v} \times \mathbf{B}) \\
\frac{d \mathbf{x}}{d t}=\mathbf{v}
\end{gathered}
$$


Since we want a centered difference scheme with $\mathrm{x}$ known at integer time steps, we need to calculate $\mathbf{v}$ at half time steps. We write a centered difference approximation to equation (2.1)

$$
\frac{\mathbf{v}^{n+1 / 2}-\mathbf{v}^{n-1 / 2}}{\Delta t}=\frac{q}{m}\left(E^{n}+\frac{\mathbf{v}^{n+1 / 2}+\mathbf{v}^{n-1 / 2}}{2} \times B^{\mathbf{n}}\right)
$$

The velocities are updated using the well-known Boris method which is described in Birdsall and Langdon[4] sections 4-3 and 4-4. The Boris scheme separates the electric and magnetic forces completely. The scheme breaks down into three steps: add half the electric impulse to the velocity, apply a rotation which applies the magnetic force, then add the other half of the electric impulse.

Having the particle velocities at half time steps gives a scheme which is second order accurate in $\Delta t$, but it is inconvenient for doing diagnostics since the fields and positions are known at integer time steps. To circumvent this problem, WARPrz has three types of particle velocity advance. First is the "fullv" advance which advance the velocities from time $n-1 / 2$ to time $n+1 / 2$ as described above. This is the advance done for most of the run. Just before diagnostics are done a "synchv" (synchronize velocity) step is done to synchronize the velocity and position. In a sychronizing step, we apply half the electric field contribution to the velocity advance and then rotate half way. At the beginning of the run and just after diagnostics have been done, we need to advance the velocity by one half a step and so a "halfv" step is done by doing half the rotation followed by half the electric field contribution.

\section{Position Advance}

In cylindrical coordinates, the origin can be a problem. To avoid this, we do the particle advance in Cartesian coordinates as is done in Birdsall and Langdon[4] section 14-12. If we have a particle at position $r_{1}, z_{1}$ at time $n$ and its velocities $v_{r, 1}, v_{\theta, 1}$, $v_{z, 1}$ at time $n+1 / 2$, then we update the positions by

$$
\begin{gathered}
x_{2}^{\prime}=r_{1}+v_{r, 1} \Delta t \\
y_{2}^{\prime}=v_{\theta, 1} \Delta t
\end{gathered}
$$


then at time $n+1$ we have $r_{2}$

$$
r_{2}=\sqrt{\left(x_{2}^{\prime}\right)^{2}+\left(y_{2}^{\prime}\right)^{2}}
$$

We also have to rotate the coordinate system because the velocities $v_{r}$ and $v_{\theta}$ all refered to the coordinate system used before we updated the particle positions.

$$
\left(\begin{array}{l}
v_{r} \\
v_{\theta}
\end{array}\right)_{2}=\left(\begin{array}{cc}
\cos \alpha & \sin \alpha \\
-\sin \alpha & \cos \alpha
\end{array}\right)\left(\begin{array}{l}
v_{r} \\
v_{\theta}
\end{array}\right)_{1}
$$

where $\sin \alpha=y_{2}^{\prime} / r_{2}$ and $\cos \alpha=x_{2}^{\prime} / r_{2}$. If a particle stops at $r_{2}=0$, the code sets $\cos \alpha=1$ and $\sin \alpha=0$ and all the particle's momentum is in the radial direction. This rotation is possible because the system is azimuthally symmetric.

\subsubsection{Interpolating the Particles to the Mesh}

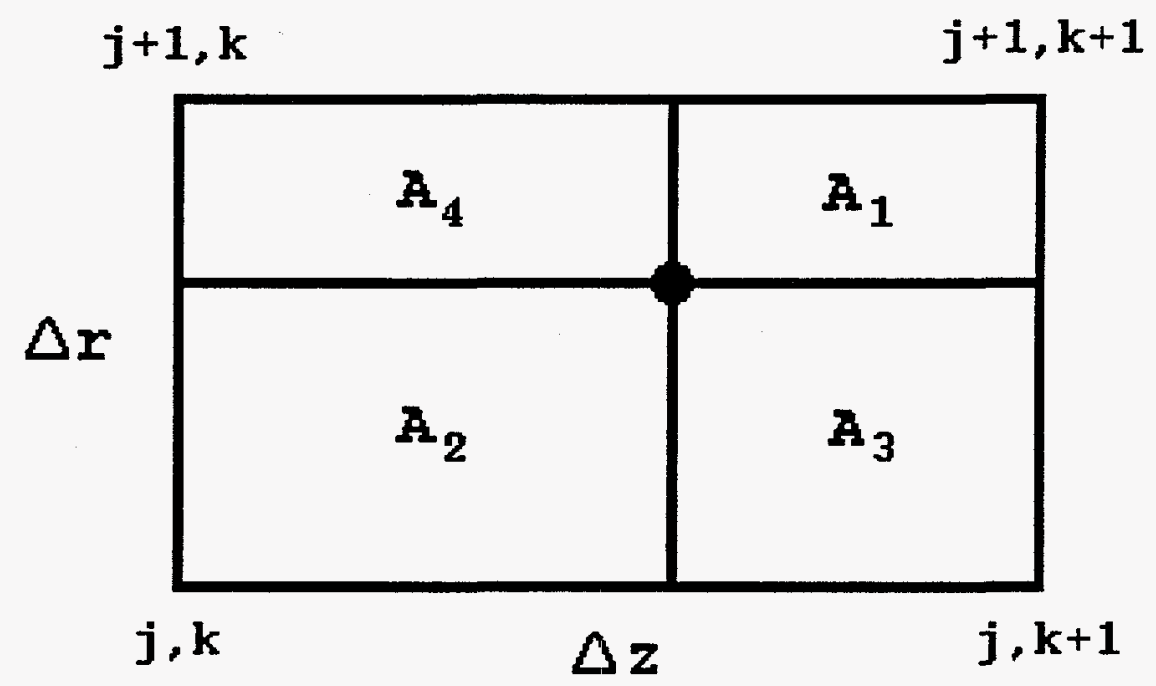

Figure 2.1: Area weighting is used to determine the fraction of each particle's charge that goes to the four surrounding gridpoints 
An area weighting scheme is used to interpolate the charge of each particle to the four surrounding gridpoints. Figure (2.1) shows the areas used in the interpolation scheme. The contribution of the particle shown in figure $(2.1)$ to gridpoint $(j, k)$ is calculated by

$$
Q_{j, k}=q w A_{1} /(\Delta r \Delta z)
$$

where $w$ is the number of real particles that each simulation particle represents. the charge density is calculated from the charge by

$$
\rho_{j, k}=Q_{j, k} / V_{j, k}
$$

For $j \neq 0$, the volume is

$$
V_{j, k}=\pi \Delta z\left(r_{j+1 / 2}^{2}-r_{j-1 / 2}^{2}\right)
$$

where $r_{j+1 / 2}$ is the radius half way between the gridpoints, $r_{j+1 / 2}=\frac{1}{2}\left(r_{j+1}+r_{j}\right)$. For a constant grid spacing, $\Delta r$, this simplifies to

$$
V_{j, k}=2 \pi r_{j} \Delta r \Delta z
$$

For $j=0$, the volume is

$$
V_{0, k}=\pi \Delta z r_{1 / 2}^{2}=\frac{\pi}{4}(\Delta r)^{2} \Delta z
$$

The interpolation of the charge to the grid is the most time consuming parts of the WARPrz code because this step does not vectorize well. We can vectorize the deposition of one particle's charge to the surrounding gridpoints. In WARPrz, this results in a vector length of only 4 . In the 3 -d code, this gives a vector length of 8 , since each particle has 8 nearest neighbors.

Horowitz[17] used a technique for vectorizing the particle deposition that involved sorting the particles into groups so that no two particles in a group contributed to the same gridpoint. The cost of such a technique is the cost of sorting the particles. In Horowitz' code, which was a 3-d electromagnetic PIC code, the cost of sorting the particles was smaller than the gain he got from the vectorized deposition and so it was worth it to sort the particles. 
We tried a sorting similar to that of Horowitz with WARPrz. The time spent sorting was about the same as Horowitz found. However, the gain from the vectorized deposition was about the same as the sorting time. So the total time to deposit the charge, including the sort, was about the same as it was when we vectorized over vector length 4 . This is a difference between an electrostatic and an electromagnetic PIC code. In the electrostatic code, we only deposit the charge density while in an electromagnetic code, we also deposit three components of current density. In the electromagnetic code, one sort is offset by vectorizing the deposition of four quantities (charge density and three current density components). In the electrostatic code, for the price of one sort, we only get to vectorize the deposition of one quantity. Because we didn't get any gain in speed by sorting the particles, we went back to the original deposition and vectorized over a length of 4 for each particle.

\subsubsection{Field Solutions}

The field solutions are done in a window moving with the beam. At present, the beams in WARPrz do not accelerate, so this window moves with a constant beam velocity, $v_{\text {bean }}$. In this moving frame, the forces due to self magnetic fields are very small and we can ignore them and use electrostatic fields. We can easily see that the magnetic forces are small by writing down the electric and magnetic forces from a cylinder of charge moving down the $z$ axis. From Gauss' law, the radial electric field at a point inside the cylinder is

$$
E_{r}(r)=\frac{\rho r}{2 \epsilon_{0}}
$$

The force on a particle due to the electric field is then

$$
\mathbf{F}_{\text {electric }}=\frac{q}{m} E_{\tau} \hat{\mathbf{r}}
$$

where $\rho$ is the charge density and $\hat{r}$ is a unit normal in the radial direction. From Ampere's law, the magnetic field is

$$
B_{\theta}=\frac{\mu_{0} I_{\text {enclosed }}}{2 \pi r}
$$


The current enclosed in the loop can be written in terms of the charge density and the axial velocity as

$$
I_{\text {enclosed }}=\rho \pi r^{2} v_{z}
$$

So that the magnetic field can be written as

$$
B_{\theta}=\mu_{0} v_{z} \frac{\rho r}{2}=\mu_{0} \epsilon_{0} v_{z} E_{r}
$$

The force due to the magnetic field is then,

$$
\mathbf{F}_{\text {magnetic }}=\frac{q}{m} \frac{v_{z}^{2}}{c^{2}} E_{r} \hat{\mathbf{r}}=\frac{v_{z}^{2}}{c^{2}} \mathbf{F}_{\text {electric }}
$$

This tells us that the force on the particles due to the magnetic field is down by $(v / c)^{2}$ from the force due to the electric fields. In the beam frame $(v / c)$ is much, much less than $1 \%$ so the fields are electrostatic.

The scheme used to solve Poisson's equation in WARPrz is a slight variation on the method described in section 14-10 of Birdsall and Langdon[4]. In this method, we derive our difference scheme using the Gauss' law form of field equation because it avoids problems at the $r=0$ origin. Figure 2.2 shows the grid used in this method. Gauss' law is applied to the volume shown by the dashed line in the figure. The volume at the origin is done as a special case. The result is given in Birdsall and Langdon, section 14-10, equations (9) and (10)

$$
\begin{aligned}
\frac{Q_{j, k}}{\epsilon_{0}}= & 2 \pi \Delta z\left(r_{j+1 / 2} E_{r, j+1 / 2, k}-r_{j-1 / 2} E_{r, j-1 / 2, k}\right)+ \\
& \pi\left(r_{j+1 / 2}^{2}-r_{j-1 / 2}^{2}\right)\left(E_{z, j, k+1 / 2}-E_{z, j, k-1 / 2}\right)
\end{aligned}
$$

for $j \neq 0$ and

$$
\frac{Q_{0, k}}{\epsilon_{0}}=2 \pi r_{1 / 2} \Delta z E_{r, 1 / 2, k}+\pi r_{1 / 2}^{2}\left(E_{z, 0, k+1 / 2}-E_{z, 0, k-1 / 2}\right)
$$

where $r_{j+1 / 2}$ is the radius half way between the gridpoints, $r_{j+1 / 2}=1 / 2\left(r_{j+1}+r_{j}\right)$. Define the charge density as $\rho_{j, k}=Q_{j, k} / V_{j, k}$ where the volume is $V_{j, k}=\pi\left(\Delta r^{2}\right)_{j} \Delta z$ with $\left(\Delta r^{2}\right)_{j}=r_{j+1 / 2}^{2}-r_{j-1 / 2}^{2}$ for $j>0$ and $\left(\Delta r^{2}\right)_{0}=r_{1 / 2}^{2}$ for $j=0$. The grid spacing, 


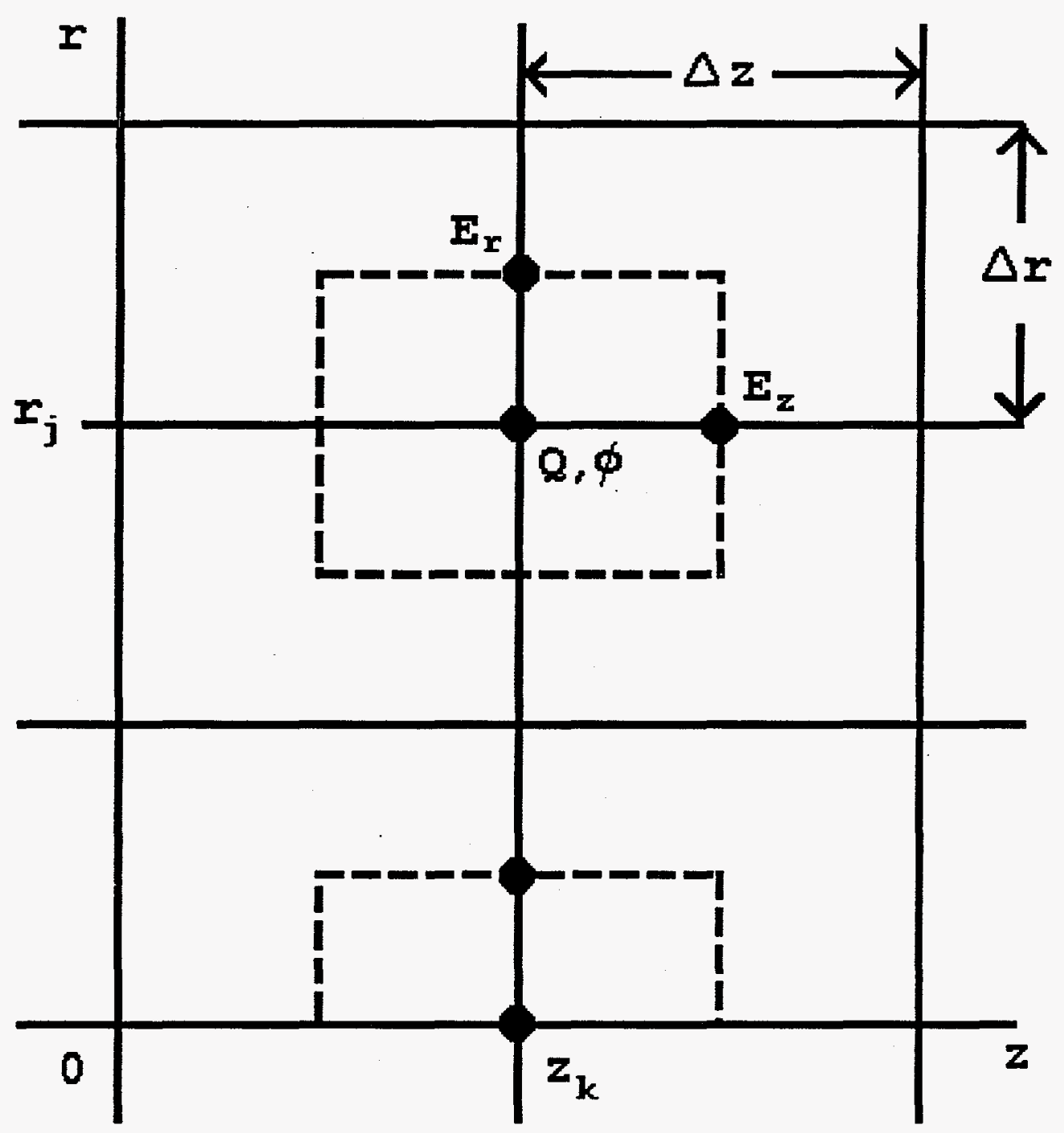

Figure 2.2: The grid used for the field solution. The dashed line indicates the volume used to calculate the density from the charge. 
$\Delta r$, is a constant. Use the finite difference approximation for the electric field from the electrostatic potential,

$$
\begin{aligned}
& E_{r, j+1 / 2, k}=-\frac{\phi_{j+1, k}-\phi_{j, k}}{\Delta r} \\
& E_{z, j, k+1 / 2}=-\frac{\phi_{j, k+1}-\phi_{j, k}}{\Delta z}
\end{aligned}
$$

We get a 5-point difference representation of Poisson's equation for $j>0$

$$
\begin{aligned}
-\frac{\rho_{j, k}}{\epsilon_{0}}= & \frac{2 r_{j+1 / 2}}{\left(\Delta r^{2}\right)_{j} \Delta r}\left(\phi_{j+1, k}-\phi_{j, k}\right)-\frac{2 r_{j-1 / 2}}{\left(\Delta r^{2}\right)_{j} \Delta r}\left(\phi_{j, k}-\phi_{j-1, k}\right)+ \\
& \frac{1}{\Delta z^{2}}\left(\phi_{j, k+1}-2 \phi_{j, k}+\phi_{j, k-1}\right)
\end{aligned}
$$

and for $j=0$

$$
-\frac{\rho_{0, k}}{\epsilon_{0}}=\frac{2 r_{1 / 2}}{\left(\Delta r^{2}\right)_{0} \Delta r}\left(\phi_{j+1, k}-\phi j, k\right)-\frac{1}{\Delta z^{2}}\left(\phi_{0, k+1}-2 \phi_{0, k}+\phi_{0, k-1}\right)
$$

where

$$
\left(\Delta r^{2}\right)_{j}=r_{j+1 / 2}^{2}-r_{j-1 / 2}^{2}
$$

and

$$
\left(\Delta r^{2}\right)_{0}=r_{1 / 2}^{2}
$$

For this field solution scheme it is only necessary to calculate the charge at each gridpoint-not the charge density. By introducing the charge density, we are dividing by the volume to get the density, then multiplying by the same volume in equation (2.23). We have chosen to calculate the charge density in WARPrz to keep the parallelism between the $r-z$ model and the $3 \mathrm{~d}$ model used in WARP3d which uses the charge density.

This is the scheme given in Birdsall and Langdon section 14-10. We slightly modify this scheme by using a fast Fourier transform in the $z$ direction. This is done by recognizing the last term in equation (2.23 as the finite difference approximation to the $\partial^{2} \phi / \partial z^{2}$ term in Poisson's equation. We can then replace this term by $-k^{2} \phi$ 
and replace $\phi$ and $\rho$ by their Fourier transforms. This gives as our final scheme: for $j>0$,

$$
-\frac{\tilde{\rho}_{j, k}}{\epsilon_{0}}=\frac{2 r_{j+1 / 2}}{\left(\Delta r^{2}\right)_{j} \Delta r}\left(\tilde{\phi}_{j+1, k}-\tilde{\phi}_{j, k}\right)-\frac{2 r_{j-1 / 2}}{\left(\Delta r^{2}\right)_{j} \Delta r}\left(\tilde{\phi}_{j, k}-\tilde{\phi}_{j-1, k}\right)-k_{z}^{2} \tilde{\phi}_{j, k}
$$

and for $j=0$

$$
-\frac{\tilde{\rho}_{0, k}}{\epsilon_{0}}=\frac{2 r_{1 / 2}}{\left(\Delta r^{2}\right)_{0} \Delta r}\left(\tilde{\phi}_{j+1, k}-\tilde{\phi}_{j, k}\right)-k_{z}^{2} \tilde{\phi}_{0, k}
$$

where $\tilde{\rho}$ and $\tilde{\phi}$ are the transformed quantities.

The general method used in the field solve routine is then:

1. Use an FFT routine to transform the charge density which was gotten from the accumulated particle data.

2. For each value of $k$, solve the tri-diagonal system given by equation (2.27) using the boundary conditions described below to get the electrostatic potential, $\tilde{\phi}$.

3. Use the inverse FFT routine to back transform this potential.

\section{Boundary Conditions for the Field Solve}

We have three boundary conditions available in WARPrz: a perfectly conducting wall, a wall with a resistance per unit length, and a wall with resistance and capacitance in parallel.

The perfectly conducting boundary is done by simply setting the electrostatic potential to zero along the wall $\left(r=r_{\text {wall }}\right)$

The wall with resistance or resistance and capacitance is used to study the longitudinal instability and will be discussed in chapter 3 .

\subsubsection{Interpolating the Field to the Particles}

Since WARPrz does not use excessive amounts of memory, we calculate the two components of the electric field from the potential and store them. The electric field 
components are

$$
\begin{aligned}
& E_{r, j, k}=-\frac{\phi_{j+1, k}-\phi_{j-1, k}}{2 \Delta r} \\
& E_{z, j, k}=-\frac{\phi_{j, k+1}-\phi_{j, k-1}}{2 \Delta z}
\end{aligned}
$$

The fields are then interpolated to the particle positions using the same area weighting used in the charge deposition and shown in figure (2.1). The fields at a particle $i p$ are then

$$
\begin{aligned}
& E_{r, i p}=\frac{A_{1}}{\Delta r \Delta z} E_{r, j, k}+\frac{A_{2}}{\Delta r \Delta z} E_{r, j+1, k+1}+\frac{A_{3}}{\Delta r \Delta z} E_{r, j+1, k}+\frac{A_{4}}{\Delta r \Delta z} E_{r, j, k+1} \\
& E_{z, i p}=\frac{A_{1}}{\Delta r \Delta z} E_{z, j, k}+\frac{A_{2}}{\Delta r \Delta z} E_{z, j+1, k+1}+\frac{A_{3}}{\Delta r \Delta z} E_{z, j+1, k}+\frac{A_{4}}{\Delta r \Delta z} E_{z, j, k+1}
\end{aligned}
$$

Unlike the charge deposition, the interpolation of the fields to the particles does vectorize. In WARPrz, we take the particles in groups of 256 and vectorize over that length.

\subsection{Diagnostics}

The major diagnostics used in WARPrz are described below. Many of the quantities computed directly during the PIC cycle are plotted at times specified by the user. Some of the most useful quantities include the electrostatic potential on the $r=0$ axis as a function of $z$, and the particle $\left(z, v_{z}\right)$ and $(r, z)$ phase spaces. Since WARPrz is built with BASIS, we can calculate any additional quantities and plot any quantity of interest through the interpreter.

\subsubsection{Line Charge Density}

The line charge density is

$$
\lambda(z)=\int_{0}^{2 \pi} \int_{0}^{a} \rho(r, z) r d r d \theta
$$

In the code, it is calculated by

$$
\lambda_{k}=2 \pi \sum_{j=0}^{n r} \rho_{j, k} r_{j} \Delta r
$$




\subsubsection{Beam Current}

The $z$ component of the current is calculated using a one dimensional weighting scheme so that the contribution from the $i p$ th particle to the current is

$$
\begin{gathered}
I_{k}=\frac{L_{1}}{\Delta z} w q u_{z, i p} \\
I_{k+1}=\frac{L_{2}}{\Delta z} w q u_{z, i p}
\end{gathered}
$$

where $L_{1}$ is the distance between the particle and the axial grid point $z_{k+1}$ and $L_{2}$ is the distance between the particle and the axial gridpoint $z_{k}$.

\subsubsection{Particle Moments}

Many moments of the particle distribution are taken. Among the most useful for the longitudinal work in WARPrz are the average $v_{z}$

$$
\bar{v}_{z}=\frac{1}{N} \sum_{i p=0}^{N} v_{z, i p}
$$

and the RMS $v_{z}$ and $v_{r}$

$$
v_{z, \mathrm{RMS}}=\sqrt{\overline{v_{z}^{2}-\bar{v}_{z}^{2}}}
$$

with $\overline{v_{z}^{2}}$

$$
\overline{v_{z}^{2}}=\frac{1}{N} \sum_{i p=0}^{N} v_{z, i p}^{2}
$$

and $v_{r, \text { RMS }}$ calculated in the same way using $v_{r}$ instead of $v_{z}$.

These moments can be calculated over various subsets of particles. They are calculated as a function of $z$, with particles contributing to the moments at nearest gridpoints. The user can also specify slices or windows in $z$ and moments are calculated based on the particles inside these windows. 


\section{Chapter 3}

\section{Model for the Module Impedance}

In order to study the longitudinal instability with WARPrz, we need a model for the module impedance in the code. We have chosen to model the modules with a continuous gap approximation, as a resistive wall. In this approximation, the impedance of the gap is applied as an impedance per unit length. Thus a module which has gap impedance of $100 \mathrm{Ohms}$ and total length (gap length plus nongap length) of 1 meter would be represented as a wall with a continuous $100 \mathrm{Ohm} / \mathrm{meter}$ resistance. This model contains all the relevant physics for the low frequency longitudinal instability and compares nicely with analytic work. It does not include gap transit time effects or module resonances.

\subsection{Model for the Resistive Wall in WARPrz}

We formulate our model first for a purely resistive wall since this is the simpler case. We generalize this model to include capacitance in section 3.3.

We could include the resistive wall component of the electric field using the beam current as we did in the fluid model $(E=-\eta I)$. In a particle-in-cell code, however, the current is calculated by summing over the particles. Unless there are a very large number of particles in each cell, the current tends to be a noisy quantity. Instead, we want to calculate the resistive wall contribution to the electric field using the Poisson 
solve at the boundary. There is some smoothing of high frequencies which takes place because a finite number of Fourier modes. By using the electrostatic potential at the boundary to calculate the resistive wall part of the electric field we will get a smoother, less noisy, more physical result.

The model is derived by assuming a continuity equation for the wall surface charge, $\sigma$, which has units of charge/area.

$$
\frac{\partial \sigma}{\partial t}+\frac{\partial K_{z}}{\partial z}=0
$$

where $K_{z}$ is the surface current. We also have Ohm's law, $2 \pi b \eta K_{z}=E_{z}$, where $\eta$ is the resistance per unit length. Substituting this into the continuity equation yields

$$
\frac{\partial \sigma}{\partial t}=-\frac{\partial}{\partial z}\left(\frac{E_{z}}{2 \pi b \eta}\right)
$$

Integrating this equation over the surface and assuming cylindrical symmetry gives

$$
\frac{\partial}{\partial t} \int_{z_{1}}^{z_{2}} \sigma d z=-\frac{1}{2 \pi b \eta}\left[E_{z}\left(z_{2}\right)-E_{z}\left(z_{1}\right)\right]
$$

where we have taken the limits on $z$ to be $z_{1}$ to $z_{2}$. Define the electrostatic potential, $\phi$, by

$$
E_{z}\left(z^{\prime}\right)=-\left.\frac{\partial \phi}{\partial z}\right|_{z^{\prime}}
$$

Consider the case of $z_{2}=z_{1}+\Delta z$ where $\Delta z$ is small. Over this range, we can assume that $\sigma$ doesn't change much and we can evaluate the integral over $\sigma$ giving us

$$
\frac{\partial}{\partial t} \int_{z_{1}}^{z_{1}+\Delta z} \sigma d z=\Delta z \frac{\partial \sigma}{\partial t}
$$

Defining the surface charge, ${ }^{s} Q$, as ${ }^{s} Q=2 \pi b \Delta z \sigma$, and using the results of equations (3.4) and (3.5) in equation (3.3) gives

$$
\frac{\partial\left({ }^{s} Q\right)}{\partial t}=\frac{1}{\eta}\left[\left.\frac{\partial \phi}{\partial z}\right|_{z_{1}+\Delta z}-\left.\frac{\partial \phi}{\partial z}\right|_{z_{1}}\right]
$$

Since we have assumed that $\Delta z$ is small, we can expand $\partial \phi / \partial z$ in a Taylor series. Taking the first two terms of this expansion and substituting into equation (3.6) gives the result

$$
\frac{\partial\left({ }^{s} Q\right)}{\partial t}=\frac{\Delta z}{\eta} \frac{\partial^{2} \phi}{\partial z^{2}}
$$


This equation is then solved simultaneously with Poisson's equation in the code.

The solve is done by . Fourier transforming equation (3.7) with respect to $z$ and then using a finite difference approximation to the time derivative. Doing this gives,

$$
{ }^{s} \tilde{Q}^{n+1}={ }^{s} \tilde{Q}^{n}-\frac{\Delta t \Delta z}{\eta} k^{2} \tilde{\phi}^{n+1}
$$

where the superscript denotes the time level and the tilde denotes the Fourier transform. We can write Poisson's equation as

$$
\left(\nabla_{r}^{2}-k^{2}\right) \tilde{\phi}^{n+1}=-\frac{\tilde{\rho}^{n+1}}{\epsilon_{0}}
$$

where $\nabla_{\tau}^{2}$ is the radial part of $\nabla^{2}$. At the wall, $\tilde{\rho}^{n+1}$ is composed of the charge density due to the beam plus the charge density due to the surface charge, ${ }^{s} \tilde{Q}^{n+1}$. We substitute for ${ }^{s} \tilde{Q}^{n+1}$ in Poisson's equation at the wall from equation (3.8) and are left with an equation for $\tilde{\phi}^{n+1}$ in terms of the surface charge at the last time step, ${ }^{s} \tilde{Q}^{n}$, and the plasma density. The radial part of the Laplacian is differenced to give a tridiagonal system of equations for each value of $k$. These systems are solved via Gaussian elimination using the boundary condition that the radial electric field is zero for $r>r_{\text {wall }}$ to give $\tilde{\phi}^{n+1}$. We then update the surface charge using equation (3.8).

The above derivation does not take into account the fact that we are doing the calculation in a moving window. To take this into account, we advect the surface charge backward in the window. Currently we use a simple advection scheme. We require that the time step be such that we move an integer number of grid cells per step; that is, $\Delta t=N \Delta z / v_{\text {beam }}$ where $\mathrm{N}$ is an integer. If this is time step criterion is met, then the advection of the surface charge becomes a simple remapping since our system is assumed to be periodic. Figure (3.1) shows this remapping.

In this derivation, we have assumed that the radial electric field is zero outside the wall. In section 3.4, we discuss how one could build such a wall. 


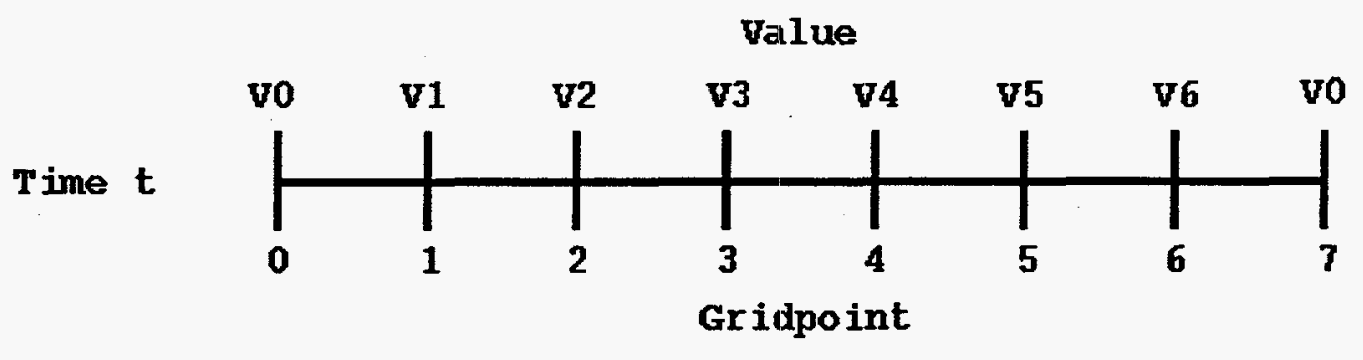

Time $t+d t$

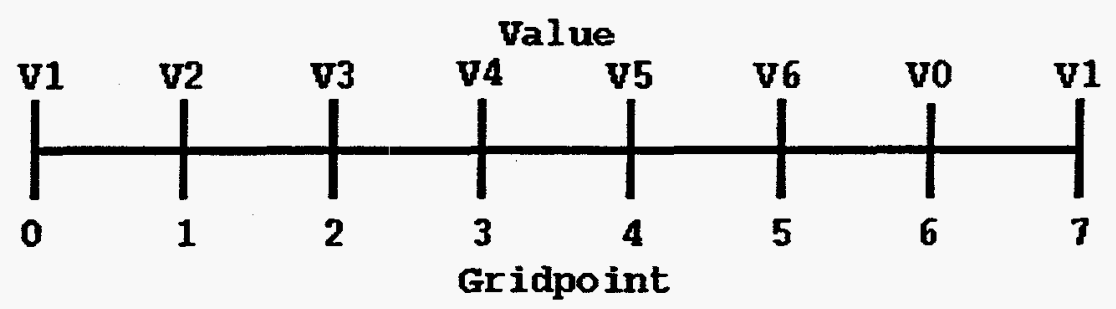

Figure 3.1: The advection is just a remapping of the values to the correct array index if the mesh moves by an integer number of grid cells per timestep

\subsection{Comparing the Model to 1-d Theory}

We require our model reduce to the simpler model used in the 1-d fluid model in the long wavelength limit. Recall equation (3.2) which was used to derive the wall model for WARPrz:

$$
\frac{\partial \sigma}{\partial t}=-\frac{\partial}{\partial z}\left(\frac{E_{z}}{2 \pi b \eta}\right)
$$

Fourier transform this equation in space and time and note that the lab frame frequency must be used

$$
2 \pi b \sigma \eta\left(\omega^{\prime}+k v_{b}\right)=k E_{z}
$$

where the prime on the $\omega$ indicates that it is the beam frame frequency.

In the long wavelength limit the line charge density can be written as $\lambda \approx-2 \pi b \sigma$. We also note that the phase velocity in the beam frame $\left(\omega^{\prime} / k \approx v_{p}\right)$ is small compared 
with the beam velocity $\left(v_{\text {beam }}\right)$. This leaves

$$
E_{z}=-\eta v_{b} \lambda
$$

In terms of the beam current, $I=v_{b} \lambda$,

$$
E_{z}=-\eta I
$$

which is the contribution to the electric field that we used in the linear analysis in equation (1.12). We conclude that our model reduces to the simple model used in the linear theory in the long wavelength limit.

\subsection{Adding the Capacitive Component of the Mod- ule Impedance}

The capacitive part of the accelerating module impedance can be added to the derivation of section 3.1. From elementary circuit theory,

$$
I=\mathcal{C} \frac{d V}{d t}
$$

If we use this and add a capacitor and a resistor in parallel we get an Ohm's law:

$$
2 \pi b \eta K_{z}=E_{z}+\eta \mathcal{C} \frac{d E_{z}}{d t}
$$

where $\mathrm{b}$ is the pipe radius, $\eta$ is the resistance per unit length, and $\eta \mathcal{C}$ is the " $\mathrm{RC}$ " time. If we let $K_{z}, E_{z} \approx \exp (-i \omega t)$ then we get an impedance,

$$
Z(\omega)=\frac{\eta}{1-i \omega \eta \mathcal{C}}
$$

which is the impedance of a resistor and capacitor in parallel given that we have chosen a minus sign in the exponential. We can then go through the same derivation with this Ohm's law that we did when we had resistance alone. This gives the result:

$$
\frac{\partial \sigma}{\partial t}=\frac{1}{2 \pi b} \frac{\partial}{\partial z}\left[\frac{1}{\eta}\left(\frac{\partial \phi}{\partial z}+\eta \mathcal{C} \frac{d}{d t} \frac{\partial \phi}{\partial z}\right)\right]
$$


where $\sigma$ is the surface charge density and has units of charge/area. By saving $\phi$ at the previous time step, we can use a finite difference approximation for the time derivatives and then solve simultaneously with the Poisson equation. We also define the surface charge, ${ }^{s} Q=2 \pi b \Delta z \sigma$.

In the case of $\eta$ and $\eta \mathcal{C}$ constant, we can Fourier Transform this equation in $z$ and we get

$$
\frac{s \tilde{Q}_{k}^{n+1}-s \tilde{Q}_{k}^{n}}{\Delta t}=-\frac{\Delta z}{\eta} k_{z}^{2}\left(\tilde{\phi}_{r_{w}, k}^{n+1}+\eta \mathcal{C} \frac{\tilde{\phi}_{\tau_{w}, k}^{n+1}-\tilde{\phi}_{r_{w}, k}^{n}}{\Delta t}\right)
$$

where $\tilde{Q}$ and $\tilde{\phi}$ are the Fourier transforms of $Q$ and $\phi$.

\subsection{A Physical Picture for This Model}

The model that we have used to approximate the impedance of the accelerating modules is that of a wall with a continuum of resistors and capacitors in parallel. When we solve Poisson's equation with the resistive wall, we've also assumed that the radial electric goes to zero for radii larger than the wall radius. Although this system is an approximation to the impedance of the accelerating gaps, we wonder if it is possible to build such a device. In this section, we will discuss a physical model for our continuum approximation proposed by $\mathrm{E}$. P. Lee.

The device under consideration is a cylindrical tube which is filled with metal irises. The tube wall has a resistance per unit length, $\eta$. The irises have an inner radius of $b$ and an outer radius of $r_{0}$ (see figures (3.2) and (3.3)). This inner radius (b) corresponds to the wall in the WARPrz code. The space between the irises can be filled with a dielectric material. We want to show that this configuration has an impedance of the form,

$$
Z(\omega)=\frac{\eta}{1-i \omega \eta \mathcal{C}}
$$

where $\eta$ is the resistance and $\eta \mathcal{C}$ is the "RC" decay time in the low frequency limit ( $\omega<c_{m} / r_{0}$ where $c_{m}$ is the speed of light in the dielectric material). 


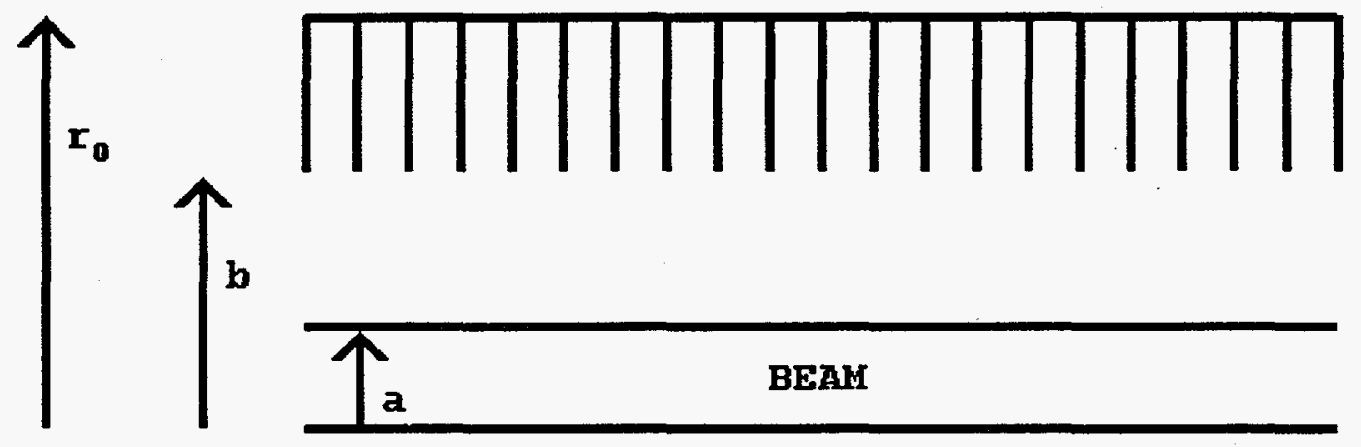

Figure 3.2: Side view of iris structure. Structure is cylindrically symmetric about center axis

\subsubsection{Calculating the Radial Electric Field Between Irises}

We want to show that if the irises are close together, the radial electric field is shorted out in the space between the irises. Consider the case of a conducting tube with two metal irises as is shown in figure (3.4). We want to show that between that if the irises are close together, the radial electric field between the irises is close to zero.

From physical intuition we guess that the radial field is zero between the irises if the distance between irises (b) is small compared with the radial depth of the iris $\left(r_{0}-b\right)$. When this is the case, physical intuition leads us to suspect that the radial field will be shorted out.

To verify this mathematically, consider Poisson's equation in the region between irises.

$$
\frac{1}{r} \frac{\partial}{\partial r} r \frac{\partial \phi}{\partial r}+\frac{\partial^{2} \phi}{\partial z^{2}}=0
$$




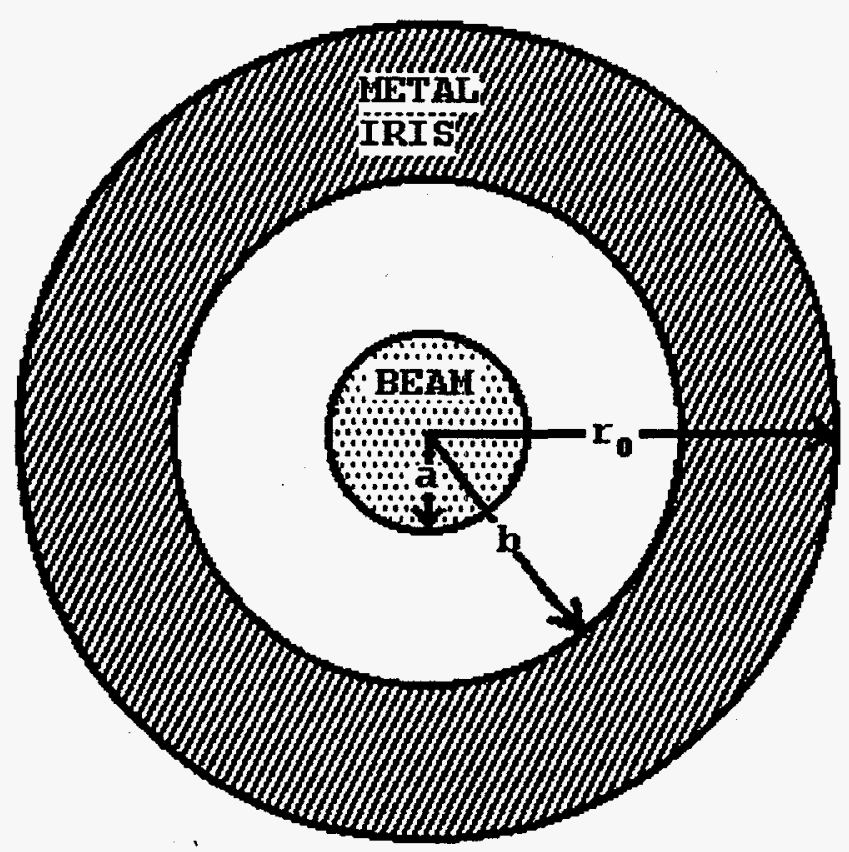

Figure 3.3: End view of iris structure

We separate variables by assuming $\phi$ has the form

$$
\phi(r, z)=R(r) Z(z)
$$

Substituting this in and using the usual tricks gives us equations for $Z(z)$ and $R(r)$

$$
\begin{gathered}
\frac{\partial^{2} Z(z)}{\partial z^{2}}+k^{2} Z(z)=0 \\
\frac{1}{r} \frac{\partial}{\partial r} r \frac{\partial R(r)}{\partial r}-k^{2} R(r)=0
\end{gathered}
$$

where $k^{2}$ is the separation constant.

Equation (3.21) has a solution of sines and cosines. The boundary conditions in $z$ are that $\phi=0$ at $z=0$ and $z=h$. This leads us to

$$
Z(z)=\sin (k z)
$$

and

$$
k=\frac{n \pi}{h}
$$




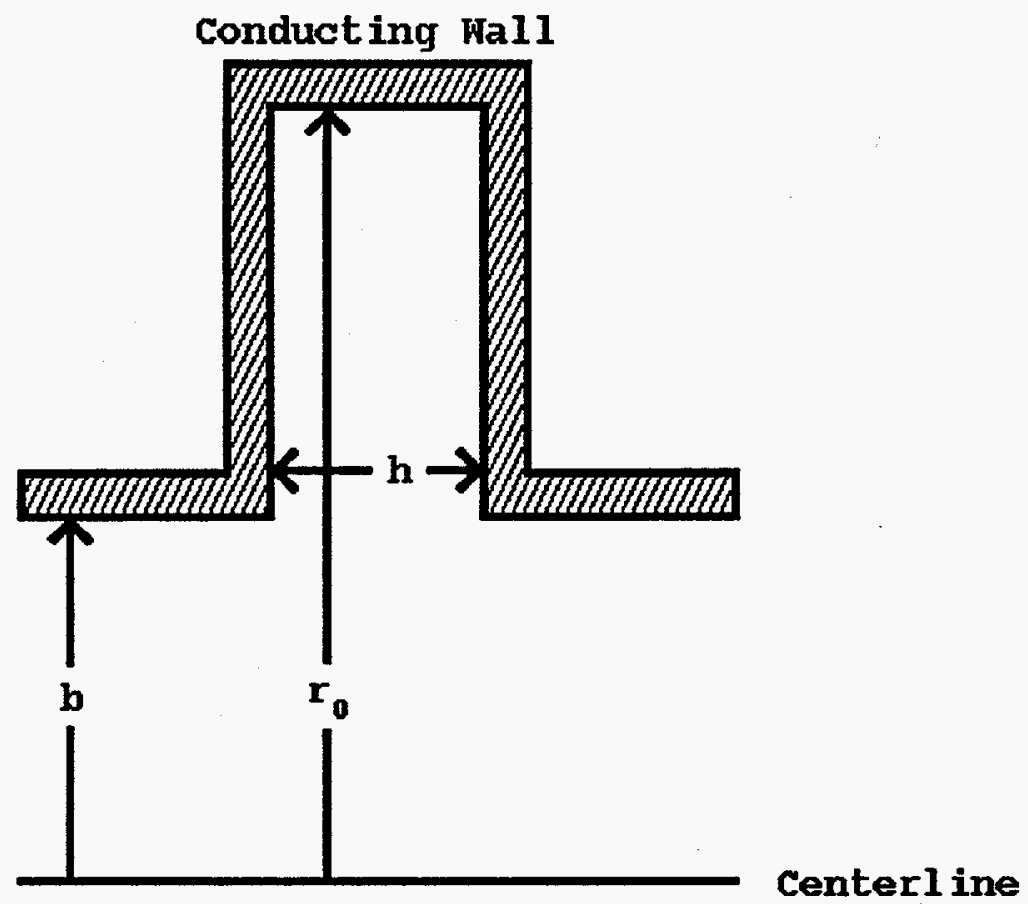

Figure 3.4: Side view close up of a single pair of irises and their dimensions where $n$ is an integer.

The radial equation (equation (3.22)) is a modified Bessel equation. This leads to the solution

$$
\phi(r, z)=A \sin (k z)\left[B I_{0}(k r)+K_{0}(k r)\right]
$$

where $A$ and $B$ are constants. At the boundary $r=r_{0}, \phi=0$ since we've assumed a conducting wall there. This allows us to solve for the constant $B$,

$$
B=-\frac{K_{0}\left(k r_{0}\right)}{I_{0}\left(k r_{0}\right)}
$$

Using this, we can write the electrostatic potential as

$$
\phi(r, z)=\frac{A \sin (k z)}{I_{0}\left(k r_{0}\right)}\left[K_{0}(k r) I_{0}\left(k r_{0}\right)-K_{0}\left(k r_{0}\right) I_{0}(k r)\right]
$$

and the radial electric field as

$$
E_{\tau}=k A \sin (k z) \frac{\left[K_{1}(k r) I_{0}\left(k r_{0}\right)+K_{0}\left(k r_{0}\right) I_{1}(k r)\right]}{I_{0}\left(k r_{0}\right)}
$$




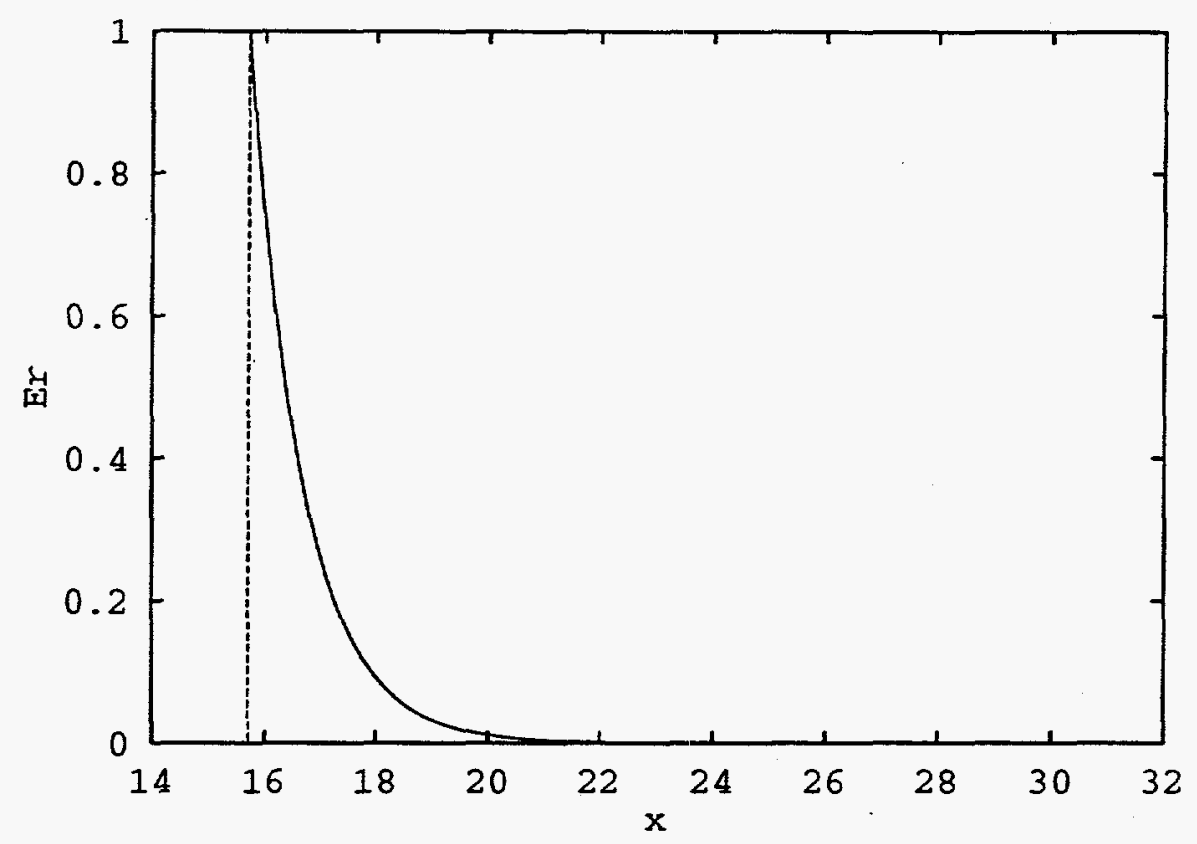

Figure 3.5: The radial electric field (as a fraction of its value at the inner radius) as a function of $x=n \pi r / h$ for irises 5 times deeper than long. The dashed, vertical line shows the position of the inner radius at $x_{0}=5 \pi$.

Figures (3.5), (3.6), and (3.7) show plots of $\left[K_{1}(x) I_{0}\left(x_{0}\right)+K_{0}\left(x_{0}\right) I_{1}(x)\right] / I_{0}\left(x_{0}\right)$ which is proportional to the radial electric field as a function of $x=n \pi r / h$ for various values of $x_{0}=n \pi r_{0} / h$. In each case, we have kept the inner radius of the irises (b) fixed such that $x_{\min }=n \pi b / h=5 \pi$.

Figure (3.5) shows the relative radial electric field when $x_{0}=10 \pi$. This corresponds to an iris which has a radial depth $\left(r_{0}-b\right)$ of five times its length $(h)$. We see that the radial electric field falls off to $10 \%$ of the value at $r=b$ about $15 \%$ of the distance into the irises.

Figure (3.6) shows the relative radial electric field when $x_{0}=5.5 \pi$. This corresponds to an iris which has a radial depth $\left(r_{0}-b\right)$ of $1 / 2$ its length $(h)$. Our physical intuition was that the radial field would never be very small in this case and the figure bears this out. The radial field never falls below $30 \%$ of its value at $r=b$.

Figure (3.7) shows the field for $x_{0}=50 \pi$, which is an iris with depth 45 times its 


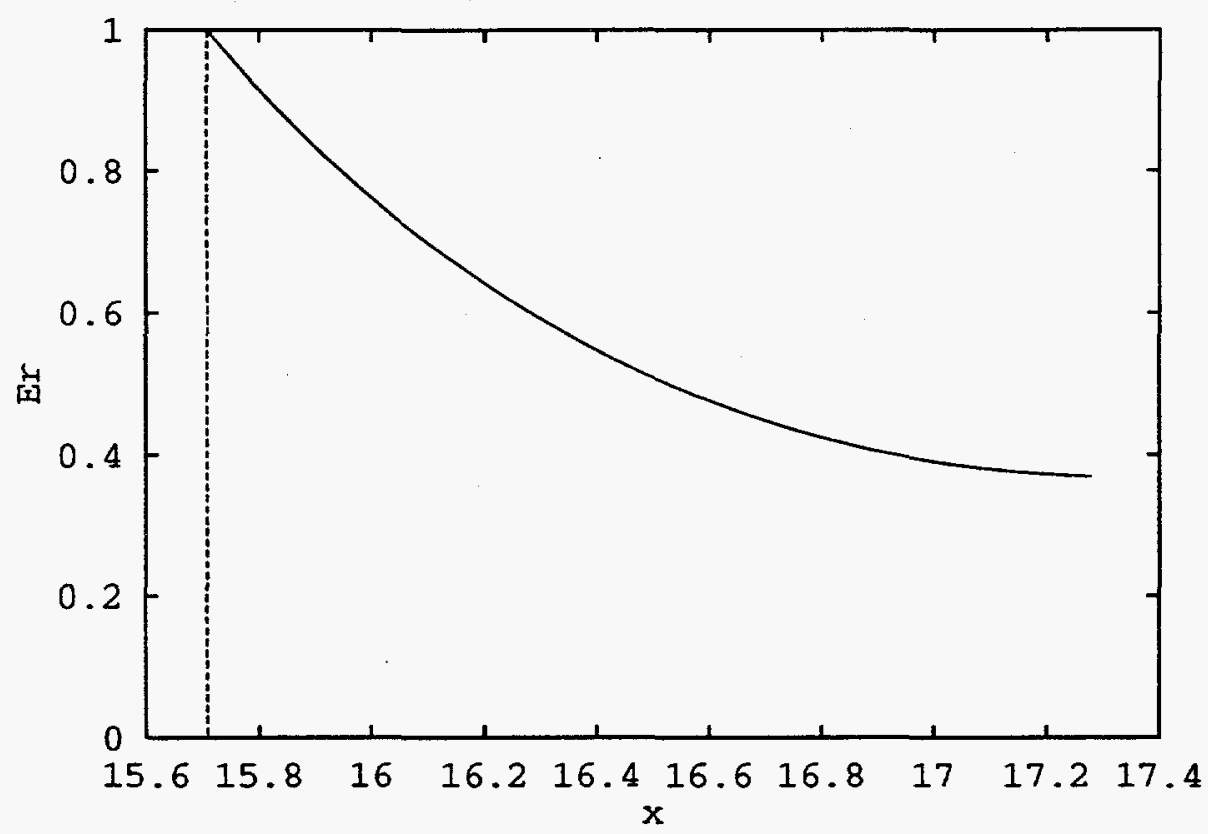

Figure 3.6: The radial electric field (as a fraction of its value at the inner radius) as a function of $x=n \pi r / h$ for irises half as deep as long. The dashed, vertical line shows the position of the inner radius at $x_{0}=5 \pi$.

length. In this extreme case, we see that the radial electric field falls off very quickly compared with the length.

\subsubsection{Calculating the Impedance of the Iris Structure}

We now know that if the irises are close together the radial electric field in the region $b<r<r_{0}$ is shorted out and we can ignore it when we calculate the impedance of the iris structure. If we take advantage of the cylindrical symmetry of the system, there are only only two components of the electric and magnetic fields and two Maxwell's equations to solve between the irises.

$$
\begin{gathered}
-\frac{\partial E_{z}}{\partial r}=-\frac{\partial B_{\theta}}{\partial t} \\
\frac{1}{r} \frac{\partial\left(r B_{\theta}\right)}{\partial r}=\epsilon \mu_{0} \frac{\partial E_{z}}{\partial t}
\end{gathered}
$$




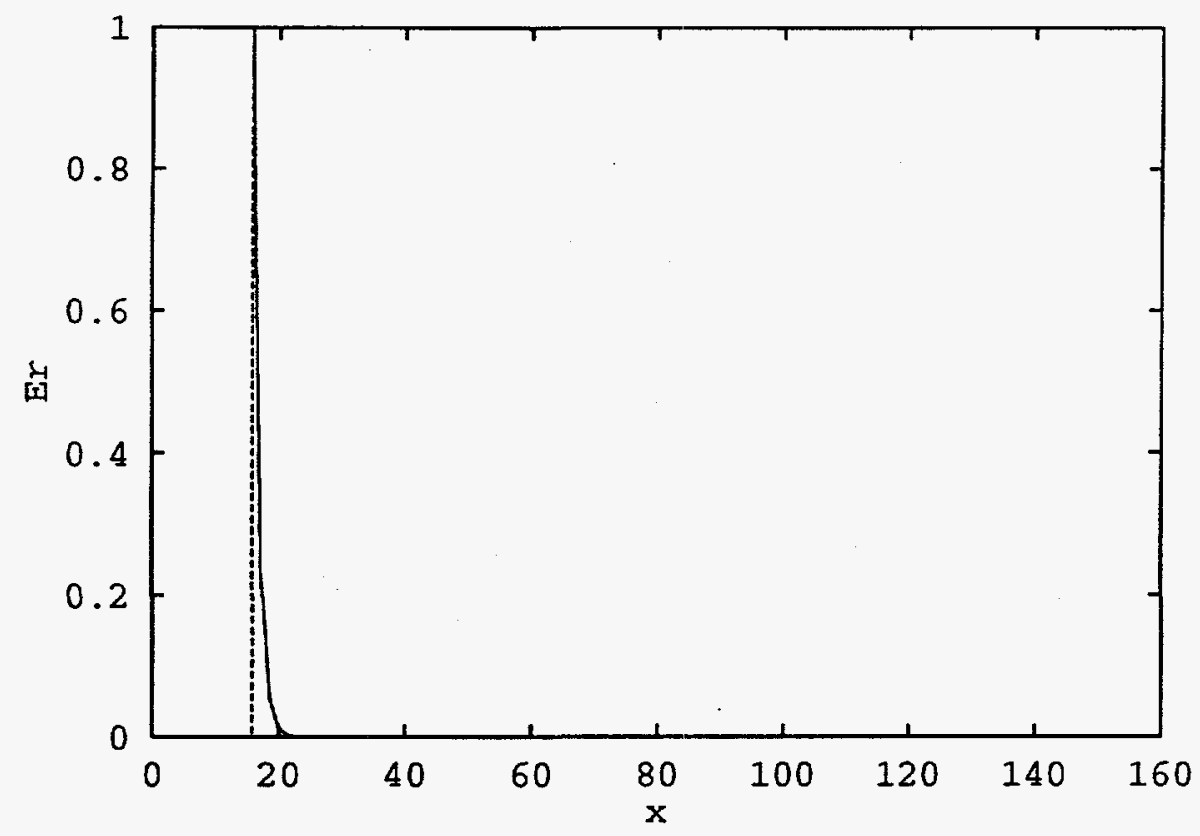

Figure 3.7: The radial electric field (as a fraction of its value at the inner radius) as a function of $x=n \pi r / h$ for irises 45 times deeper than long. The dashed, vertical line shows the position of the inner radius at $x_{0}=5 \pi$

We assume that $E_{z}$ and $B_{\theta}$ vary as $\exp (-i \omega t)$ and combine the equations.

$$
\frac{1}{r} \frac{\partial}{\partial r} r \frac{\partial E_{z}}{\partial r}+\omega^{2} \epsilon \mu_{0} E_{z}=0
$$

This gives us Bessel functions as the solution,

$$
E_{z}=A J_{0}(x)+B N_{0}(x)
$$

where

$$
x=\omega r / c_{m}
$$

and $c_{m}$ is the speed of light in the dielectric material, $c_{m}=1 / \sqrt{\epsilon \mu_{0}}$. The magnetic field is given by

$$
B_{\theta}=\frac{i}{\omega} \frac{\partial E_{z}}{\partial r}=\frac{i}{c_{m}} \frac{\partial E_{z}}{\partial x} .
$$

Which becomes

$$
B_{\theta}=\frac{-i}{c_{m}}\left(A J_{1}(x)+B N_{1}(x)\right),
$$


where we use $J_{0}^{\prime}=-J_{1}$ and $N_{0}^{\prime}=-N_{1}$.

At $r=r_{0}$, there is a resistive tube (neglected in section 3.4.1) which leads to the boundary condition, $E_{z}=2 \pi r_{0} \eta K$ where $K$ is the surface current. The other boundary condition comes from the discontinuity in the magnetic field where the surface current is located and is given by $-B_{\theta}=\mu_{0} K$ where we assume the magnetic field outside is zero. These boundary conditions lead to conditions on the constants $A$ and $B$,

$$
\begin{gathered}
2 \pi r_{0} \eta K=A J_{0}\left(x_{0}\right)+B N_{0}\left(x_{0}\right) \\
\mu_{0} c_{m} K=i\left(A J_{1}\left(x_{0}\right)+B N_{1}\left(x_{0}\right)\right)
\end{gathered}
$$

where $x_{0}$ is $x$ evaluated at $r=r_{0}$.

Solving these two equations for $A$ and $B$ gives,

$$
A / K=\frac{2 \pi r_{0} \eta}{J_{0}\left(x_{0}\right)}-\frac{N_{0}\left(x_{0}\right)}{J_{0}\left(x_{0}\right)} \frac{\pi x_{0}}{2}\left(2 \pi r_{0} \eta J_{1}\left(x_{0}\right)+i \mu_{0} c_{m} J_{0}\left(x_{0}\right)\right)
$$

and

$$
B / K=\frac{\pi x_{0}}{2}\left(2 \pi r_{0} \eta J_{1}\left(x_{0}\right)+i \mu_{0} c_{m} J_{0}\left(x_{0}\right)\right)
$$

where we use

$$
J_{1}(z) N_{0}(z)-J_{0}(z) N_{1}(z)=2 / \pi z
$$

Using these expressions, we get the electric and magnetic fields.

$$
\begin{aligned}
E_{z} / K= & \frac{2 \pi r_{0} \eta}{J_{0}\left(x_{0}\right)}\left[1-\frac{\pi x_{0}}{2} N_{0}\left(x_{0}\right) J_{1}\left(x_{0}\right)\right] J_{0}(x)+\pi^{2} x_{0} r_{0} \eta J_{1}\left(x_{0}\right) N_{0}(x) \\
& +i \frac{\pi x_{0}}{2} \mu_{0} c_{m}\left(J_{0}\left(x_{0}\right) N_{0}(x)-N_{0}\left(x_{0}\right) J_{0}(x)\right) \\
c_{m} B_{\theta} / K= & -i \frac{2 \pi r_{0} \eta}{J_{0}\left(x_{0}\right)}\left[1-\frac{\pi x_{0}}{2} N_{0}\left(x_{0}\right) J_{1}\left(x_{0}\right)\right] J_{1}(x)-i \pi^{2} x_{0} r_{0} \eta J_{1}\left(x_{0}\right) N_{1}(x) \\
& +\frac{\pi x_{0}}{2} \mu_{0} c_{m}\left(J_{0}\left(x_{0}\right) N_{1}(x)-N_{0}\left(x_{0}\right) J_{1}(x)\right)
\end{aligned}
$$

The impedance can be calculated from the magnetic and electric fields at $r=b$ by noting that the impedance is

$$
Z=-E_{z}(r=b) / I
$$




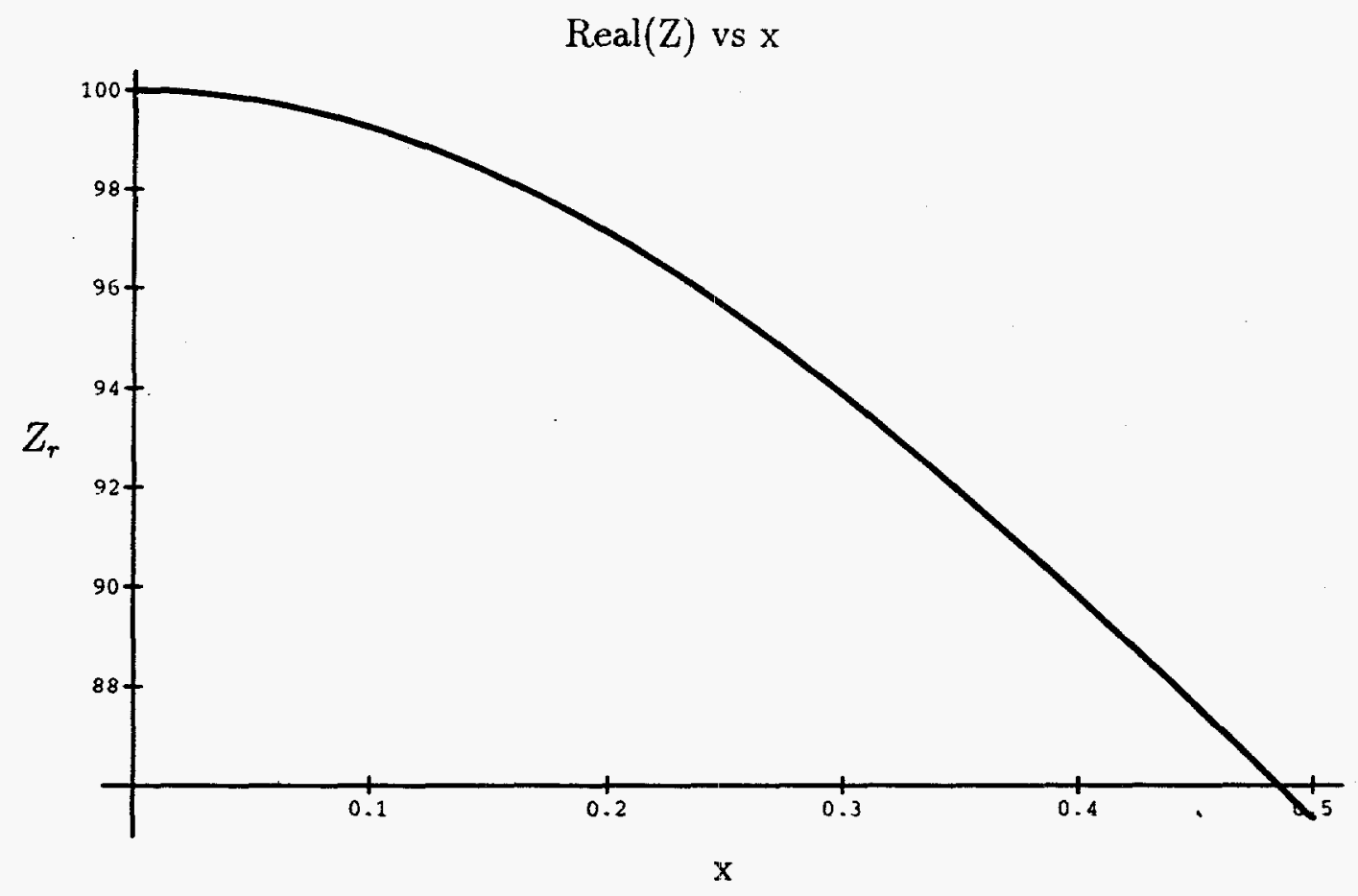

Figure 3.8: The real part of the impedance approaches $100 \mathrm{Ohms} / \mathrm{m}$ as $\mathrm{x}$ (proportional to frequency) gets small

while the beam current $(I)$ is related to the magnetic field,

$$
B_{\theta}(r=b)=\frac{\mu_{0}}{2 \pi b} I
$$

Combining these equations gives an expression for the impedance

$$
Z=-\frac{\mu_{0}}{2 \pi b} \frac{E_{z}(r=b)}{B_{\theta}(r=b)}
$$

Figures (3.8 - 3.9) show the real and imaginary parts of the impedance as a function of $x$ (which is proportional to the frequency) for $x_{0}=2 x$, vacuum in the gaps between the irises, a wall resistance of $100 \mathrm{Ohms} / \mathrm{m}$ and a wall radius $\left(r_{0}\right)$ of 1 meter.

\subsubsection{Low Frequency Limit}

In the low frequency limit, we show that the irises have an impedance made up of a resistor and capacitor in parallel like we use in WARPrz. Recall that a resistor and 


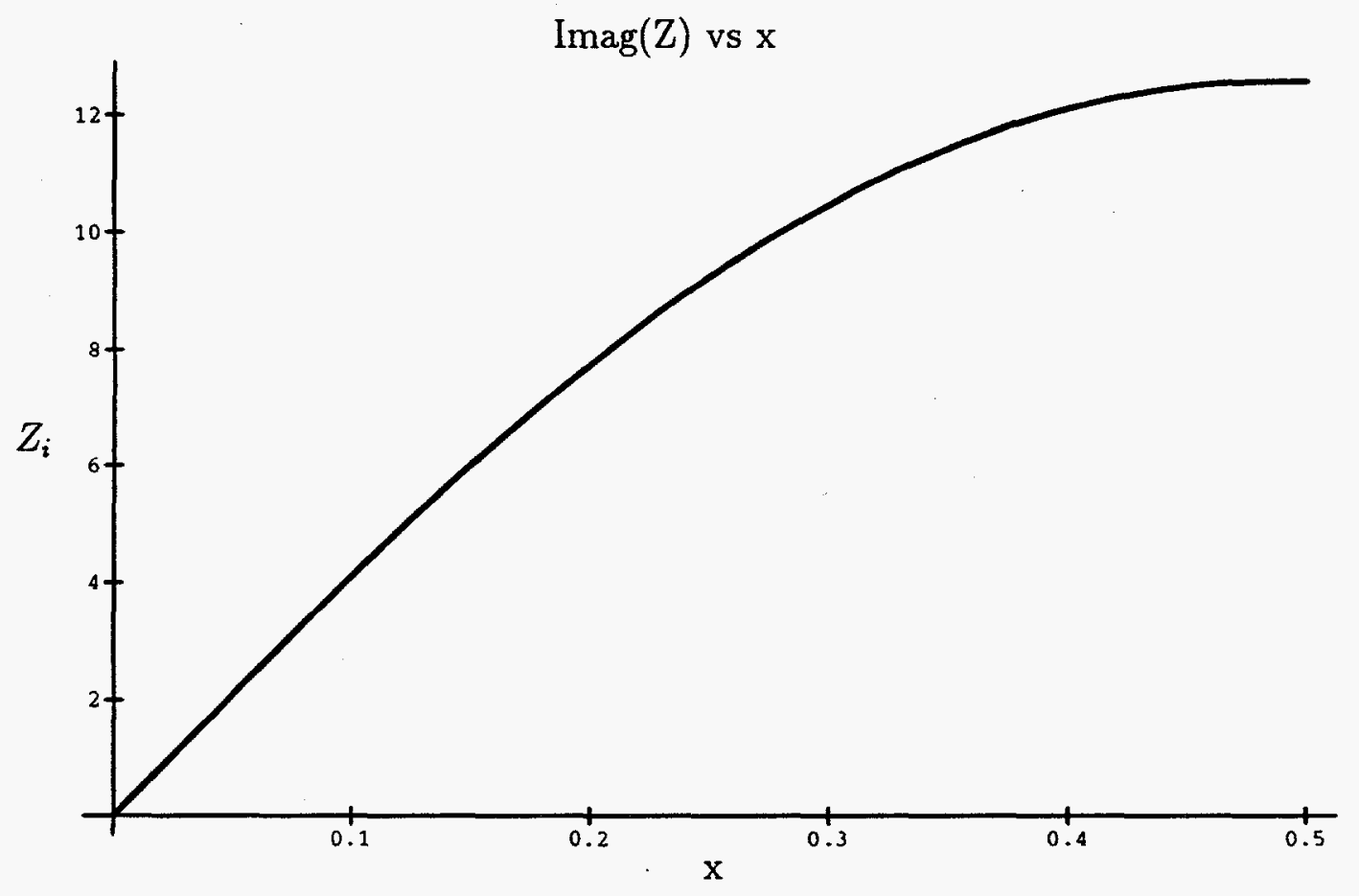

Figure 3.9: The imaginary part of the impedance becomes linear as $\mathrm{x}$ (proportional to frequency) gets small

capacitor in parallel have the impedance

$$
Z(\omega)=\frac{\eta}{1-i \omega \eta \mathcal{C}}
$$

Rationalizing the impedance gives

$$
Z(\omega)=\frac{\eta+i \omega \eta^{2} \mathcal{C}}{1+\omega^{2} \eta^{2} \mathcal{C}^{2}}
$$

We expect, then, that for low frequencies that the real part of the impedance. will be a constant equal to $\eta$ and the imaginary part will increase linearly with frequency. Figures $(3.8-3.9)$ indeed show this since the real part approaches 100 and the imaginary part is linear with $x$ which is proportional to the frequency. For a more detailed look at the low frequency regime, we expand the Bessel functions to get an analytic expression for the low frequency limit.

The leading terms of the Bessel functions we need are:

$$
J_{0}(x) \simeq 1
$$




$$
\begin{gathered}
J_{1}(x) \simeq x / 2 \\
N_{0}(x) \simeq 2 / \pi[\gamma+\ln (x / 2)] \\
N_{1}(x) \simeq-2 /(\pi x)
\end{gathered}
$$

Keeping terms to first order in $x$ and/or $x_{0}$ (i.e. to first order in frequency) in equations (3.41) and (3.42), gives the electric and magnetic fields are $r=b$,

$$
\begin{gathered}
\frac{c_{m} B_{\theta}(r=b)}{K}=-\mu_{0} c_{m} \frac{r_{0}}{b}+i \frac{\pi r_{0} \eta \omega}{b c_{m}}\left(r_{0}^{2}-b^{2}\right) \\
\frac{E_{z}(r=b)}{K}=2 \pi r_{0} \eta-i \mu_{0} r_{0} \omega \ln \left(\frac{r_{0}}{b}\right)
\end{gathered}
$$

We can use these expressions for the electric and magnetic fields to calculate the impedance in equation (3.45)

$$
Z=-\frac{\mu_{0}}{2 \pi b} \frac{E_{z}(r=b)}{B_{\theta}(r=b)}=-\frac{\mu_{0} c_{m}}{2 \pi b} \frac{\left[2 \pi r_{0} \eta-i \mu_{0} r_{0} \omega \ln \left(r_{0} / b\right)\right]}{\left[-\mu_{0} c_{m}\left(r_{0} / b\right)+i \pi r_{0} \eta \omega\left(r_{0}^{2}-b^{2}\right) /\left(b c_{m}\right)\right]}
$$

Simplifying

$$
Z=\frac{\eta-i \omega \mu_{0} \ln \left(r_{0} / b\right) /(2 \pi)}{1-i \omega \eta \epsilon \pi\left(r_{0}^{2}-b^{2}\right)}
$$

At this point, we need to stop and consider our choice of units for the circuit elements in this problem. In both WARPrz and the iris calculation, we have expressed the resistance as a resistance per length (Ohms/meter) so that

$$
\eta=R / h
$$

where $R$ is the resistance (units of Ohms), $\eta$ is the resistance per length (units of Ohms per meter) and $h$ is the distance between irises. As a consequence of this choice, the capacitance used in WARPrz and the iris model is a capacitance times the distance between irises,

$$
\mathcal{C}=C h
$$

where $\mathrm{C}$ has units of Farad-meters and $C$ has units of Farads. This choice keeps $\eta \mathcal{C}$ as the "RC" time. Although we don't include inductance in the WARPrz code, the expressing resistance per length leaves the inductance as

$$
\mathcal{L}=L / h
$$


where $\mathcal{L}$ has units of henries per meter while $L$ has units of henries.

We now want to identify the terms in equation (3.55) in terms of the resistance, capacitance and inductance of the iris structure. The iris structure is a series of parallel plate capacitors. The capacitance of a set of parallel plates is

$$
C=\epsilon(\text { Area }) / h
$$

where the area is just the area of the plates. For the irises, the area is $\pi\left(r_{0}^{2}-b^{2}\right)$. This leads to a capacitance $\mathcal{C}$ of

$$
\mathcal{C}=C h=\epsilon \pi\left(r_{0}^{2}-b^{2}\right)
$$

So, we have identified the second term in the denominator of equation (3.55) as $-i \omega \eta \mathcal{C}$.

We can also calculate an inductance for the iris structure from the magnetic flux,

$$
\Phi=L I
$$

where $I$ is the beam current. Using equation (3.44) we calculate the magnetic flux as

$$
\Phi=\int_{0}^{h} \int_{b}^{r_{0}} B_{\theta} d r d z=I \frac{\mu_{0} h}{c_{m}} \ln \left(r_{0} / b\right)
$$

The inductance per length $(\mathcal{L})$ is then

$$
\mathcal{L}=\frac{L}{h}=\frac{\mu_{0}}{2 \pi} \ln \left(r_{0} / b\right)
$$

We can identify the second term in the numerator of equation (3.55) as $-i \omega \mathcal{L}$.

We can re-write the impedance of the iris structure in the low frequency limit using the expressions for the capacitance and inductance

$$
Z=\frac{\eta-i \omega \mathcal{L}}{1-i \omega \eta \mathcal{C}}
$$

Comparing this with the expression for the impedance of a resistor and capacitor in parallel (equation 3.46), we see that the iris structure has the impedance of a resistor 
and capacitor in parallel with an additional part coming from the inductance of the iris structure. If we rationalize equation (3.64),

$$
Z=\frac{\eta+i \omega\left(\eta^{2} \mathcal{C}-\mathcal{L}\right)+\omega^{2} \eta \mathcal{C L}}{1+\omega^{2} \eta^{2} \mathcal{C}^{2}}
$$

then to first order in $\omega$ (which is the order to which we kept terms in the expansion of the Bessel functions) the impedance has the same form as the impedance of a resistor and capacitor in parallel, but with the inductance of the irises acting to decrease the capacitance.

\subsubsection{Connecting the Iris Model to the WARPrz Wall Model}

We make a connection between the electric and magnetic fields at the inner iris radius, $r=b$, that we calculated for the iris structure with the fields calculated in WARPrz. We need to do a transformation because the fields for the iris structure were calculated in the lab frame while the fields in WARPrz are calculated in the beam frame.

In the beam frame, the radial electric field at $r=b$ is given by

$$
E_{r, \text { beamframe }}=\frac{\rho_{0} a^{2}}{2 \epsilon_{0} b}
$$

The surface charge can be related to the charge density by

$$
\sigma=-\rho_{0} \frac{\pi a^{2} l}{2 \pi b l}
$$

So the radial field at the wall in the beam frame is

$$
E_{r, \text { beamframe }}=-\frac{\sigma}{\epsilon_{0}}
$$

We can express the surface charge used in WARPrz (equation (3.16)) in terms of $E_{z}$ by

$$
\sigma=\frac{k}{2 \pi b \eta} \frac{\left(1-i\left(\omega^{\prime}+k v_{b}\right) \eta \mathcal{C}\right)}{\left(\omega^{\prime}+k v_{b}\right)} E_{z}
$$

where the prime on the frequency reminds us that the frequency is the beam frame frequency. Combining these two equations gives the radial electric field from the 
WARPrz model.

$$
E_{r, \text { beamframe }}=-\frac{k}{2 \pi b \eta \epsilon_{0}} \frac{\left(1-i\left(\omega^{\prime}+k v_{b}\right) \eta \mathcal{C}\right)}{\left(\omega^{\prime}+k v_{b}\right)} E_{z}
$$

To compare this field with the iris model, we transform this beam frame electric field into a magnetic field in the lab frame using a Lorentz transform,

$$
B_{\theta, \text { labframe }}=\frac{v_{b}}{c^{2}} E_{r, \text { beamframe }}
$$

where we assume that $\gamma \approx 1$. Substituting the expression for the radial electric field using the frequency in the lab frame and noting that $\omega \approx k v_{b}$, gives

$$
B_{\theta, \text { WARPmodel }}=-\frac{1}{2 \pi b \eta \epsilon_{0}} \frac{1}{c^{2}}(1-i \omega \eta \mathcal{C}) E_{z}
$$

where $E_{z}$ is the same in both the beam and lab frame.

For the iris model, we substitute the expressions for the capacitance and inductance (equations (3.60) and (3.63)) into the expressions for the electric and magnetic fields (equations (3.52) and (3.53)) in the low frequency limit,

$$
\begin{gathered}
\frac{c B_{\theta, \text { iris }}}{K}=-\mu_{0} c \frac{r_{0}}{b}(1-i \omega \eta \mathcal{C}) \\
\frac{E_{z, \text { iris }}}{K}=2 \pi r_{0} \eta(1-i \omega \mathcal{L} / \eta)
\end{gathered}
$$

In order to compare it with the magnetic field in the lab frame from the WARPrz model, we substitute for the surface current $(K)$ from the electric field, use $\mu_{0} \epsilon_{0}=1 / c^{2}$ and assume vacuum between the irises to get,

$$
B_{\theta, \text { iris }}=-\frac{1}{2 \pi b \eta \epsilon_{0}} \frac{1}{c^{2}} \frac{(1-i \omega \eta \mathcal{C})}{(1-i \omega L / \eta)} E_{z}
$$

We then compare the magnetic field in the lab frame due to the wall model in WARPrz (equation (3.72)) with the magnetic field from the iris model (equation (3.75)) and see they are identical if the inductance of the irises is ignored.

In this section, we have proposed a device which is the physical analog of the wall model we use in WARPrz. If the irises are close together, there is no radial electric 
field between the irises which corresponds to no radial electric field outside our wall in WARPrz. The impedance of the iris structure is that of a resistor and capacitor in parallel, like that of the wall in WARPrz, but also includes an inductive component. The inductance comes into the impedance at the same order in frequency as the capacitance does. Equation (3.65) shows that to lowest order in $\omega$ this inductance can be taken into account in WARPrz by reducing the capacitance by an amount $\mathcal{L} / \eta^{2}$. We've also shown the magnetic field due to the iris structure at $r=b$ is the same as the magnetic field which comes about by transforming the radial electric field used in the WARPrz model into a magnetic field in the lab frame. While the wall we are modeling in WARPrz represents the impedance of the accelerating gaps in the induction LINAC driver, the iris model shows that such a wall does not exist only in the computer code, but could be built in the lab.

\subsection{Dispersion Relation for Longitudinal Waves}

Using a cold fluid approximation, the dispersion relation for longitudinal waves on a beam of constant density ions can be calculated without assuming a long wavelength approximation as was done in section 1.3.2. A more general form of this derivation is presented by R. Davidson[8].

Assume we have a cold, cylindrical beam of ions of constant density in a perfectly conducting pipe. The electrostatic potential for this system can be gotten from

$$
\frac{1}{r} \frac{\partial}{\partial r} r \epsilon(r) \frac{\partial \phi}{\partial r}+\frac{\partial}{\partial z} \epsilon(r) \frac{\partial \phi}{\partial z}=0
$$

where

$$
\epsilon=1-\frac{\omega_{p}^{2}}{\omega^{2}}
$$

If we write $\phi(r, z)=\tilde{\phi}(r) \exp (i k z)$, then we get

$$
\frac{1}{r} \frac{\partial}{\partial r} r \epsilon(r) \frac{\partial \tilde{\phi}}{\partial r}-k^{2} \epsilon(r) \tilde{\phi}=0
$$

We notice that $\epsilon(r)$ is constant inside $(r<a)$ and outside $(b<r<a)$ the beam so that it can be pulled out of the derivatives in each region. So, in each region, we need 
to solve

$$
\frac{1}{r} \frac{\partial}{\partial r} r \frac{\partial \tilde{\phi}}{\partial r}-k^{2} \tilde{\phi}=0 .
$$

This equation is a modified Bessel equation, so the solution is

$$
\tilde{\phi}= \begin{cases}A I_{0}(k r), & \text { if } r \leq a, \\ B I_{0}(k r)+C K_{0}(k r), & \text { if } a \leq r \leq b\end{cases}
$$

where we set the coefficient of $K_{0}(k r)$ to zero for $r \leq a$ because it is not finite at the origin.

At the $r=a$ boundary, we match $\tilde{\phi}$ and $\epsilon(r)(\partial \tilde{\phi} / \partial r)$. This gives the conditions

$$
\begin{gathered}
A I_{0}(k a)=B I_{0}(k a)+C K_{0}(k a) \\
\epsilon A I_{1}(k a)=B I_{1}(k a)-C K_{1}(k a)
\end{gathered}
$$

where we use $I_{0}^{\prime}=I_{1}$ and $K_{0}^{\prime}=-K_{1}$. Rearranging these equations gives

$$
\begin{gathered}
\frac{B}{A}=1-(1-\epsilon) k a I_{1}(k a) K_{0}(k a) \\
\frac{C}{A}=(1-\epsilon) k a I_{1}(k a) I_{0}(k a)
\end{gathered}
$$

where we use the relation,

$$
I_{0}(z) K_{1}(z)+I_{1}(z) K_{0}(z)=1 / z
$$

For a perfectly conducting wall, the boundary condition is simply that $\tilde{\phi}=0$ at $r=b$.

$$
0=\frac{B}{A} I_{0}(k b)+\frac{C}{A} K_{0}(k b)
$$

Substituting in the expressions for $B / A$ and $C / A$ gives the dispersion relation

$$
0=1-\frac{\omega_{p}^{2}}{\omega^{2}} k a \frac{I_{1}(k a)}{I_{0}(k b)}\left(K_{0}(k a) I_{0}(k b)-I_{0}(k a) K_{0}(k b)\right)
$$




\subsubsection{Long Wavelength Limit}

The dispersion relation must reproduce the results of the long wavelength approximation calculation. Since $k=2 \pi / \lambda$, long wavelength means small $k$. In the limit that $k a$ and $k b$ are much smaller than 1, we can expand the Bessel functions using their small argument approximations.

$$
\begin{gathered}
I_{0}(x) \simeq 1 \\
I_{0}^{\prime}(x)=I_{1}(x) \simeq x / 2 \\
K_{0}(x) \simeq-\ln (x / 2) \\
K_{0}^{\prime}(x)=-K_{1}(x) \simeq-1 / x
\end{gathered}
$$

Using these expansions in the dispersion relation yields

$$
0=1-\frac{\omega_{p}^{2}}{\omega^{2}} \frac{(k a)^{2}}{2} \ln (b / a)
$$

When the density is constant, we can relate the line charge density to the charge density by

$$
\rho_{0}=\frac{\lambda_{0}}{\pi a^{2}}
$$

So, the plasma frequency can be replaced by

$$
\omega_{p}^{2}=\frac{\rho_{0} e}{M \epsilon_{0}}=\frac{\lambda_{0}}{\pi a^{2}} \frac{e}{M \epsilon_{0}}
$$

Plugging this in to the dispersion relation and rearranging gives

$$
\omega^{2}=k^{2} \frac{e \lambda_{0}}{M} \frac{\ln (b / a)}{2 \pi \epsilon_{0}}=k^{2} v_{p}^{2}
$$

which is the dispersion relation that we get in the long wavelength limit when $\eta=0$.

\subsection{Dispersion Relation Including Capacitive and Resistive Wall}

We extend the dispersion relation of the previous section to include a wall with resistance and capacitance as in the RZ thread of the WARP code. The wall is a 
boundary condition on the problem. The solution inside the pipe is the same as in the previous section

$$
\tilde{\phi}= \begin{cases}A I_{0}(k r), & \text { if } r \leq a, \\ B I_{0}(k r)+C K_{0}(k r), & \text { if } a \leq r \leq b .\end{cases}
$$

We still match $\tilde{\phi}$ and $\epsilon(r)(\partial \tilde{\phi} / \partial r)$ at $r=a$ to give

$$
\begin{gathered}
\frac{B}{A}=1-(1-\epsilon) k a I_{1}(k a) K_{0}(k a) \\
\frac{C}{A}=(1-\epsilon) k a I_{1}(k a) I_{0}(k a)
\end{gathered}
$$

and $\epsilon=1-\omega_{p}^{2} / \omega^{2}$.

At the wall, we have a surface charge. In WARPrz, we advance the surface charge using

$$
\frac{\partial \sigma}{\partial t}=\frac{1}{2 \pi b} \frac{\partial}{\partial z}\left[\frac{1}{\eta}\left(\frac{\partial \phi}{\partial z}+\eta \mathcal{C} \frac{\partial}{\partial t} \frac{\partial \phi}{\partial z}\right)\right]
$$

and then we advect the surface charge and the potential at the old time (used in the time derivative on the right hand side) since our calculation is done in the beam frame. We can add this advection to the above equation by noting that in the beam

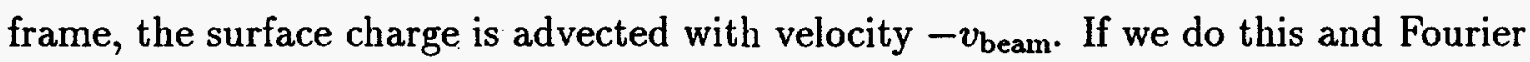
transform with respect to $t$ and $z$ we get

$$
\sigma=\frac{-i k^{2}}{2 \pi b \eta} \frac{\left(1-i \eta \mathcal{C}\left(\omega+k v_{b}\right)\right)}{\left(\omega+k v_{b}\right)} \tilde{\phi}
$$

The boundary condition is given by

$$
\left(\mathbf{D}_{2}-\mathbf{D}_{1}\right) \cdot \hat{n}=\sigma
$$

where $\mathbf{D}_{2}$ is the field just outside the pipe and $\mathbf{D}_{1}$ is the field just inside the pipe. In our case, we assume the radial electric field outside of the pipe is zero, so we are left with

$$
-\epsilon_{0} E_{r}(r=b)=\sigma
$$

Using our expression for $\phi, B / A$, and $C / A$ gives

$$
\begin{gathered}
I_{0}(k b)+\frac{\omega_{p}^{2}}{\omega^{2}} k a I_{0}^{\prime}(k a)\left[I_{0}(k a) K_{0}(k b)-K_{0}(k a) I_{0}(k b)\right]= \\
\frac{i 2 \pi b \eta \epsilon_{0}}{k} \frac{\left(\omega+k v_{b}\right)}{\left(1-i \eta \mathcal{C}\left(\omega+k v_{b}\right)\right)}\left\{I_{0}^{\prime}(k b)+\frac{\omega_{p}^{2}}{\omega^{2}} k a I_{0}^{\prime}(k a)\left[I_{0}(k a) K_{0}^{\prime}(k b)-K_{0}(k a) I_{0}^{\prime}(k b)\right]\right\}
\end{gathered}
$$




\subsubsection{Long Wavelength Limit}

In the long wavelength limit, $k a, k b<<1$ and we use the small argument expansions for the Bessel functions.

$$
\begin{gathered}
I_{0}(x) \simeq 1 \\
I_{0}^{\prime}(x)=I_{1}(x) \simeq x / 2 \\
K_{0}(x) \simeq-\ln (x / 2) \\
K_{0}^{\prime}(x)=-K_{1}(x) \simeq-1 / x
\end{gathered}
$$

We also define $v_{p}^{2}=Z e \lambda_{0} g / M$ and $g=\ln (b / a) / 2 \pi \epsilon_{0}$. Using these in our dispersion relation gives

$$
1-\frac{k^{2} v_{p}^{2}}{\omega^{2}}=i \eta \frac{\left(\omega+k v_{b}\right)}{\left(1-i \eta \mathcal{C}\left(\omega+k v_{b}\right)\right)}\left[-\frac{v_{p}^{2}}{g \omega^{2}}\left(1-\frac{k^{2} b^{2}}{2} \ln \frac{k a}{2}\right)+\pi b^{2} \epsilon_{0}\right]
$$

Next, we examine the magnitude of these terms. We compare

$$
\left|\frac{k^{2} b^{2}}{2} \ln \frac{k a}{2}\right|=\left|\frac{k^{2} b^{2}}{2} \ln \frac{f k b}{2}\right|
$$

with 1 for $k a, k b$ small, where we define the fraction of the pipe filled by beam, $f=a / b$. In the limit that $k b \rightarrow 0$,

$$
\lim _{k b \rightarrow 0}\left|\frac{k^{2} b^{2}}{2} \ln \frac{f k b}{2}\right|=0
$$

For $k b$ small but not zero, we show that

$$
\left|\frac{k^{2} b^{2}}{2} \ln \frac{j k b}{2}\right|<1
$$

by plotting as a function of $k b$ and $f$. Figure (3.10) shows this quantity for values of $k b$ ranging from .001 to .2 and fill fraction, $f$ ranging from .1 to .9 . Over this range of parameters

$$
\left|\frac{k^{2} b^{2}}{2} \ln \frac{f k b}{2}\right|<.1
$$

and we can neglect that term compared with the 1 . 


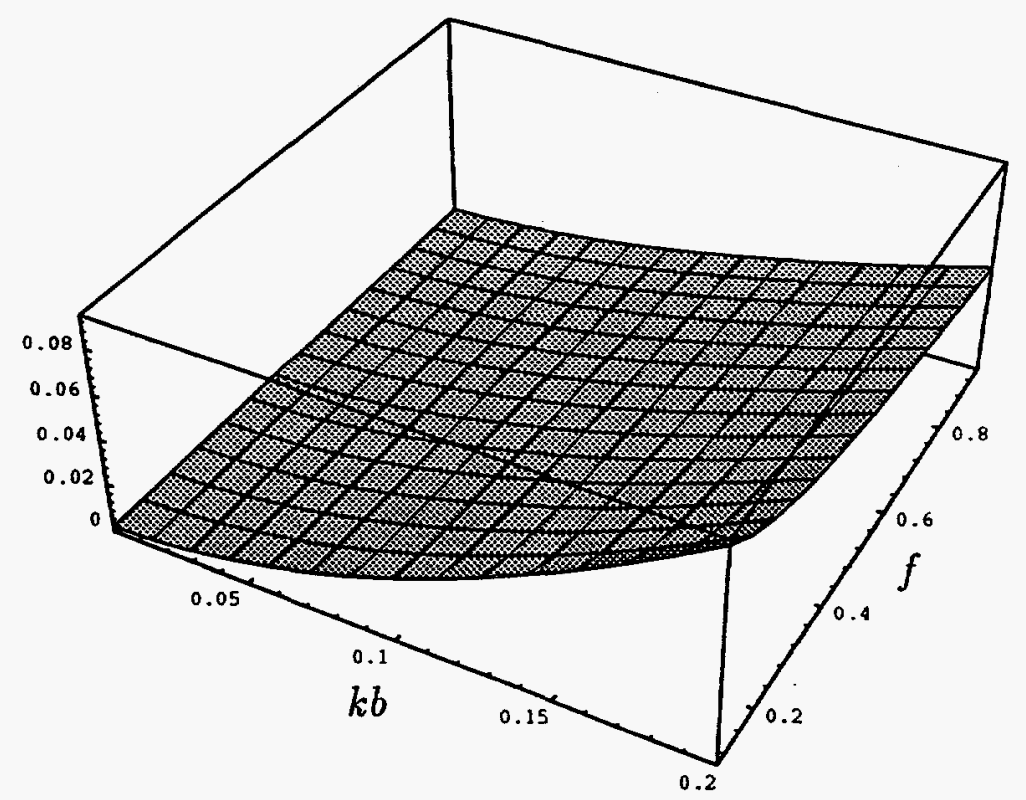

Figure 3.10: A plot of $\left|(k b)^{2} / 2 \ln (f k b / 2)\right|$ as a function of $k b$ and the fraction of the pipe which is filled, $f$, shows that this quantity is smaller than 1 for $k b$ less than 2

Since $\omega^{2} \approx k^{2} v_{p}^{2}$ and $g=\ln (b / a) / 2 \pi \epsilon_{0}$, we can write

$$
\frac{v_{p}^{2}}{g \omega^{2}} \approx \pi b^{2} \epsilon_{0} \frac{2}{(k b)^{2} \ln (b / a)}>>\pi b^{2} \epsilon_{0}
$$

so that we can neglect the $\pi b^{2} \epsilon_{0}$ term relative to the $v_{p}^{2} / g \omega^{2}$ term. This leaves us with

$$
1-\frac{k^{2} v_{p}^{2}}{\omega^{2}}=i \eta \frac{\left(\omega+k v_{b}\right)}{\left(1-i \eta \mathcal{C}\left(\omega+k v_{b}\right)\right)}\left[-\frac{v_{p}^{2}}{g \omega^{2}}\right]
$$

which is the dispersion relation we got in section 1.3 by using the 1-d long wavelength approximation to the problem.

\subsection{Testing This Model in WARPrz}

We wanted our particle code tests to be in the regime where the linear, cold beam, fluid theory is valid. As a result, we wanted a cold beam so that the results would 
not be influenced by thermal effects which were neglected in the fluid theory and we wanted to use very small perturbations so the results would not be influenced by any nonlinear effects which were also not included in the fluid theory.

In order to use very small perturbations, we need to load the particles such that there is as little noise in the calculation as possible. Any significant noise will mask the very small perturbations that have been excited on the beam. Any time a particle moves from one cell to the next, we can get noise since this can cause an imbalance in the number of particles in each cell (cell $a$ now has 11 particles while cell $b$ has 9). One way to reduce noise is to use large numbers of particles. However, to get the low levels of noise that we want, we would have to use a very large number of particles which would make the simulations very long and costly.

Instead of using a very large number of particles to reduce the noise level, we use a clever technique. If we give each particle a very large mass, then it will never move very far. If we can keep particles from moving across cell boundaries, then the noise level will be very low.

For our tests, we want to load the particles such that the charge density is a constant. Implementing such a scheme in a cartesian coordinate system is easy. We simply put one particle in the center of each cell. In a cylindrical system, things get more complicated because the volume of each radial cell is larger than the last one if we use a constant $\Delta r$. This introduces two problems:

1. The charge density at each gridpoint is determined by dividing the fraction of the charge assigned to that gridpoint by the volume at that gridpoint. If we put one particle in the center of a cell, it will contribute the same amount of charge to the gridpoint at $r_{i}$ as it does to the gridpoint $r_{i+1}$, but the charge density will be smaller at $r_{i+1}$ since the volume is larger there. This tells us that we don't want to put the particles at the center of the cells, but instead we want to move them slightly closer to the gridpoint at the larger radius.

2. Because the volume of a cell increases with radius, we need to put more particles in the cells with larger radius than those with smaller radius in order to get a 
constant density if all particles have the same weighting. In the WARP code, we have chosen to give the particles constant weighting.

\subsubsection{Particle Loading for Linear, Cold Beam Test Runs}

We need to load the particles such that the charge density, $\rho$, is a constant. This gives us a linear radial electric field, $E_{\tau} \propto r$. Since we are in cylindrical coordinates, each radial cell has a larger volume than the last. To compensate for this, we need more particles in each cell as we go away from the origin. The particles also have to be moved from the cell center in the radial direction to compensate for the different volume at the two gridpoints adjoining a cell.

First, we show that a constant charge density gives a linear radial electric field in our difference equations. Recall equations (2.19) and (2.20) from section 2.2.3

$$
\begin{aligned}
\frac{Q_{j, k}}{\epsilon_{0}}= & 2 \pi \Delta z\left(r_{j+1 / 2} E_{r, j+1 / 2, k}-r_{j-1 / 2} E_{r, j-1 / 2, k}\right)+ \\
& \pi\left(r_{j+1 / 2}^{2}-r_{j-1 / 2}^{2}\right)\left(E_{z, j, k+1 / 2}-E_{z, j, k-1 / 2}\right)
\end{aligned}
$$

for $j \neq 0$ and

$$
\frac{Q_{0, k}}{\epsilon_{0}}=2 \pi r_{1 / 2} \Delta z E_{\tau, 1 / 2, k}+\pi r_{1 / 2}^{2}\left(E_{z, 0, k+1 / 2}-E_{z, 0, k-1 / 2}\right)
$$

where $r_{j+1 / 2}$ is the radius half way between the gridpoints, $r_{j+1 / 2}=1 / 2\left(r_{j+1}+r_{j}\right)$. The charge density is defined as $\rho_{j, k}=Q_{j, k} / V_{j, k}$ where the volume is $V_{j, k}=\pi\left(\Delta r^{2}\right)_{j} \Delta z$ with $\left(\Delta r^{2}\right)_{j}=r_{j+1 / 2}^{2}-r_{j-1 / 2}^{2}$ for $j>0$ and $\left(\Delta r^{2}\right)_{0}=r_{1 / 2}^{2}$ for $j=0$. For $E_{z}=0$ and $E_{r, j+1 / 2} \propto r_{j+1 / 2}$, these equations simplify to

$$
\frac{Q_{j}}{\epsilon_{0}} \propto 2 \pi \Delta z\left(r_{j+1 / 2}^{2}-r_{j-1 / 2}^{2}\right)
$$

for $j \neq 0$ and

$$
\frac{Q_{0}}{\epsilon_{0}} \propto 2 \pi \Delta z r_{1 / 2}^{2}
$$

for $j=0$. The charge density is then

$$
\rho_{j}=\frac{Q_{j}}{V_{j}} \propto 2 \pi \epsilon_{0} \Delta z \frac{\left(r_{j+1 / 2}^{2}-r_{j-1 / 2}^{2}\right)}{\pi \Delta z\left(r_{j+1 / 2}^{2}-r_{j-1 / 2}^{2}\right)}=\text { constant }
$$


for $j \neq 0$ and

$$
\rho_{0}=Q_{0} / V_{0} \propto \frac{2 \pi \epsilon_{0} \Delta z r_{1 / 2}^{2}}{r_{1 / 2}^{2}}=\text { constant }
$$

If we load a beam which has constant charge density at each gridpoint, then our difference scheme for the Poisson solve will produce a linear radial electric field.

Because the cell volume increases as we go away from the origin in a cylindrical coordinate system, we need to put multiple particles in each cell to give a constant charge density. This is necessary because we have chosen to give the particles equal weights (i.e. each simulation particle represents the same number of real particles). Given a constant density, $\rho_{0}$, then the charge at a gridpoint is given by $Q_{i}=\rho_{0} V_{i}$, where $V_{i}$ is the volume. This gives

$$
Q_{i}= \begin{cases}\left(2 \pi \rho_{0} \Delta r \Delta z\right) \frac{\Delta r}{8}, & \text { if } i=0 \\ \left(2 \pi \rho_{0} \Delta r \Delta z\right) i \Delta r, & \text { if } i>0\end{cases}
$$

This tells us that if we have one unit of charge at $i=1$, then we want two units of charge at $i=2$ and so on. However, one unit of charge at $i=1$ would give us $1 / 8$ unit of charge at the origin. We can easily fix this by having one charge at 0 , eight charges at 1,16 charges at 2 , and $8 i$ charges at gridpoint $i$. These charges are distributed such that one half the charge at a gridpoint comes from the cell to the left and one half of the charge comes from the cell to the right. If we number the radial cells by the smaller of the gridpoints bounding it, the zeroth cell has 5 particles in it ( 1 from $i=0$ and 4 from $i=1$ ), the first cell has 12 particles, the second cell has 20 particles, and the $i$-th cell has $8 i+4$ particles in it.

Once we know how many particles go in each cell, we need to determine where they are placed within the cell. Placing the particles at the center of the cell would not give equal charge density at the gridpoints surrounding the cell because the volume is larger at the larger radius. First consider a single cell which is not at the origin. The inner boundary of this cell will be taken to be at $r_{i}$ and the outer boundary of the cell at $r_{i+1}=r_{i}+d r$. The charge density for gridpoint $i$ is given by

$$
\rho_{i}=\frac{Q_{i}}{2 \pi r_{i} \Delta r \Delta z}
$$


where $Q$ is the charge that is distributed to the gridpoint from the particles. We place a particle in the center of the cell with respect to $z$ and a distance $D$ from $r=r_{i}$ in the radial direction. Using linear weighting, the charge for the two different radii is then given by

$$
Q_{i}=\frac{q}{\Delta r \Delta z} \frac{\Delta z}{2}(\Delta r-D)
$$

and

$$
Q_{i+1}=\frac{q}{\Delta r \Delta z} \frac{\Delta z}{2} D
$$

The charge densities are then

$$
\rho_{i}=\frac{q}{2 \Delta r} \frac{\Delta r-D}{2 \pi r \Delta r \Delta z}
$$

and

$$
\rho_{i+1}=\frac{q}{2 \Delta r} \frac{D}{2 \pi(r+\Delta r) \Delta r \Delta z} .
$$

We set these densities equal to each other and solve for $D$ to get

$$
D=\frac{r+\Delta r}{2 r+\Delta r} \Delta r
$$

This tells us where to place the particles radially within each cell. In the first cell, the particles are placed at $r=(4 / 5) \Delta r$ so the charge density at the 0th gridpoint is twice that of the 1st gridpoint. This is necessary because the 1st gridpoint also receives charge from the next cell while the 0th gridpoint does not.

This scheme works up to the last cell. Call the last occupied cell the $i a$-th cell. This means that we have nonzero density for gridpoints $i a$ and $i a+1$, and zero density beyond that. If we put $8 i a+4$ particles in the last occupied cell, we will have one half the required density at the very last gridpoint $(i a+1)$ since it will not receive a contribution from the cell beyond it. We can fix this by increasing the number of particles in the last cell and moving them such that the density is constant out to the last occupied gridpoint. If we do this, we find that we need $12 i a+8$ particles in the last cell and they should be located at $D=(8 i a+8) /(12 i a+8)$.

So, if we wish to place particles in cells 0 through $i a$, we do the following. In the zeroth cell, put 5 particles at $r=4 / 5 \Delta r$ 
In cells 1 through $(i a-1)$, put $8 i+4$ particles at

$$
r=i \Delta r+\frac{i+1}{2 i+1} \Delta r
$$

In the last cell, ia, put $12 i a+8$ particles at

$$
r=i a \Delta r+\frac{8 i a+8}{12 i a+8} \Delta r
$$

We can get the total number of particles in one axial slice by doing the sum.

$$
N_{p, \text { slice }}=5+\sum_{i=1}^{i a-1}(8 i+4)+12 i a+8
$$

and the total number of particles in $i z$ axial slices is then

$$
N_{p}=i z(4(i a+1)(i a+2)+1)
$$

\subsubsection{Results using Linear Particle Loading}

We ran the code with this linear particle loading so that we could compare our 2-d particle code with the linear cold beam theory. In each of these runs, we made the mass of the particles very large $\left(M=1 \times 10^{10} \mathrm{amu}\right)$ so that we would observe only linear behavior. We compensate for the large mass by making the beam current very large. This keeps the macroscopic quantities such as the wave speed, growth rate, and plasma frequency all realistic. The special loading gives us a very quiet run for very small perturbations. These beams were periodic and infinite in length.

We gave the beams a sinusoidal perturbation in the velocity. Since our model assumes constant density, we used the condition $\nabla \cdot \mathbf{u}=0$ to determine our two components of velocity, $v_{r}$ and $v_{z}$. In these runs, these velocities were given by

$$
\begin{gathered}
v_{z}=v_{\text {beam }}+\tilde{v} \cos (k z) \\
v_{r}=\tilde{v} \frac{r k}{2} \sin (k z)
\end{gathered}
$$

where $\tilde{v}$ is the size of the perturbation and is generally $0.0001 v_{p}$. This causes a perturbation in line charge density to grow. We did not attempt to separate the 
forward traveling wave from the backward traveling wave. Since the forward traveling wave decays with resistance, we just allow it to decay and after a few $\boldsymbol{e}$-folding times are left with a pure backward traveling wave.

In each case, we made the resistance unrealistically large. This was done to speed up the runs by making the growth rate larger than it will be in a heavy ion fusion driver.

The cases that we ran are:

\section{Long wavelength with resistance only}

This run had a resistance of $900 \mathrm{ohms} / \mathrm{m}$ and no capacitance. The wave number times the pipe radius $(k b)$ was .031 which is in the long wavelength limit. The code gave a growth rate of $6.25 \times 10^{5} \mathrm{~s}^{-1}$ which compares with the theoretical growth rate of $6.169 \times 10^{5} \mathrm{~s}^{-1}$ for an error of $1.3 \%$.

\section{Long wavelength with resistance and capacitance}

This run had a resistance of $900 \mathrm{ohms} / \mathrm{m}$ and an $\mathrm{RC}$ time of $1.25 \times 10^{-6}$ seconds. This run also had $k b=.031$ so that it was in the long wavelength regime. The code gave a growth rate of $1.11 \times 10^{4} \mathrm{~s}^{-1}$ and the theory gave a growth rate of $1.093 \times 10^{4} \mathrm{~s}^{-1}$ which is an error of $1.5 \%$.

\section{Short wavelength perturbation with resistance only}

Since the growth rate falls of sharply as the wavelength gets shorter, we increased the resistance to decrease the run time. In this case, the resistance was $7200 \mathrm{ohms} / \mathrm{m}$ and no capacitance. This run had $k b=1.26$ which is well out of the long wavelength regime. The code gave a growth rate of $3.06 \times 10^{6} \mathrm{~s}^{-1}$ and the theory gave a growth rate of $3.047 \times 10^{6} s^{-1}$. The long wavelength theory (which should not be applicable in this case) gave a growth rate of $4.870 \times 10^{6} \mathrm{~s}^{-1}$. 
Short wavelength perturbation with resistance and capacitance

Since capacitance decreases the growth rate, we had to increase the resistance even further for this run. In this run the resistance was $5.76 \times 10^{4} \mathrm{ohms} / \mathrm{m}$ and the $\mathrm{RC}$ time was $1.0 \times 10^{-7}$ seconds. This run had $k b=1.26$. The code gave a growth rate of $3.62 \times 10^{5} \mathrm{~s}^{-1}$ and the theory gave a growth rate of $3.670 \times 10^{5} \mathrm{~s}-1$. The long wavelength theory (which should not be applicable in this case) gave a growth rate of $1.249 \times 10^{6} \mathrm{~s}^{-1}$. 


\section{Chapter 4}

\section{Simulations with Module Impedance}

After verifying that our model agreed with cold beam fluid theory when tested with a cold infinite length beam with a sinusoidal perturbation, we moved on to more realistic simulations. Our first simulations of the longitudinal instability were to study a single perturbation launched from the beam head. By doing this, we can not only watch the perturbation grow as it travels the length of the beam but also see what happens when the perturbation reaches the beam end.

Since we currently have the capability to study drifting (not accelerating) beams in WARPrz, parameters for the simulation were chosen to be those near the end of a proposed driver-scale accelerator where the growth rate of the instability is the largest. In a driver, the beam will not spend much time in this parameter regime and so these simulations are a worst case. These parameters are shown in Table 1. 


\begin{tabular}{|l|r|}
\hline Beam velocity & $c / 3$ \\
\hline Beam current & 3000 Amps \\
\hline Pulse length & 10 meters \\
\hline Beam radius/Pipe radius & .4 \\
\hline Perpendicular temperature & $10 \mathrm{keV}$ \\
\hline Parallel temperature & $10 \mathrm{keV}$ \\
\hline
\end{tabular}

Table 4.1: Beam parameters used in WARPrz simulation

\subsection{Beam Temperature}

We showed in the introduction that $\Delta p / p=.1 \%$ leads to a maximum longitudinal beam temperature of $10 \mathrm{keV}$ because

$$
T_{z}=\frac{\Delta p^{2}}{2 M}=\left(\frac{\Delta p}{p}\right)^{2} \frac{p^{2}}{2 M}=10 \mathrm{keV}
$$

for $p^{2} / 2 M=10 \mathrm{GeV}$ at the end of the accelerator.

In the perpendicular direction, there is a maximum beam emittance for focusing of $\epsilon=3 \times 10^{-5}$ meter-radians. This leads to a perpendicular temperature

$$
T_{\perp}=\frac{\left\langle p_{\perp}^{2}\right\rangle}{2 M}=\frac{p^{2} \sin ^{2} \theta}{2 M} \approx \frac{p^{2} \theta^{2}}{M}
$$

where the angle $\theta$ is assumed to be small. This angle is approximately given by $\theta=\epsilon / a$ where $a$ is the beam radius and $\epsilon$ is the emittance. For a beam with $\epsilon=3 \times 10^{-5}$ meter-radians, $a=.03$ meters, and energy $10 \mathrm{GeV}$, the perpendicular temperature is again $10 \mathrm{keV}$.

At the end of the accelerator, the maximum beam temperature we can focus on target is $10 \mathrm{keV}$ in both the longitudinal and perpendicular directions. In most of the simulations in this thesis, the beam temperature is set to the maximum temperature in both directions. The temperatures are kept equal because of a rapid exchange of energy from the perpendicular direction to the longitudinal direction seen in simulations. The temperature is set to $10 \mathrm{keV}$ (the maximum temperature) to reduce the numerical heating in the simulations while keeping $\Delta z$ and $\Delta r$ reasonably large. In simulations of cooler beams, the Debye length is smaller and more cells are needed to 
keep the ratio of the grid spacing to the Debye length from getting too large. Using a very large number of cells makes the simulations very long and time consuming.

\subsection{Simulations with a Purely Resistive Wall}

The presence of the resistive wall causes the beam to lose energy and slow down. Since we want the beam to travel at the same constant speed as the window in which field calculations are done, we add an external accelerating field which just compensates for the loss to the resistive wall. For simulations in which the wall impedance is purely resistive, the external electric field applied was simply,

$$
E_{z, \text { external }}(r, z)=\eta I_{0}(z)
$$

where $I_{0}(z)$ is the initial current profile as a function of $\mathrm{z}$. Since the axial electric field does not vary much across the beam radius, we applied the same external field at all radii. We found that this field was slightly in error in the beam ends. This caused perturbations of roughly gaussian shape to be launched from the beam head and tail. We used these perturbations as the seed for the instability.

\subsubsection{Large Resistance Simulation $(\eta=200 \Omega / \mathrm{m})$}

Figure (4.1) shows three snapshots of the electrostatic potential on axis $v s z$ and $z$ $v_{z}$ phase space for a simulation with $200 \Omega / \mathrm{m}$ resistance. This is about twice the resistance expected in a driver and shows how the instability can degrade the beam. The perturbation launched from the beam head (right hand side of the plots) moves towards the beam tail and grows. As the perturbation becomes large, the trailing edge steepens as it enters a nonlinear regime.

A similar perturbation is launched from the beam tail, but it decays as it travels forward.

By $17.5 \mu$ s (Figure $(4.1 \mathrm{c})$ ), some particles have gone off the scale in the $z-v_{z}$ phase space plot. These particles will be lost in the accelerator since the confining 
a)

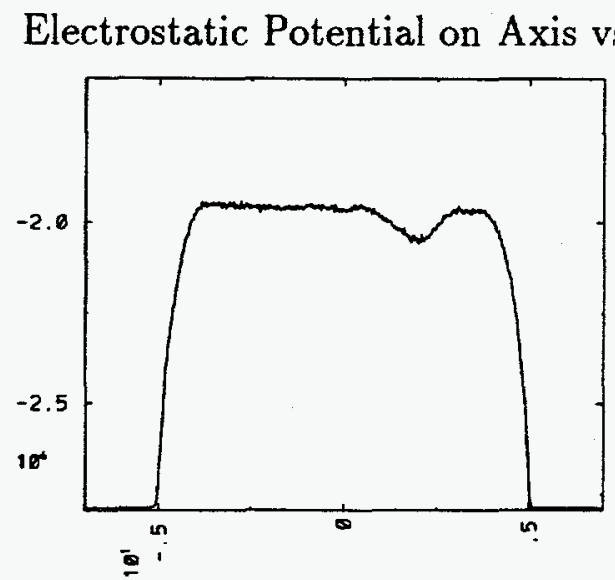

Electrostatic Potential on Axis vs $z$

b)

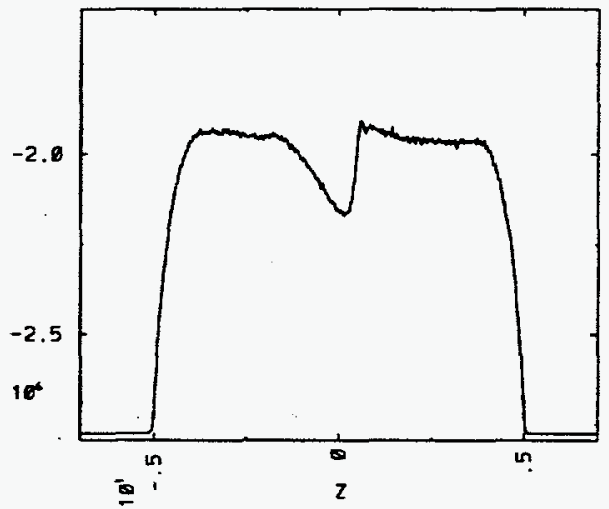

Electrostatic Potential on Axis vs $z$

c)
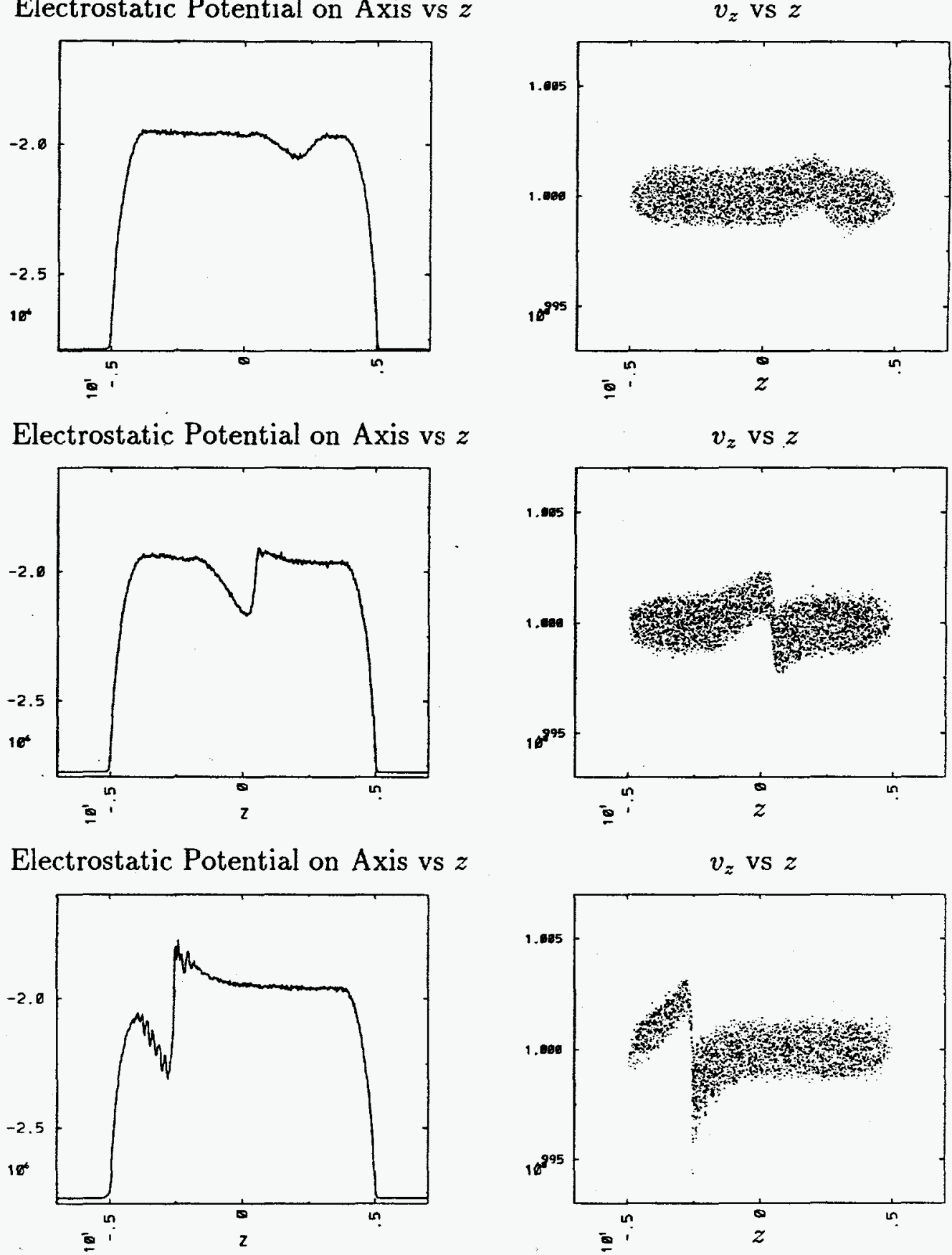

$v_{z}$ Vs $z$

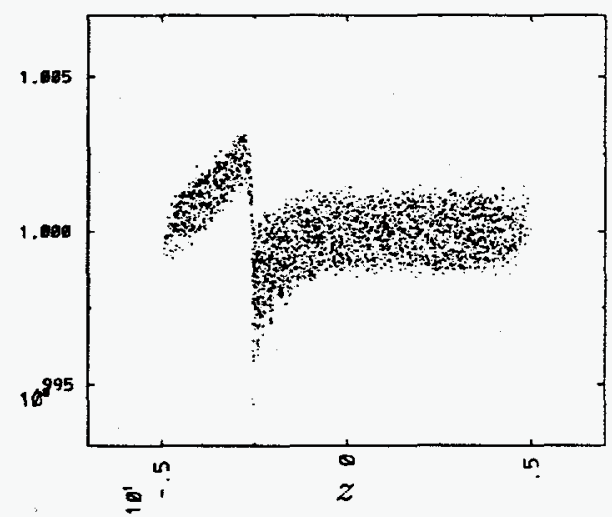

Figure 4.1: A simulation with $200 \Omega / \mathrm{m}$ resistance shows large amounts of growth. (a) $6.6 \mu \mathrm{s}$, (b) $10.9 \mu \mathrm{s}$, (c) $17.5 \mu \mathrm{s}$ 
ear fields will not be large enough to reflect them. In these simulations, the axial confining fields extend over the entire simulation window. As a result, all particles are eventually reflected off the ear fields and no particles are lost. In an accelerator, any particle which gains very large longitudinal velocity will be lost.

This simulation shows how the instability can cause major problems because large numbers of particles can be lost. When perturbations become large, particles can end up with axial velocities much different than the bulk of the beam. When these particles hit the end of the beam, they are either lost because the ear field is not large enough to turn them around, or they cause a larger spread of longitudinal velocities if they are reflected. A large spread of longitudinal velocities is not acceptable for the focusing system.

Large perturbations can also cause problems in more subtle ways. The beam tends to remain incompressible (or near incompressible) as the perturbation moves by. This means that the line charge density perturbation is made up mostly of a perturbation in the beam radius. This puts particles at larger radii, closer to the focusing elements. At these large radii, the focusing fields have larger higher order fields which can cause momentum spread of the particles. The focusing field in WARPrz is linear with radius and constant with $z$, so that such effects are not modeled.

\subsubsection{More Moderate, More Realistic Resistance Simula- tion $(\eta=100 \Omega / \mathrm{m})$}

Figure (4.2) shows the same plots for a wall resistance of $100 \Omega / \mathrm{m}$. This is the resistance expected in a driver. Again a perturbation is launched from the beam head. In this case, the growth is more moderate and the perturbation never becomes large enough to exhibit the extreme behavior seen in the previous case. The perturbation remains roughly gaussian in shape but grows as it travels the length of the beam. Measurement of the growth rate shows that it is about $15 \%$ smaller than the cold beam theory results would predict. This will be discussed in more detail in chapter 5. 
a)

Electrostatic Potential on Axis vs $z$

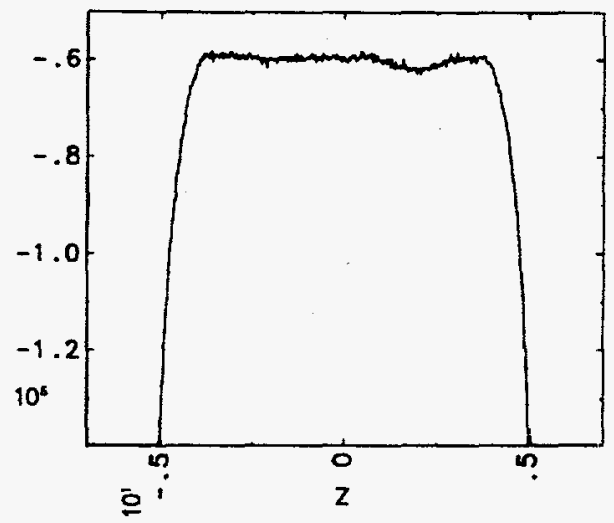

Electrostatic Potential on Axis vs $z$

b)

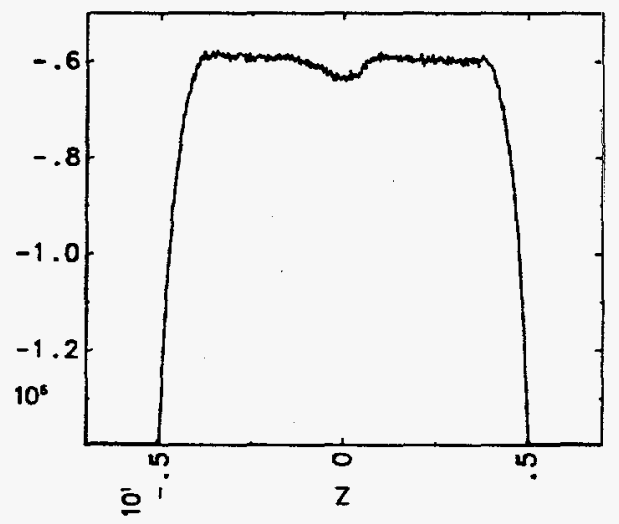

Electrostatic Potential on Axis vs $z$

c)

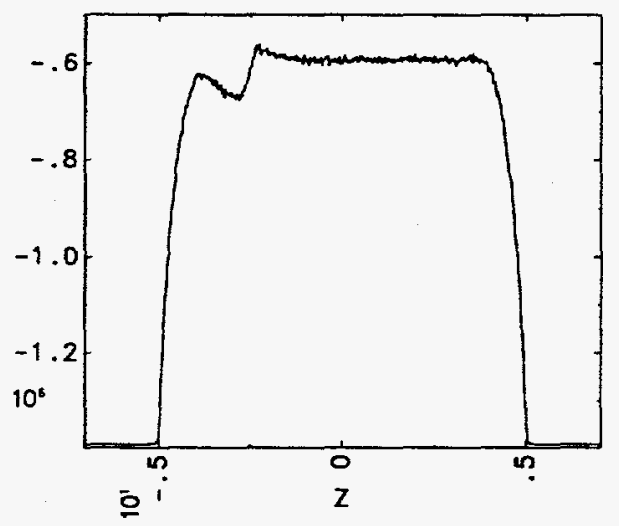

$v_{z}$ VS $z$

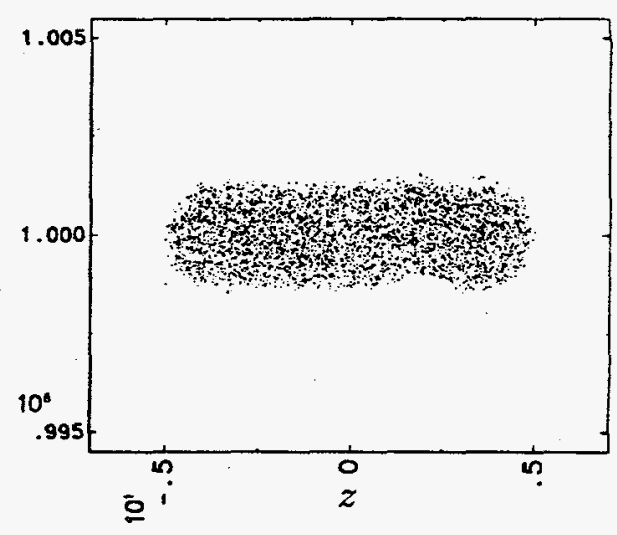

$v_{z}$ vs $z$

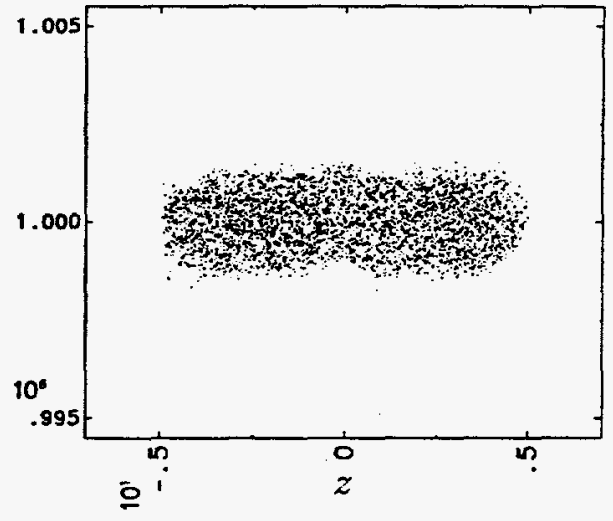

$v_{z}$ Vs $z$

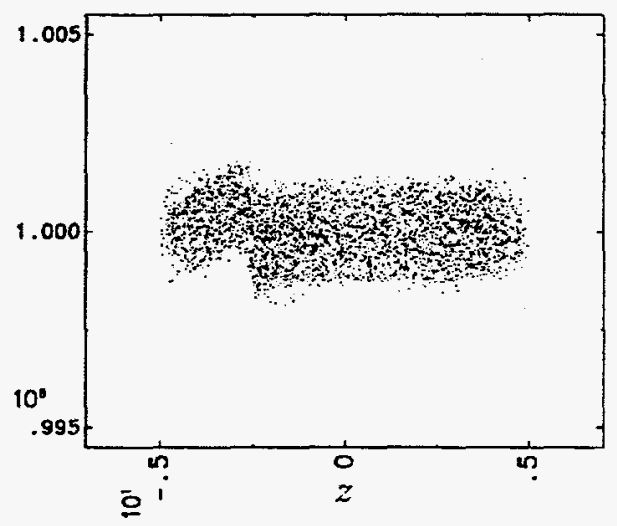

Figure 4.2: A simulation with $100 \Omega / \mathrm{m}$ resistance shows moderate growth. (a) 6.6 $\mu \mathrm{s}$, (b) $10.9 \mu \mathrm{s}$, (c) $17.5 \mu \mathrm{s}$ 
The perturbation slows down when it enters the beam "end" because the line charge density becomes small and thus, the wave velocity (which is proportional to the line charge density) becomes small. It reaches the end of the pulse, reflects off the end, and becomes a forward traveling wave. For a small sinusoidal perturbation, continuity equation for line charge density gives

$$
\frac{\tilde{\lambda}}{\lambda_{0}}=\frac{\tilde{u}}{(\omega / k)}
$$

So for a backward wave $\left(\omega / k=-v_{p}\right)$, the line charge density perturbation and the velocity perturbation have opposite sign, while those quantities have the same sign for a forward wave $\left(\omega / k=v_{p}\right)$. From this, we expect to see the sign of one of these quantities flip when the perturbation reflects off the beam end. Figure (4.3) shows that in this case, the line charge density (which has the same profile as the electrostatic potential on axis) has changed sign. Whether the line charge density perturbation or the velocity perturbation will be the one to change sign seems to depend on the exact details of the beam profile in the end.

During the reflection, the perturbation tends to steepen and become narrower. This can be seen by comparing figure ( $4.2 \mathrm{c}$ ) which shows the perturbation just before reflection, with figure $(4.3 \mathrm{a})$ which shows the perturbation just after reflection. This is believed to be a nonlinear effect, as the larger perturbation is as it enters the beam end, the narrower it becomes. This same effect has been seen in 1-d particle calculations[6]. As the line charge density falls off in the beam end, the perturbation becomes a larger fraction of the "background" or zero-order line charge density. This makes the perturbation look very large when it is in the beam end and nonlinear effects can occur.

The narrowing of the perturbation can cause growth on a complete round trip in some regimes. For a single wavelength, the growth and decay rates are the same. Thus, a complete round trip results in no overall growth because the perturbation grows by $\exp \left(\gamma_{1} \tau\right)$ from head to tail, reflects, then decays by $\exp \left(-\gamma_{1} \tau\right)$. The net effect is no growth. However, if the perturbation narrows and thus changes "wavelength," the decay rate can be smaller than the growth rate for some wavelengths. When this 
Electrostatic Potential on Axis vs $z$

a)

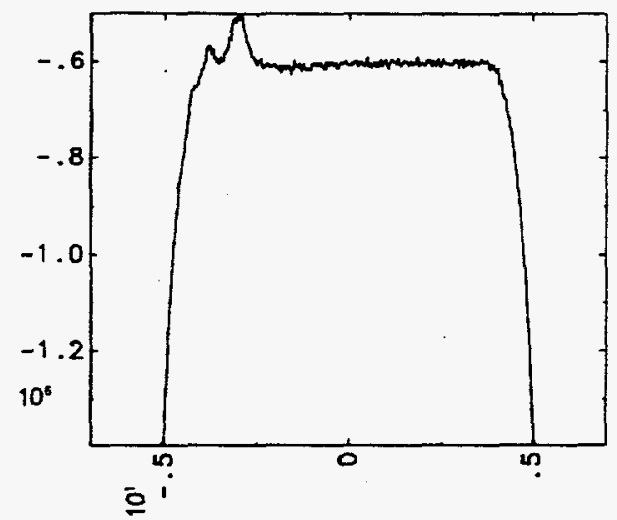

Electrostatic Potential on Axis vs $z$

b)

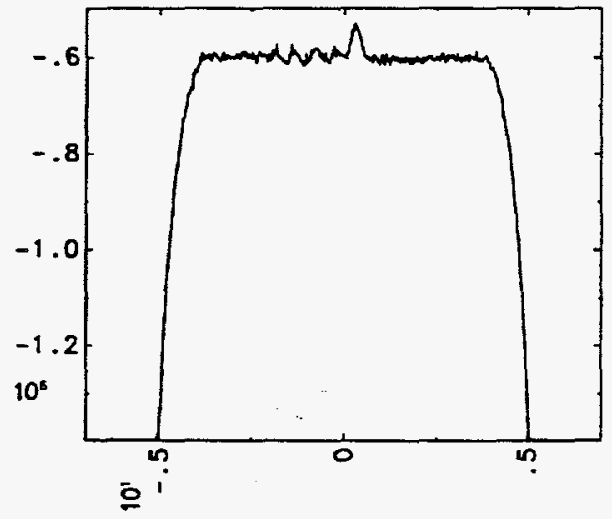

Electrostatic Potential on Axis vs $z$

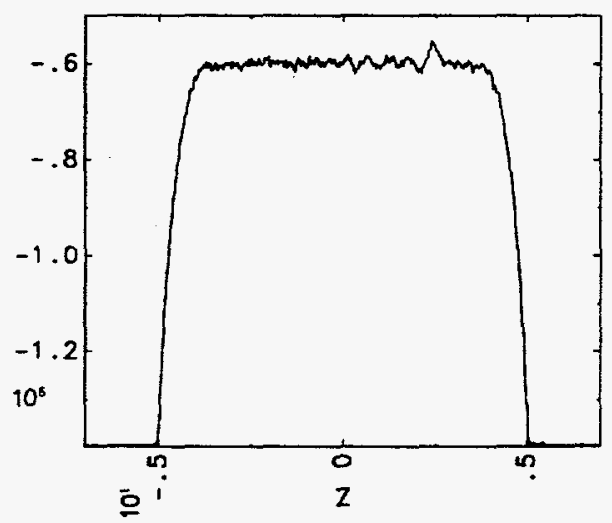

$v_{z}$ vs $z$

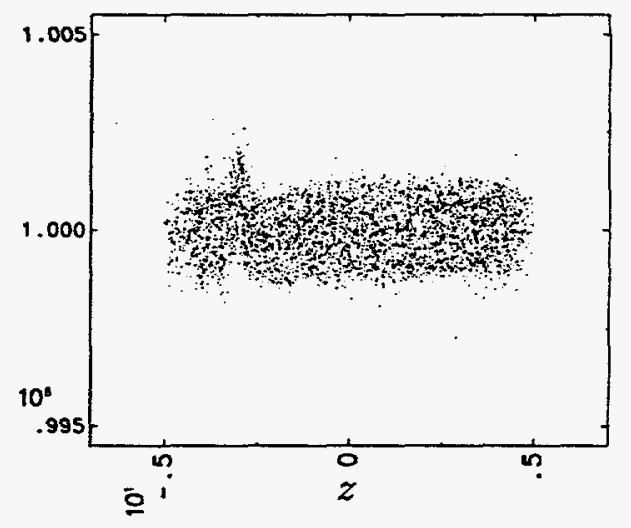

$v_{z}$ vs $z$

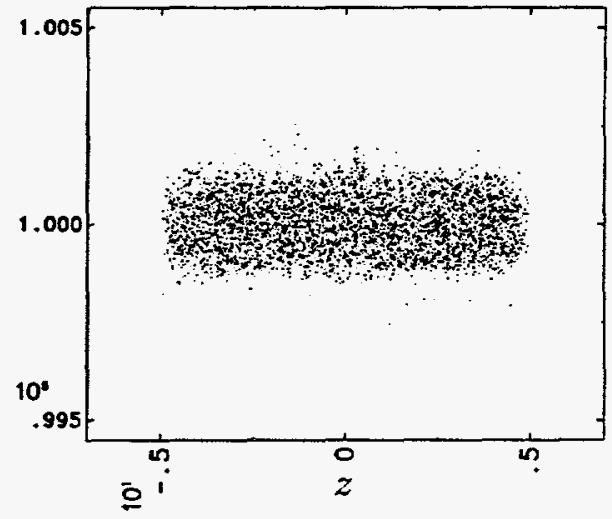

$v_{z}$ vS $z$

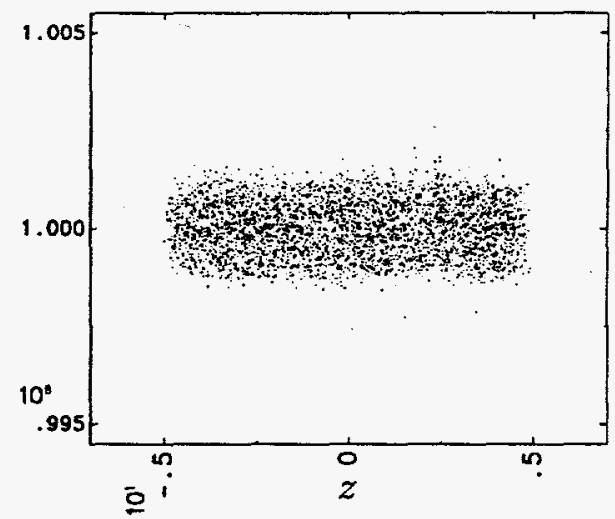

Figure 4.3: The perturbation reflects off the beam end and decays as it travels forward. (a) $28.4 \mu \mathrm{s}$, (b) $35.0 \mu \mathrm{s}$, (c) $39.4 \mu \mathrm{s}$ 
is the case, a complete pass results in overall growth.

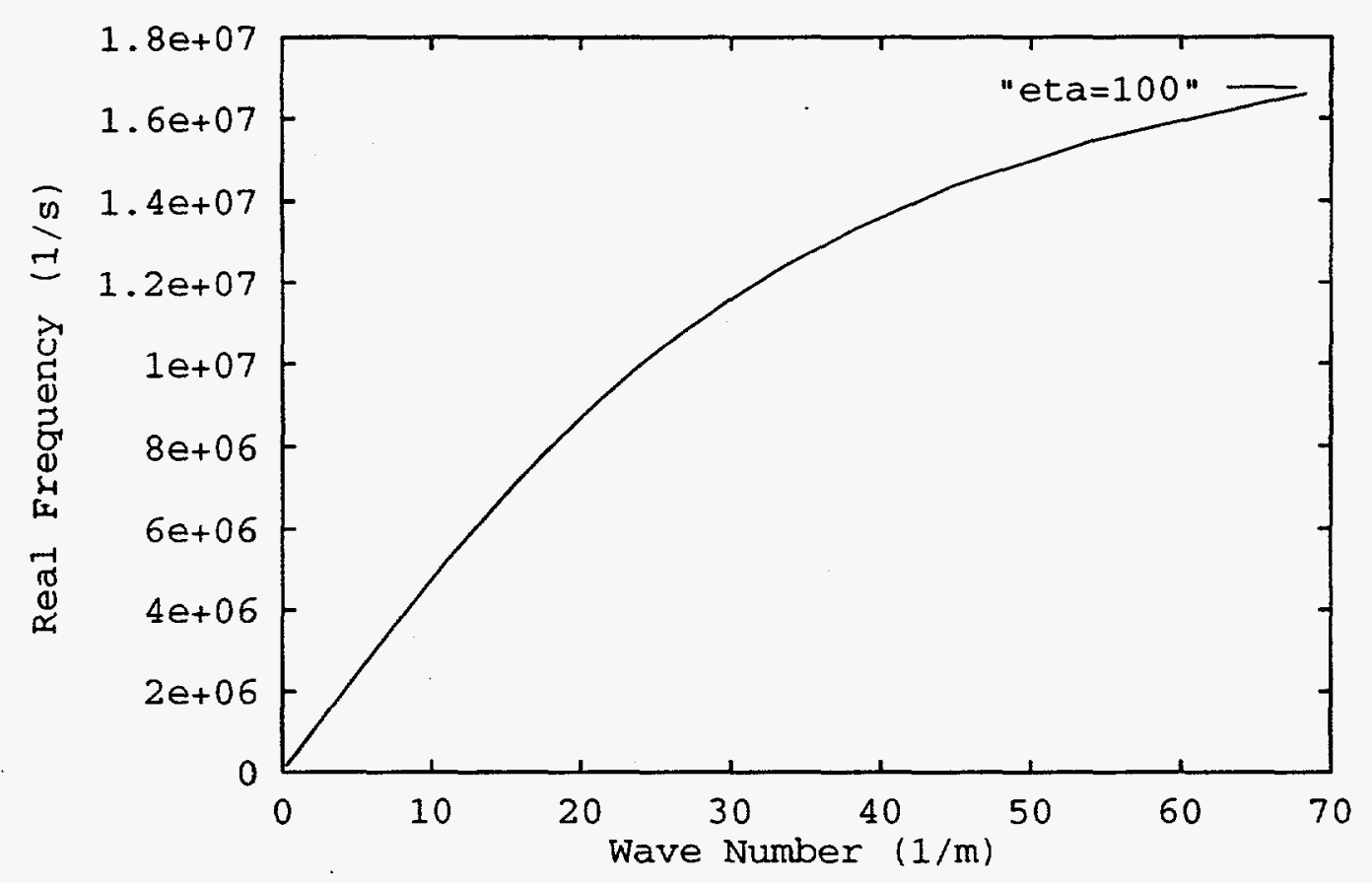

Figure 4.4: Real part of the frequency as a function of wavenumber

While these waves are dispersionless at long wavelengths, there is some dispersion at short wavelengths. The real part of the frequency versus wavenumber for a cold beam is shown in figure (4.4). The plot shows that for small wavenumbers (i.e. long wavelengths since $k=2 \pi / \lambda)$, the real frequency is proportional to the wavenumber and is then dispersionless. For larger wavenumbers (short wavelength), however, the frequency begins to roll over so that $d \omega / d k$ is not independent of $k$. From this, we expect that short wavelengths will have slower group velocities and thus will lag behind the longer wavelengths.

This dispersion is seen clearly as the perturbation travels back towards the beam head (figure (4.3)). Since our initial perturbation was roughly gaussian and thus contains many wavelengths, we expect dispersion to affect the perturbation after enough time has passed for the difference in group velocities to show up. This process has been speeded up, however, by the narrowing of the perturbation during reflection. 
When the perturbation narrows, more of the wavelengths present are in the wavelength regime where dispersion is expected.

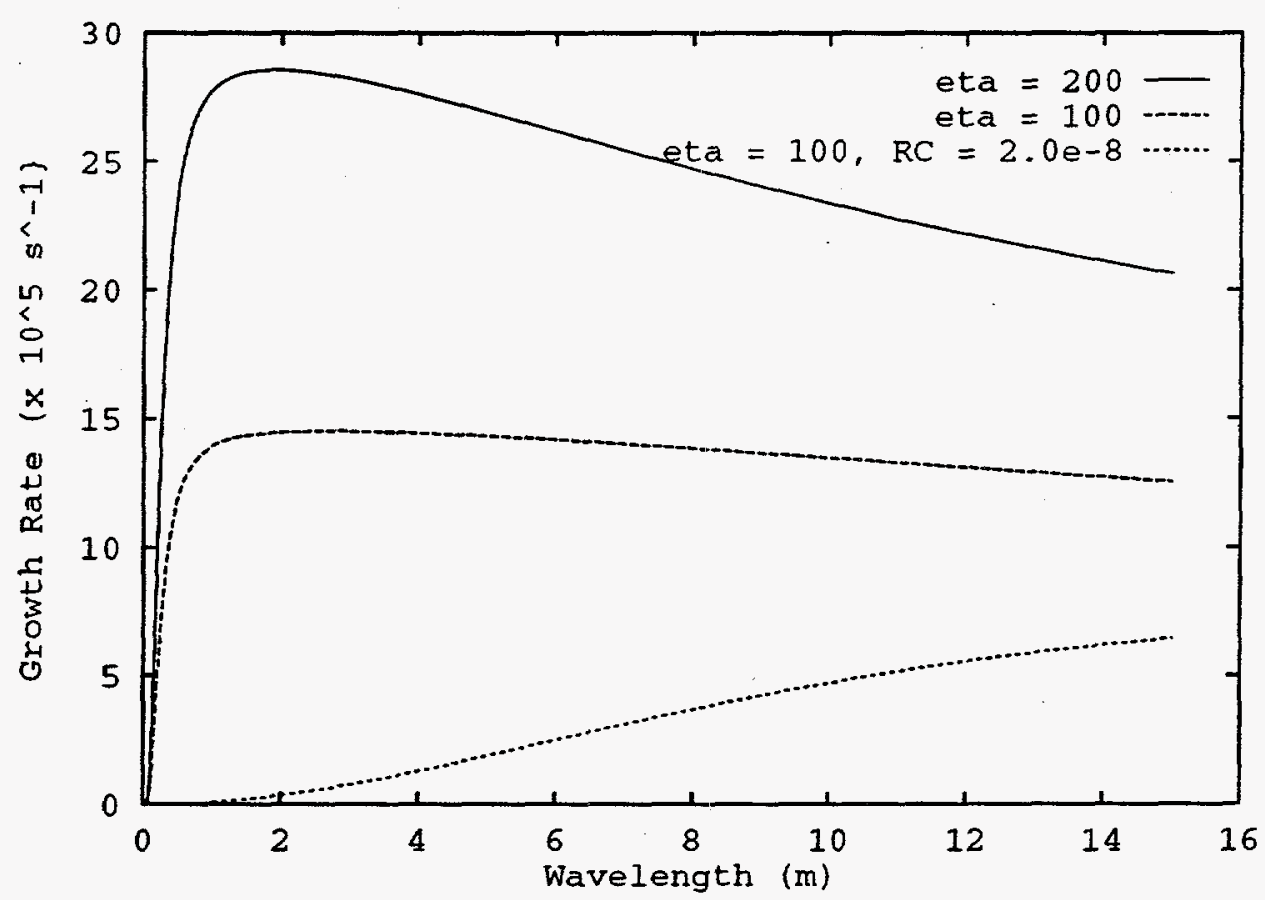

Figure 4.5: Growth rate of the instability vs wavelength for 3 values of the wall impedance

\subsection{Simulations with Resistive and Capacitive Com- ponents}

Cold beam theory predicts that a capacitive component of the module impedance will reduce the growth rate[19] as well as increase the wavelength of the most unstable mode. Figure (4.5) shows the growth rate as a function of wavelength from cold beam fluid theory for the three values of wall impedance used in these simulations.

Adding capacitance also changes the external electric field needed to keep the beam from losing its energy to the wall to

$$
\eta \mathcal{C} \frac{d E_{z, \text { external }}(r, z)}{d t}+E_{z, \text { external }}(r, z)=\eta I_{0}(z)
$$


Again this external field was slightly in error and a perturbation was launched on the beam. In this case, the perturbation launched was very long wavelength (wavelength $\sim$ beam length). The amplitude of the perturbation launched was about 5 times as large as in the case without capacitance, however, as predicted by the cold beam theory, very little growth is observed.

Figure (4.6) shows the electrostatic potential on axis $v s z$ and the $z-v_{z}$ phase space for a case with $100 \Omega / \mathrm{m}$ resistance and an " $R C$ " time of $2.0 \times 10^{-8}$ seconds.

\subsubsection{Summary}

These simulations show the effects of the longitudinal instability of a single perturbation launched from the beam head. In a case with a large resistance, perturbations can grow very large and result in particles being lost. For a more moderate, more realistic resistance, a perturbation launched from the beam head grows as it travels the length of the beam, slows down at it approaches the end, and reflects. Nonlinear effects in the beam end can cause a narrowing of the perturbation during reflection. We see the expected decay of the forward traveling wave, along with dispersion caused by the narrowing in the end.

Both theory and simulations point out the importance of the module capacitance on the growth rate of the longitudinal instability. Including the effect of capacitance greatly decreases the severity of the instability on the beam quality. 
Electrostatic Potential on Axis vs $z$

a)

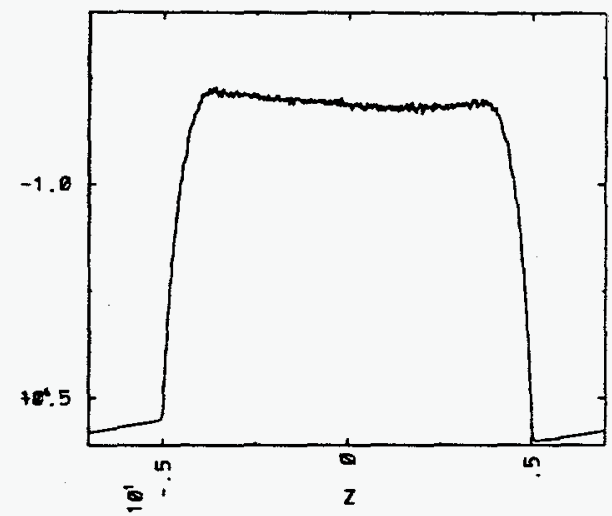

Electrostatic Potential on Axis vs $z$

b)

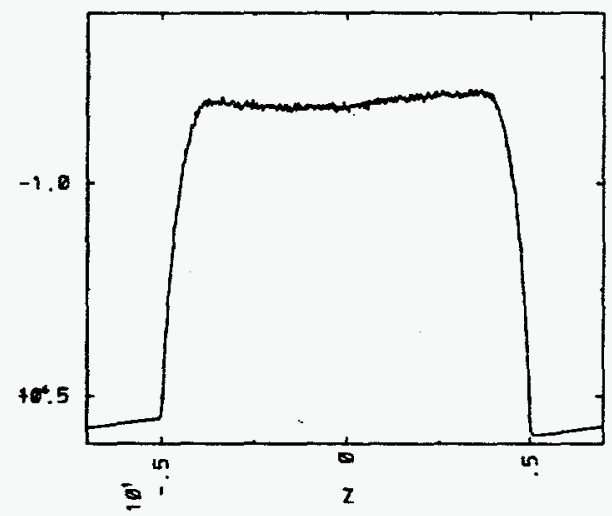

Electrostatic Potential on Axis vs $z$

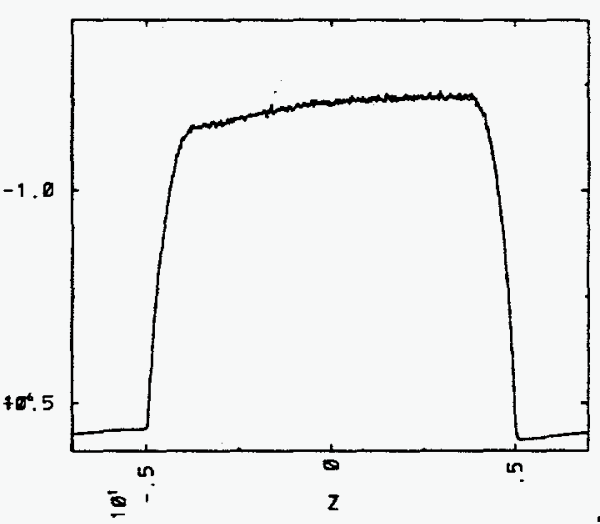

$v_{z}$ VS $z$

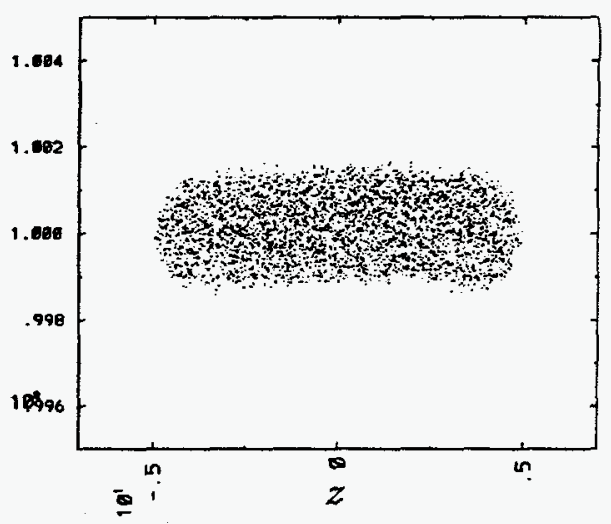

$v_{z}$ vs $z$

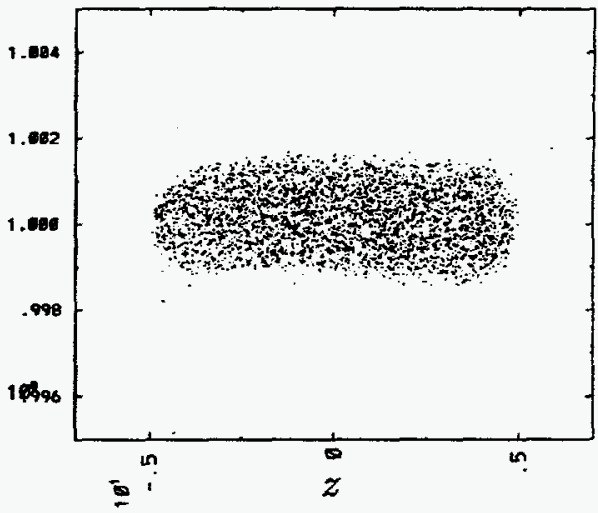

$v_{z}$ vs $z$

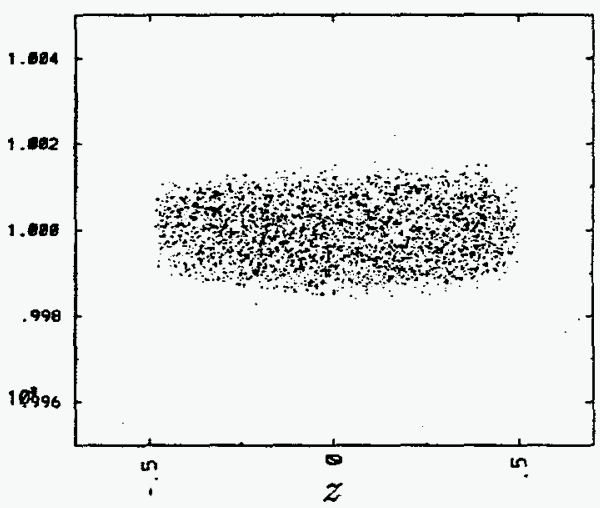

Figure 4.6: When capacitance is added to the system, a larger perturbation is launched, but little growth occurs (a) $6.6 \mu \mathrm{s}$, (b) $10.9 \mu \mathrm{s}$, (c) $17.5 \mu \mathrm{s}$ 


\section{Chapter 5}

\section{Effects of Temperature on Growth Rate}

In the WARPrz simulations discussed in chapter 4, we noticed that the growth rates measured were lower than the growth rates predicted by the cold beam dispersion relation by $10-20 \%$. The dispersion relation derived in chapter 3 assumes the following:

- The beam is periodic and infinite in length.

- The perturbation is sinusoidal.

- The perturbation is small so the calculation is in the linear regime (ie the line charge density perturbation is small compared with the line charge density).

- The beam has no thermal spread in either the direction parallel or perpendicular to the direction of travel.

The test cases described in section 3.7.2 satisfy each of these criteria and the growth rates agree with the theory to within $1.5 \%$.

The simulations shown in chapter 4 are not periodic, infinite beams and the perturbations launched in those simulations are not sinusoidal. However, simulations with periodic, infinite beams with sinusoidal perturbations that include thermal spread in both the parallel and perpendicular directions also show growth rates lower than the 
cold beam theory predicts. This stabilization is due to the finite temperatures in the beams in these simulations. Thermal velocities of the particles move them into and out regions of the backward wave and thus smooth out some of the effects of the instability.

\subsection{Including Longitudinal Temperature}

Our first attempt to quantify the stabilization due to finite temperature effects was to include the longitudinal temperature in a long wavelength description of the beam. This derivation follows that of Wang and Reiser[26]

We begin with the linearized Vlasov equation,

$$
\left(\frac{\partial}{\partial t}+v \frac{\partial}{\partial z}\right) f_{1}(z, v, t)=-\frac{q}{m} E_{1} \frac{\partial f_{0}}{\partial v}
$$

In the long wavelength limit, the electric field is given by

$$
E_{1}=-g \frac{\partial \lambda_{1}}{\partial z}-\eta I_{1}
$$

We assume that all perturbed quantities vary as $\exp (i k z-i \omega t)$

$$
f_{1}=\frac{q}{m}\left(-k g \lambda_{1}+i \eta I_{1}\right) \frac{\partial f_{0} / \partial v}{(\omega-k v)}
$$

The continuity equation relates the perturbed current, $I_{1}$, to the perturbed line charge density, $\lambda_{1}$,

$$
I_{1}=\frac{\omega}{k} \lambda_{1}
$$

We also use

$$
\lambda_{1}=\lambda_{0} \int f_{1} d v
$$

to get the dispersion relation,

$$
1=\frac{q \lambda_{0}}{m}\left(-k g+i \frac{\eta \omega}{k}\right) \int_{-\infty}^{\infty} \frac{\partial f_{0} / \partial v}{\omega-k v} d v
$$


This dispersion relation can be rewritten in a more useful form by integrating by parts and assuming that the zero order distribution function goes to zero as the velocity becomes very large.

$$
1=\frac{q \lambda_{0}}{m}\left(k^{2} g-i \eta \omega\right) \int_{-\infty}^{\infty} \frac{f_{0}}{(\omega-k v)^{2}} d v
$$

We can check this dispersion relation in the cold beam limit by using a delta function distribution function.

$$
f_{0}=\delta\left(v-v_{b}\right)
$$

This gives the dispersion relation

$$
1=\frac{k^{2} v_{p}^{2}}{\left(\omega-k v_{b}\right)^{2}}-i \eta \frac{v_{p}^{2}}{g} \frac{\omega}{\left(\omega-k v_{b}\right)^{2}}
$$

where we've again introduced $v_{p}^{2}=q \lambda_{0} g / m$. This agrees with the dispersion relation that we got in the cold beam, 1-d theory in the lab frame.

In the case of a Gaussian distribution,

$$
f_{0}=\frac{\exp \left[-\left(v-v_{b}\right)^{2} / v_{\mathrm{th}}^{2}\right]}{\sqrt{\pi} v_{\mathrm{th}}}
$$

we use the derivative of the plasma dispersion function,

$$
Z^{\prime}(\xi)=\frac{1}{\sqrt{\pi}} \int_{-\infty}^{\infty} \frac{e^{-x^{2}}}{(x-\xi)^{2}} d x
$$

and the relationship between $Z$ and $Z^{\prime}$

$$
Z^{\prime}(\xi)=-2(1+\xi Z(\xi))
$$

Using these relationships gives the dispersion relation,

$$
1=\frac{2 v_{p}^{2}}{\left(k v_{\mathrm{th}}\right)^{2}}\left[-k^{2}+i \frac{\eta k}{g}\left(\xi v_{\mathrm{th}}+v_{b}\right)\right](1+\xi Z(\xi))
$$

where $\xi=\left(\omega-k v_{b}\right) /\left(k v_{\text {th }}\right)$ is the frequency in the beam frame divided by $k v_{\text {thl }}$.

We can again compare with the cold beam theory by using the large argument expansion of the plasma dispersion function.

$$
Z(\xi) \simeq-\xi^{-1}(1+1 /(2 \xi))
$$


in the case where $\xi_{\text {imaginary }}>1 /\left|\xi_{\text {real }}\right|$. Using this expansion, gives

$$
1=\frac{2 v_{p}^{2}}{\left(k v_{\text {th }}\right)^{2}}\left[-k^{2}+i \frac{\eta k}{g}\left(\xi v_{\text {th }}+v_{b}\right)\right]\left(\frac{-1}{2 \xi^{2}}\right)
$$

Using the definition of $\xi$ once again gives the cold beam dispersion relation in the lab frame,

$$
1=\frac{v_{p}^{2}}{\left(\omega-k v_{b}\right)^{2}}\left(k^{2}-i \frac{\eta}{g} \omega\right)
$$

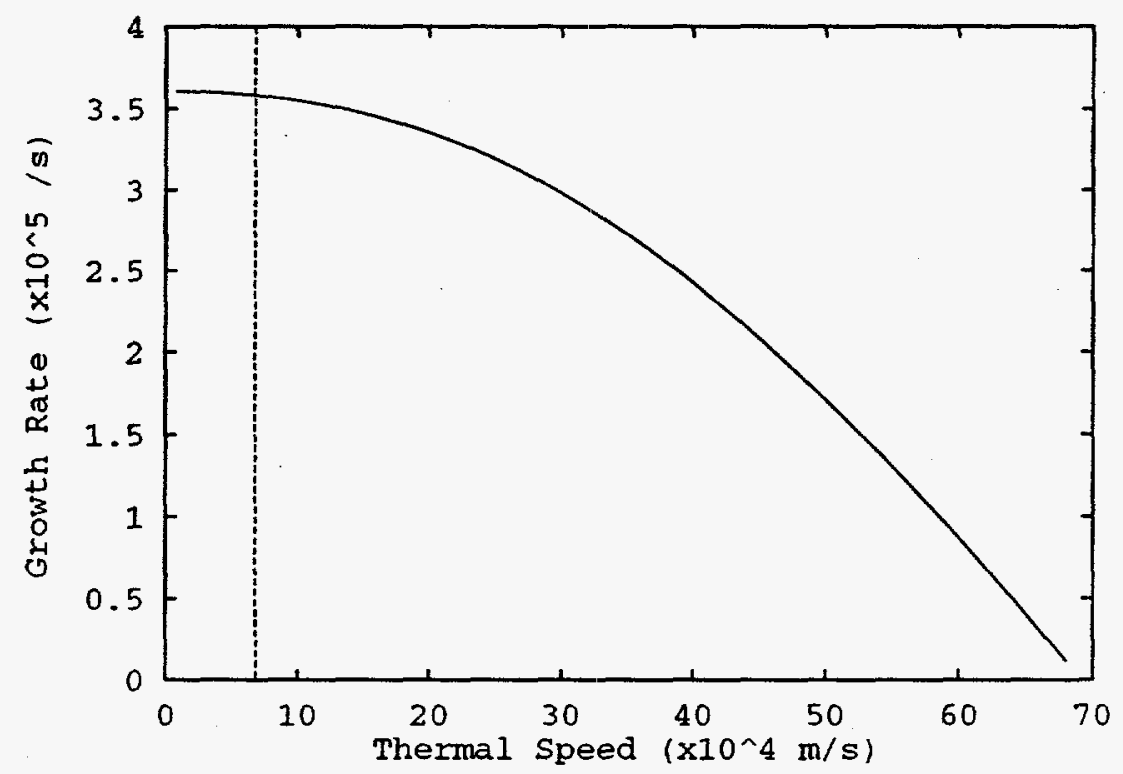

Figure 5.1: Growth rate as a function of longitudinal thermal speed. The vertical line represents the largest thermal speed that can be focused to the necessary spot size.

We can evaluate the plasma dispersion function numerically and graph equation (5.13) to see the effect of longitudinal temperature on the dispersion relation in the long wavelength limit. Figure (5.1) shows the growth rate of the instability as a function of the longitudinal thermal speed for the parameters in table 5.1 and wavenumber times pipe radius $k b=.06$. The vertical line in figure (5.1) indicates a thermal speed of $6.8 \times 10^{4} \mathrm{~m} / \mathrm{s}$, which corresponds to the largest longitudinal temperature that can 


\begin{tabular}{|l|r|}
\hline lon mass & $200 \mathrm{amu}$ \\
\hline Ion charge state & +1 \\
\hline Beam velocity & $1 / 3 \mathrm{c}$ \\
\hline Beam current & .4 \\
\hline Beam radius/Pipe radius & 3000 Amps \\
\hline Wall resistance & $300 \Omega / \mathrm{m}$ \\
\hline Perturbation wavelength & 6 meters \\
\hline
\end{tabular}

Table 5.1: Parameters used in figure (5.1)

be focused on the target $(10 \mathrm{keV})$. This shows that for acceptable thermal speeds, there is no appreciable change in the growth rate of the instability.

If we run WARPrz with the same parameters used to calculate figure(5.1) and a thermal speed of $6.8 \times 10^{4} \mathrm{~m} / \mathrm{s}$ in both the parallel and perpendicular directions, we measure a growth rate of $3 \times 10^{5} \mathrm{~s}^{-1}$. This growth rate is smaller than the cold beam theory growth rate by only about $15 \%$. Figure (5.1) shows that to get this reduction in the growth rate from longitudinal temperature alone, we would need a thermal speed almost 4.5 times the size of the one we used in the WARPrz run.

From this theory, we conclude that a temperature spread parallel to the direction of beam travel should have no appreciable effect on the growth rate of the longitudinal instability. However, we do see an appreciable decrease in the growth rate of the instability when the beam has finite temperature in the WARPrz runs. This leads us to believe this decrease in growth rate is due to temperature in the direction perpendicular to the direction of travel.

\subsection{A Coupling Mechanism for Perpendicular Tem- perature and Wave Damping}

The complete theory including finite perpendicular temperature is a difficult problem and so we have tried to come up with a simpler model which may describe the essential physics of the damping observed.

The simple model only includes the radial direction. If we sit at one $z$ value in 
the beam frame, then as a sinusoidal wave passes by, we see the beam radius vary sinusoidally in time. A particle moving in the radial direction will move towards the edge of the beam (at the beam radius) and eventually reflect off that beam edge. In reality, the beam does not have a sharp radial edge to it, but a sheath. For a space charge dominated beam, the width of this sheath is approximately the Debye length so if the beam radius is large compared with the transverse Debye length, then we can consider it a "hard" edge.

Our simple model consists of particles and a wall which oscillates in and out at the wave frequency. If a particle reflects off the wall when the wall is moving inward, it gains energy from the wall. Likewise, a particle which reflects off the wall when the wall is moving outward loses energy to the wall. If a particle has the correct velocity so that it was "in resonance," it could reflect off the wall when the wall is moving in, travel across the beam, and again reflect off the wall when the wall is moving in. This could happen many times until the particle gains enough energy to be out of resonance.

For a particle to be in resonance, the time it takes for the particle to cross the beam diameter would have to equal a multiple of the period of the wave. The time it takes for the particle to cross the beam radius is

$$
\tau_{\text {cross }}=\frac{2 a}{v_{r}}
$$

where $a$ is the beam radius and $v_{r}$ is the particle velocity. Setting this equal to the period of the wave,

$$
\tau_{\text {cross }}=\frac{2 a}{v_{r}}=\frac{2 \pi}{\omega}=T_{\text {wave }}
$$

Solving for the resonant velocity,

$$
v_{r}=\frac{a \omega}{\pi}=\frac{k a v_{p}}{\pi}
$$

where the wave frequency has been rewritten in terms of the wave phase velocity, $v_{p}$. In fact, while particles traveling at the velocity described in equation (5.19), will gain the largest amount of energy from the wave, particles at $1 / 2,1 / 3,1 / 4$, etc of $v_{r}$ can 


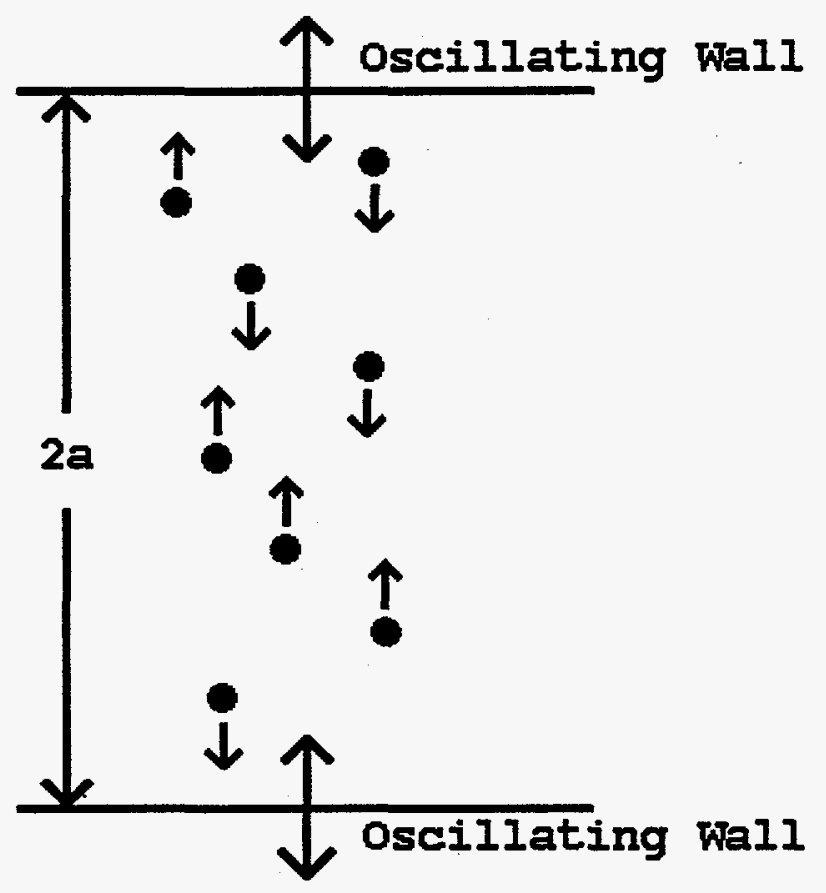

Figure 5.2: The simple model approximates the surface wave as an oscillating wall which particles reflect off.

also gain energy from the wave. Particles with velocity $(1 / 2) v_{r}$ will bounce off the wall every other wave period and particles with velocity $(1 / 3) v_{\tau}$ will hit every third wave oscillation. So in general, the resonance condition is

$$
v_{r, n}=\frac{k a v_{p}}{n \pi}
$$

where $n=1,2,3, \ldots$

A theoretical treatment of this model was done by Langdon[18]. This theory showed the resonances described above and predicted an increase in particle energy from the oscillating wall. Connecting this theory to the results of the simple code described in the next section and to the results of WARPrz simulations will be done in the future. 


\subsection{A Code Using the Simple Model}

We wrote a simple code to look for an increase in particle energy when the particle reflects off an oscillating hard wall. This model is one dimensional and also neglects collective interactions. Instead, we simply have particles which move with constant velocity across the beam and only undergo changes in velocity when they hit the wall.

We can work out the change in velocity that the particle undergoes when it hits the wall by considering two masses, $m$ and $M$, with initial velocities, $v$ and $V$. After the perfectly elastic collision, the particles have velocities $v^{\prime}$ and $V^{\prime}$. There exists a center of momentum frame such that

$$
m v_{c}+M V_{c}=0
$$

where the subscript denotes velocity in the center of momentum frame. We can transform the center of momentum frame velocities into the lab frame using a Galilean velocity shift,

$$
\begin{gathered}
v=v_{c}+v_{f} \\
V=V_{c}+v_{f}
\end{gathered}
$$

where $v_{f}$ is the velocity of the frame. We can get the velocity of the center of momentum frame in terms of the particle masses and particle lab frame initial velocities by substituting equations (5.22) and (5.23) into equation (5.21) and solving for $v_{f}$,

$$
v_{f}=\frac{V+\frac{m}{M} v}{1+\frac{m}{M}}
$$

We are interested in getting the velocity of the small mass after the collision. In the center of mass frame,

$$
v_{c}^{\prime}=-v_{c}
$$

So

$$
v^{\prime}=v_{c}^{\prime}+v_{f}=-v_{c}+v_{f}
$$

Using equation (5.22),

$$
v^{\prime}=-v+2 v_{f}
$$


In the limit that the large mass $(M)$ becomes large compared to the particle mass $(m)$, the velocity of the center of mass frame (equation (5.24)) becomes just

$$
v_{f}=V
$$

So the particle reflecting off the wall has a velocity after the collision of

$$
v^{\prime}=-v+2 V
$$

where $V$ is the velocity of the wall.

Using this result, we have written a simple code which tracks particles bouncing off a moving wall. The particles are loaded uniformly across the beam radius and given a Gaussian distribution of velocities. The particles move with constant speed until they hit the sinusoidally oscillating wall. When they hit the wall, their velocity is changed by the amount dictated by equation (5.29).

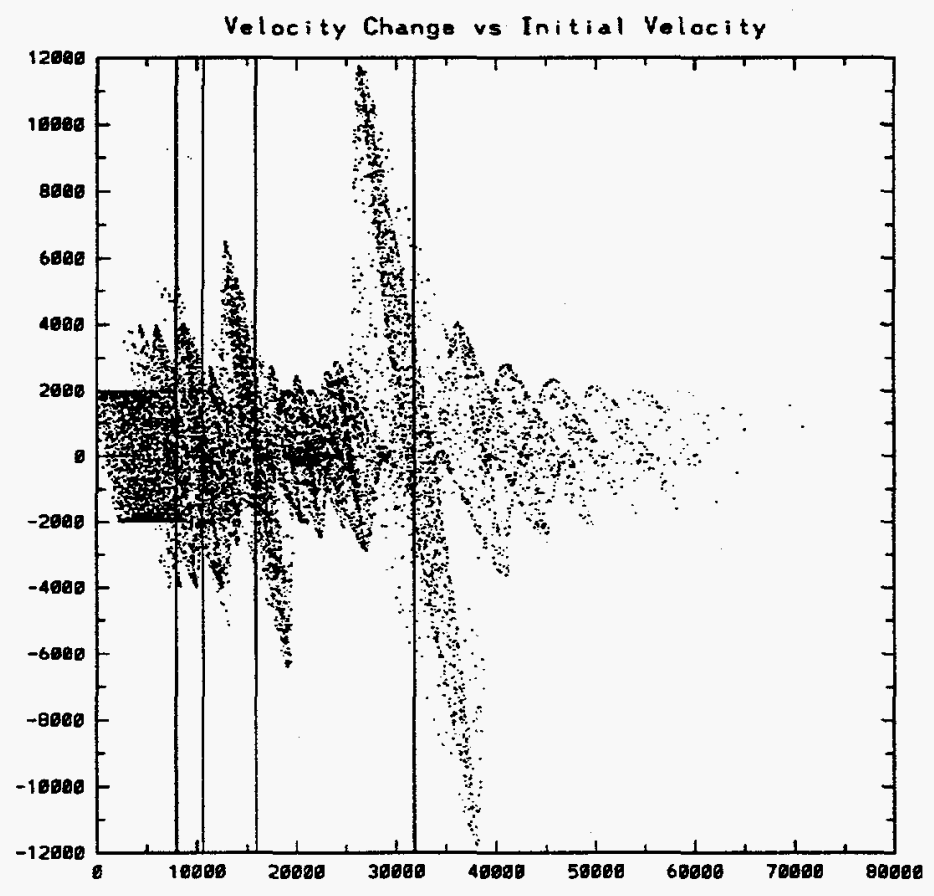

Figure 5.3: Resonances are seen for particles which reflect off the oscillating wall every $1,2,3$, and 4 oscillations. Vertical lines indicate resonant velocities. 
If we run this simple "wall and ball" code, we expect particles with initial velocities near the resonant velocities (given in equation (5.20)) to undergo large velocity changes. This can be seen clearly in figure (5.3) where we plot the change in the speed each particle, $\left|v_{\text {fual }}\right|-\left|v_{\text {initial }}\right|$ against the particle's initial speed, $\left|v_{\text {initial }}\right|$. In this run, the initial thermal spread was $2.0 \times 10^{4} \mathrm{~m} / \mathrm{s}$. The beam radius was $.025 \mathrm{~m}$ and the wall variation was $1 \%$ of the beam radius. The wall oscillated at a frequency of $4.0 \times 10^{6} \mathrm{~s}^{-1}$. Putting these parameters into equation (5.20)), gives resonances at $\left(3.18 \times 10^{4}\right) / n \mathrm{~m} / \mathrm{s}$. In the figure, we can clearly see the large change in particle speed for $n=1,2,3,4$.

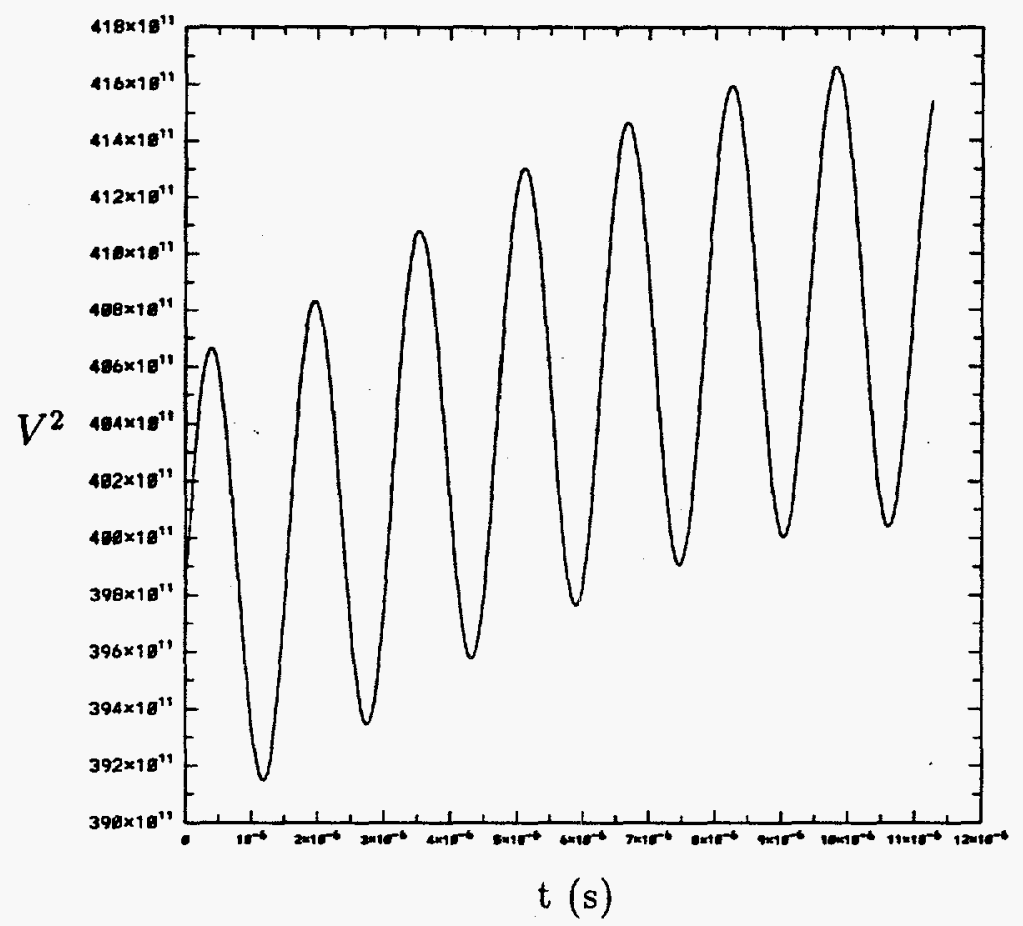

Figure 5.4: Sum of the square of the particle velocities (proportional to the total energy) increases with time as the particles gain energy from the oscillating wall. The increase in energy levels off as the distribution function is flattened in the region surrounding the resonance. The oscillations are at the wall oscillation frequency.

Figure (5.3) shows that particles with velocities slightly lower than the resonant velocity gain energy, while particles with velocity slightly higher than the resonant velocity tend to lose energy. This means that total particle energy will increase only 
if the slope of the distribution function is negative at the resonances. If the slope is negative, there the number of particles that gain energy will be larger than the number of particles which will lose energy. Since we are using a Gaussian distribution for particle velocities, the slope is, in fact, negative and we see an increase in the total energy of the particles (figure (5.4)).

Figure (5.5) shows the distribution function of the particles as a function of velocity at the beginning and the end of the run. We see that the distribution has flattened out in the region surrounding the resonance at $\left(3.18 \times 10^{4}\right) \mathrm{m} / \mathrm{s}$. This resonance has the largest effect on the particle energy because it represents particles which reflect off the wall every time the wall oscillates. From the distribution function, we expect the damping mechanism to saturate soon after this plot was made because the distibution function has become flat in this region. This is also indicated in figure (5.4) as the energy increase levels off near the end of the run. Longer runs show the particles stop gaining energy altogether a short time later.

This saturation due to flattening of the distribution function occurs earlier in time when the wall oscillations are larger. Large oscillations lead to large changes in particle velocity at each reflection. These large changes in particle velocity cause the distribution function change more rapidly and thus the saturation occurs more rapidly.

With this simple model, we have shown that particles moving perpendicular to the direction of wave motion can gain energy from the wave if the time it takes for the particle to cross the beam diameter is an integer number of wave periods. This increase in the particle energy must come at the expense of the wave energy.

\subsection{Growth Rates with Finite Temperature from WARPrz}

We begin with one of the simulations which lead us to look for finite temperature effects on the wave growth rate. This is the simulation discussed in the first section 
(a)

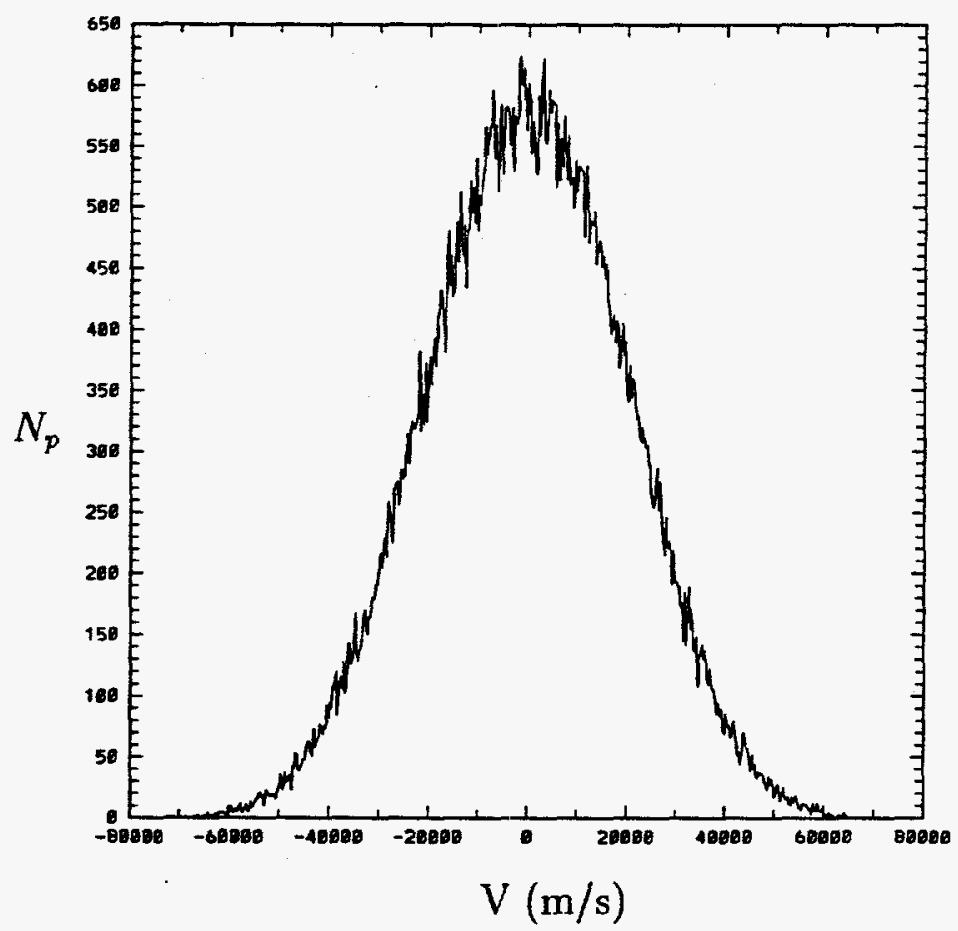

(b)

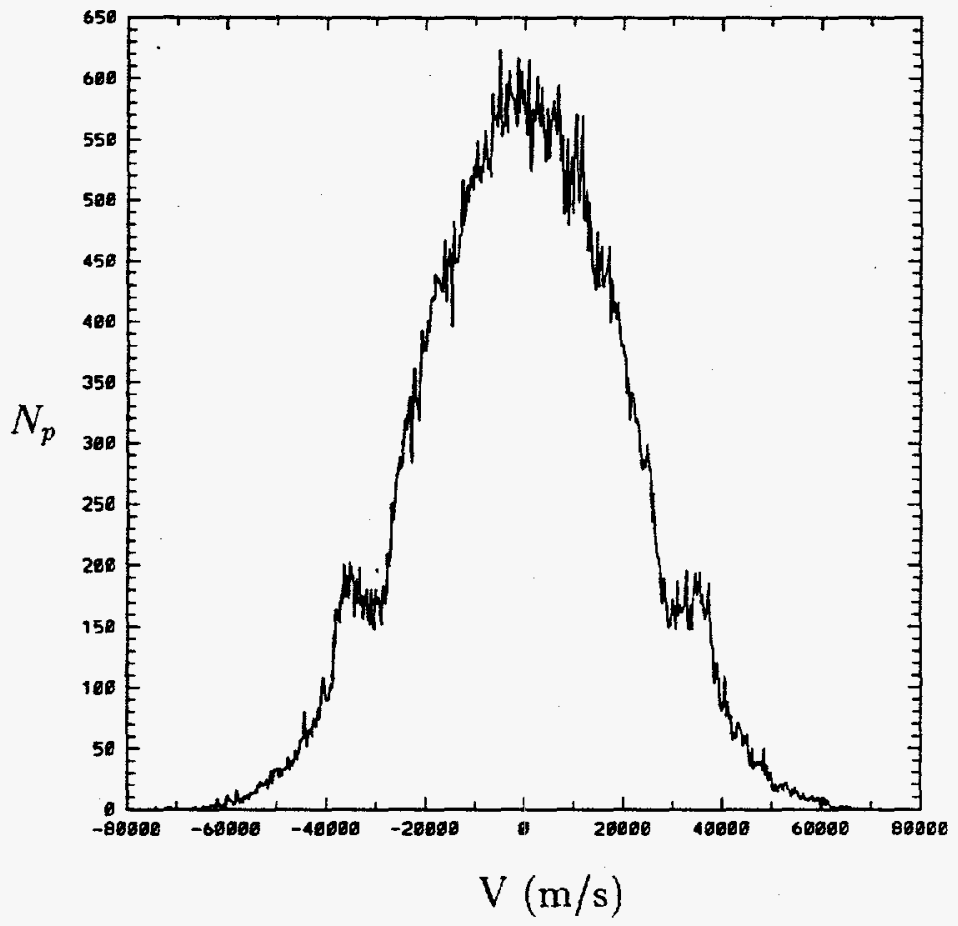

Figure 5.5: The distribution of particles as a function of velocity at the beginning of the run (a) and the end of the run (b) shows the distribution flattens out at the $n=1$ resonance 
of this chapter and the parameters are summarized in table (5.1). In this case, the cold beam theory predicted a growth rate of $3.6 \times 10^{5} \mathrm{~s}^{-1}$ but the measured growth rate was $3.0 \times 10^{5} \mathrm{~s}^{-1}$. The growth rate was measured by the following procedure:

1. A sinusoidal velocity perturbation is excited in a periodic beam.

2. The corresponding perturbation in line charge density "grows" from the velocity perturbation.

3. At specified intervals in time, we take the FFT of the line charge density with respect to space. We then select the coefficents of the mode that was excited and save these coefficients until the end of the run.

When we FFT, we are expressing

$$
\lambda(z, t)=\Sigma_{i=0}^{N-1} \hat{\lambda}\left(k_{i}, t\right) e^{i k_{i} z}
$$

where $\lambda$ and $\hat{\lambda}$ are complex quantities and $N$ is the number of discrete modes allowed. If we have a backward traveling wave moving with velocity $\omega / k$ and growing exponentially with growth rate $\gamma$ then $\lambda$ can be written as

$$
\lambda(z, t)=\cos (k z+\omega t) e^{\gamma t}
$$

So that

$$
\begin{gathered}
\hat{\lambda}_{\text {real }}=\cos (\omega t) e^{\gamma t} \\
\hat{\lambda}_{\text {imag }}=-\sin (\omega t) e^{\gamma t}
\end{gathered}
$$

If we take the sum of the squares of the real and imaginary parts of the FFT,

$$
\hat{\lambda}_{\text {real }}^{2}+\hat{\lambda}_{\text {imag }}^{2}=e^{2 \gamma t}
$$

4. Using equation (5.34), the coefficients from the FFT are squared and then the natural $\log$ is taken. This is plotted against time and the slope of the resulting straight line is twice the growth rate. 
5. Equation (5.34) assumes only a backward wave. By starting a perturbation in velocity only, we have excited both a backward and a forward wave. Since the forward wave damps, we simply wait an e-folding or two for the forward wave to become very small compared to the backward wave and then measure the slope of the straight line to get the growth rate.

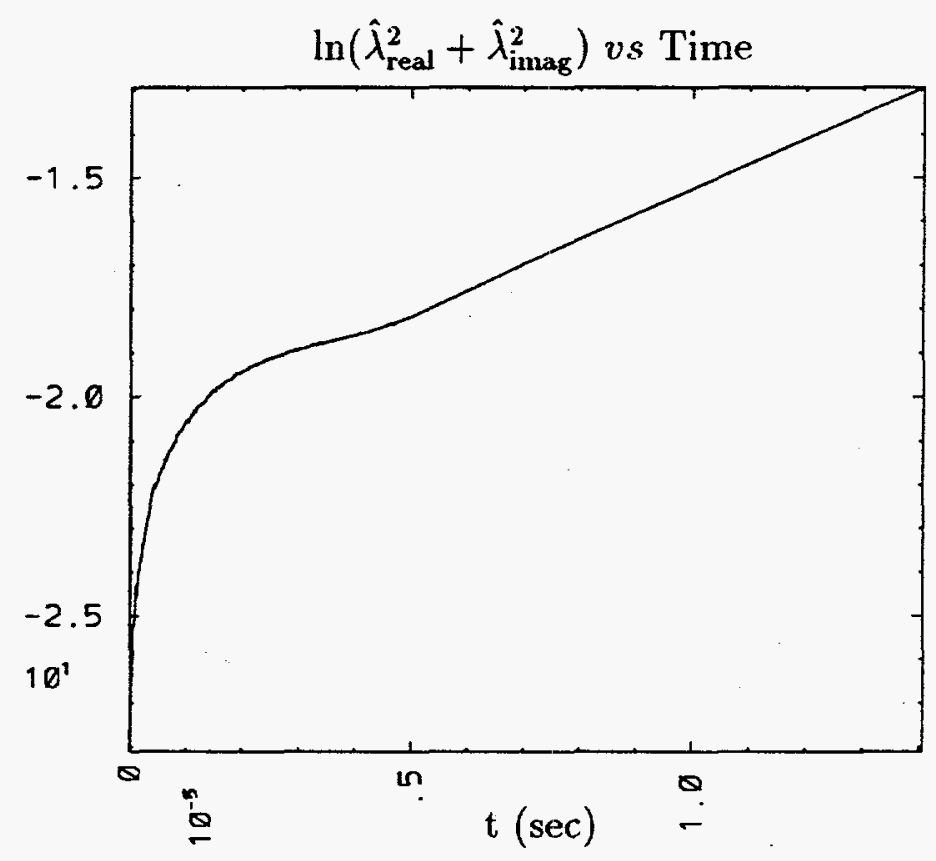

Figure 5.6: The slope of the straight line is equal to twice the growth rate

Figure (5.6) shows the natural $\log$ of the square of the FFT coefficients as a function of time for the parameters in table (5.1). In about the first microsecond, the amplitude of the line charge density grows very rapidly. This happens because the perturbation was initially started in the velocity only and the corresponding perturbation in line charge density grows up from that. In the next 4 microseconds, the forward traveling wave is decaying. After about 5 microseconds, we see a straight line emerge. One half of the slope of this straight line is the growth rate for the backward wave. 
If the simple "wall and ball" model is correct, we expect the temperature to stabilize the mode more completely when the wave frequency is higher. This is true because

1. A higher wave frequency means more wall oscillations in a given amount of time and thus, more chances for the particles to bounce off the wall and gain energy from it.

2. A higher wave frequency means a higher resonant velocity for the particles. Since we've seen that the slope of the distribution function determines whether the particles gain energy overall, we expect that the particles will gain the most energy when the resonant velocity is close to the thermal speed.

\begin{tabular}{|c|c|c|c|c|c|c|}
\hline Run & $\lambda$ & $\omega_{\text {real }}$ & $v_{r} / v_{\text {th }}$ & $k b$ & $\gamma$ calculated & $\gamma$ measured \\
\hline $\mathrm{a}$ & 6.000 & $5.1 \times 10^{5}$ & .06 & 0.07 & $3.6 \times 10^{5}$ & $3.0 \times 10^{5}$ \\
\hline $\mathrm{b}$ & 1.500 & $2.0 \times 10^{6}$ & .24 & 0.26 & $4.2 \times 10^{5}$ & $2.2 \times 10^{5}$ \\
\hline $\mathrm{c}$ & 0.750 & $4.1 \times 10^{6}$ & .48 & 0.53 & $4.0 \times 10^{5}$ & $\approx 0.0$ \\
\hline $\mathrm{d}$ & 0.375 & $8.2 \times 10^{6}$ & .96 & 1.06 & $3.0 \times 10^{5}$ & $\approx 0.0$ \\
\hline
\end{tabular}

Table 5.2: Varying the wavelength (and thus, the wave frequency) increases the stabilization of the mode

We can increase the wave frequency most easily by changing the wavelength since $\omega_{\text {real }} \approx k v_{p}$. Table (5.2) shows the results of four runs. The cold beam theory predicts the most unstable wavelength for these parameters at about $\lambda=1.5$ meters. At this wavelength, the warm beam WARPrz simulations show the growth rate is close to half the calculated cold beam value! In the higher frequency cases, the mode is almost completely stabilized!

Figure (5.7) shows the log of the modulus squared of the FFT of the line charge density as a function of time for case (c). Unlike case (a) (shown in figure (5.6)), there is no growth seen. If the growth rate predicted by the cold beam theory were correct, this system would have undergone three e-foldings of growth in the time shown in figure (5.7). The humps in figure (5.7) occur at twice the wave frequency and are the 


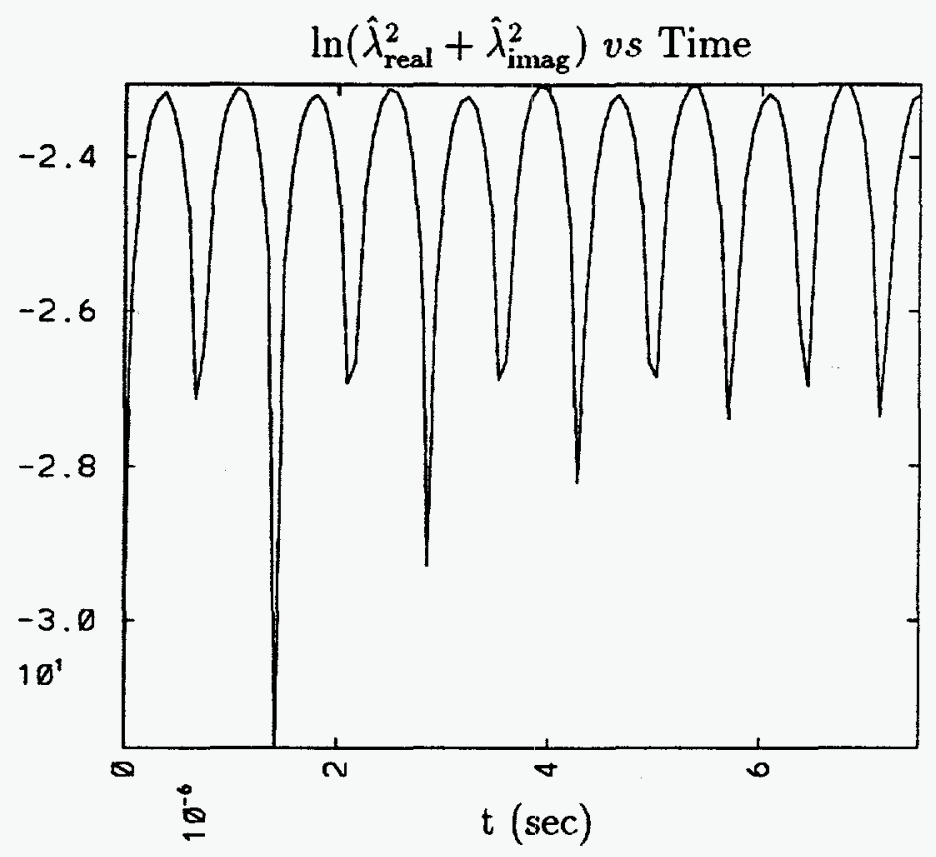

Figure 5.7: No growth is seen in case (c)

result of the backward and forward waves interfering constructively and destructively with each other.

This figure brings up another question-why is the forward wave no longer damping? In these runs, not only is the growth rate of the backward traveling wave reduced, but the decay rate of the forward traveling wave is also reduced. We first thought that somehow energy was being transfered from the backward wave into the forward wave. However, a run which had only a backward wave (no forward wave) also showed almost no growth for the same parameters.

If the "wall and ball" model is correct, then we expect the particles to be gaining energy in the direction perpendicular to the beam motion. If the particles gain large amounts of thermal velocity from the damping of the wave, it could prevent us from being able to focus the beam onto the target at the end of the heavy ion fusion driver which was the reason we were worried about the longitudinal instability in the first place. However, if we plot the perpendicular particle energy as a function of time for 


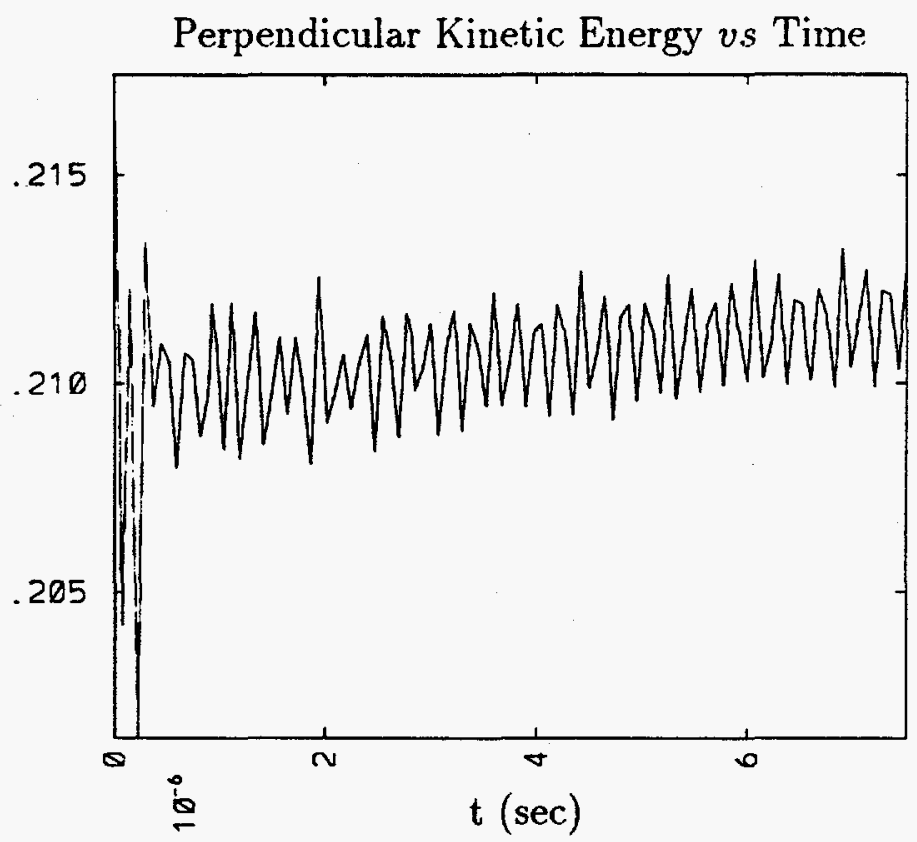

Figure 5.8: Although the wave is not growing, the increase in perpendicular particle kinetic energy (Joules) is less than $1 \%$ over more than 7 microseconds.

case (c) (figure (5.8)), we see that the energy increases by less than $1 \%$.

If the "wall and ball" model is correct, we also expect without resistance present, waves should damp. We tried to maximize this effect by making the perturbation as large as we could without having nonlinear effects. We started an $8 \%$ perturbation in the particle velocity and let that perturbation split into a $4 \%$ forward wave and a $4 \%$ backward wave. Figure (5.9) shows the field energy as a function of time for this run. Since there are two waves present, we again see the constructive and destructive interferring of the waves which gives the field energy oscillations at twice the wave frequency. We see the field energy decrease by about .01 Joules. Figure (5.10) shows the perpendicular kinetic energy. A change in perpendicular energy of .01 Joules would be visible over the noise in this plot, however, we do not see such an increase.

It appears that our simple "wall and ball" model is too simplistic to explain the results of the WARPrz simulations. In the last simulation in which there was no 


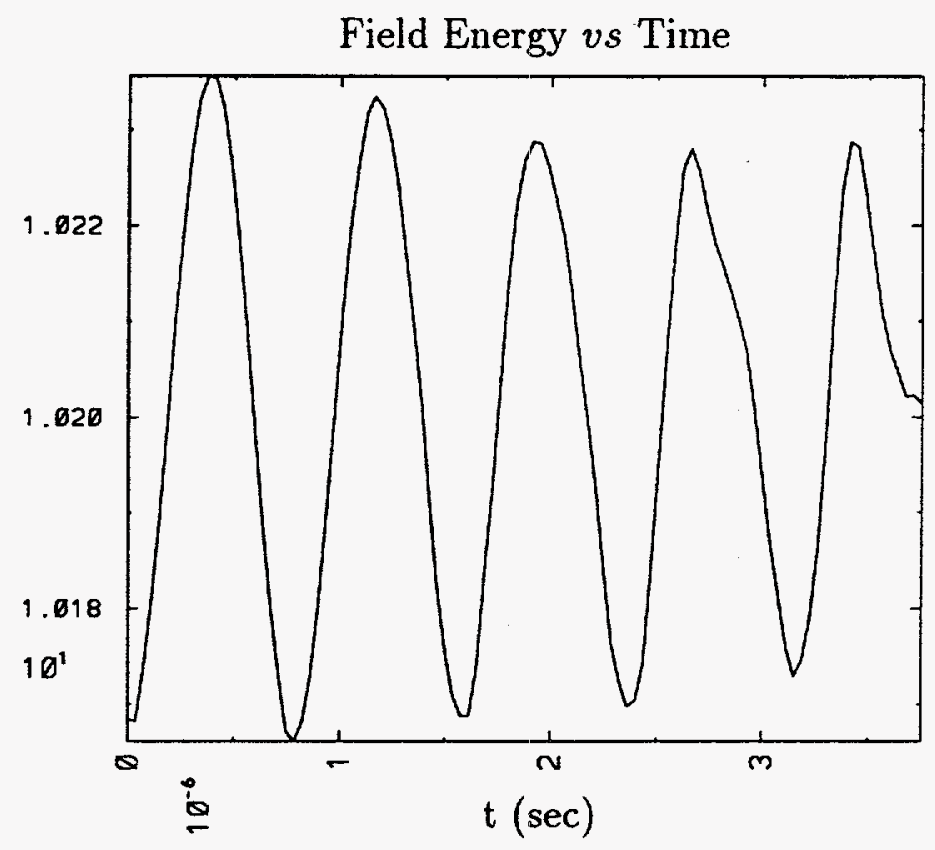

Figure 5.9: When no resistive wall is present, we expect the wave to decay. Here we see the field energy decrease when a $4 \%$ forward and a $4 \%$ backward wave are excited.

resistive wall present, we see a decrease in wave energy of only .01 Joules. We do not see an increase in perpendicular kinetic energy corresponding to this decrease in field energy. Could energy be transferred from the perpendicular direction to the parallel direction? If so, this would not be accounted for in the "wall and ball" model. Can we observe this?

Currently, the answer is no. When the wave is excited, half the energy in the wave goes into field energy and half goes into particle energy and the wave energy oscillates between these two. This is also true in a Langmuir wave and the physics is easy to see in that case. If we think of a Langmuir wave as an electron oscillating about an ion, then when the electron is farthest away from the ion and turning around, the electric field (and hence the field energy) is largest but the kinetic energy is the smallest. When the electron is close to the ion, it is traveling at its highest speed so the kinetic energy is large, but the field energy is small because the separation 


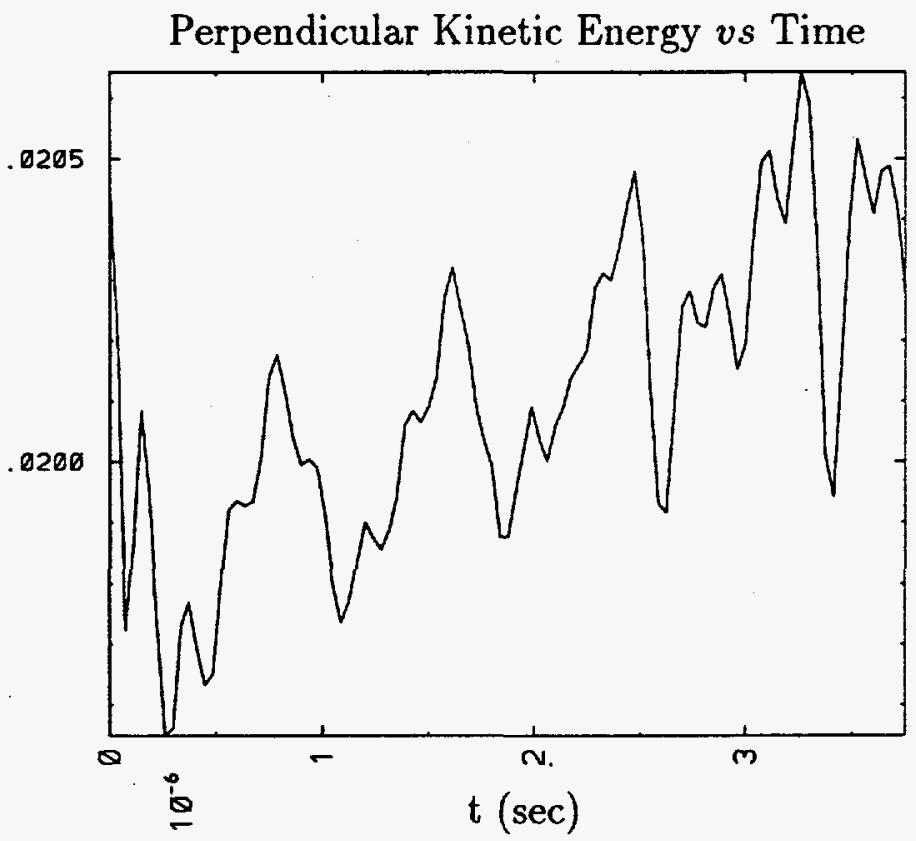

Figure 5.10: Although the field energy is decreasing, the increase in perpendicular kinetic energy is not large enough to compensate.

between the ion and electron is small. This is also true for our space charge waves. If we plot the $z$ kinetic energy of the particles as a function of time (figure (5.11)), the particle kinetic energy varies by the same amount that the field energy varies, but is out of phase. The fact that the wave causes these changes in the $z$ kinetic energy of the particles makes it difficult to see any increase in $z$ kinetic energy coming from the wave damping, especially since the amount of energy we are looking for is so small (.01 Joules). In fact, it may be impossible to trace this energy because it is so small compared to the total energy of the system (about .1\%). The total energy of the system (shown in figure (5.12)) is only conserved to about .1\%.

Although we don't have a neat and tidy explanation for the damping of the instability due to finite temperature effects, it is an important observation. We have seen the growth rate of the most unstable mode decreased by almost $50 \%$ when finite temperature is included in the system. We've also seen that high frequency, short 


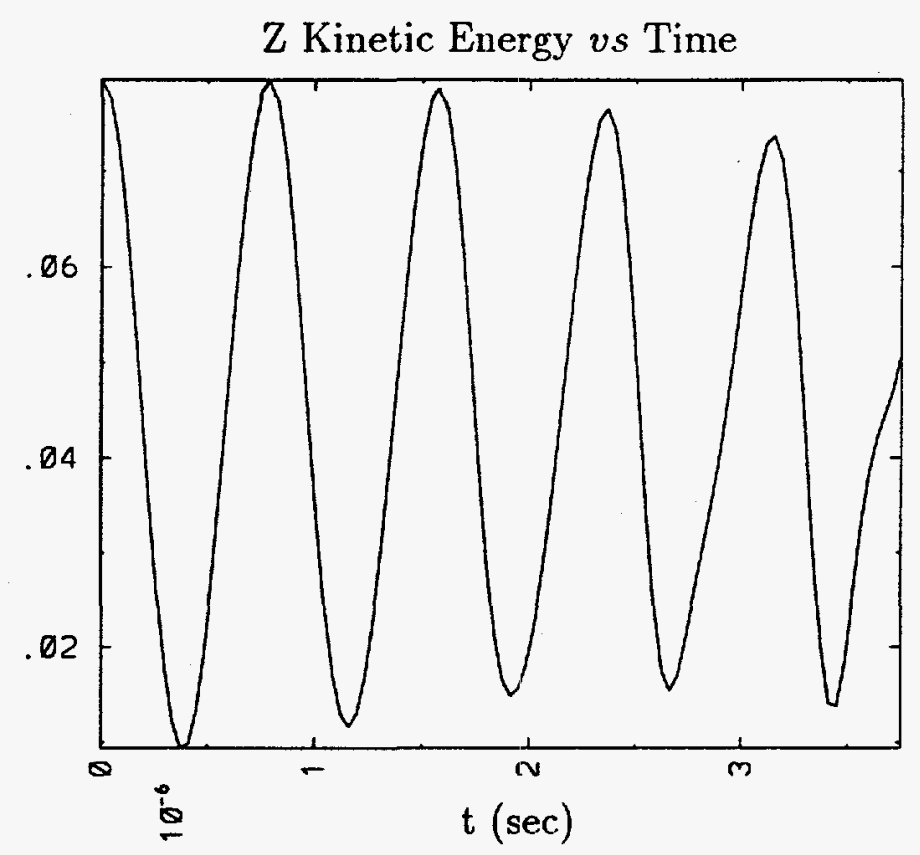

Figure 5.11: The energy of the wave is split between the field energy and the $Z$ kinetic energy.

wavelength perturbations undergo almost no growth at all. We have shown that finite longitudinal temperature alone does not explain the decrease in the growth rate observed in WARPrz. We have shown that damping of the longitudinal wave via transverse temperature is possible in the simple 1-d "wall and ball" model. We believe, however, in the 2-d WARPrz runs that energy is being coupled between the radial (transverse) and longitudinal directions. A complete two dimensional theory remains to be done. 


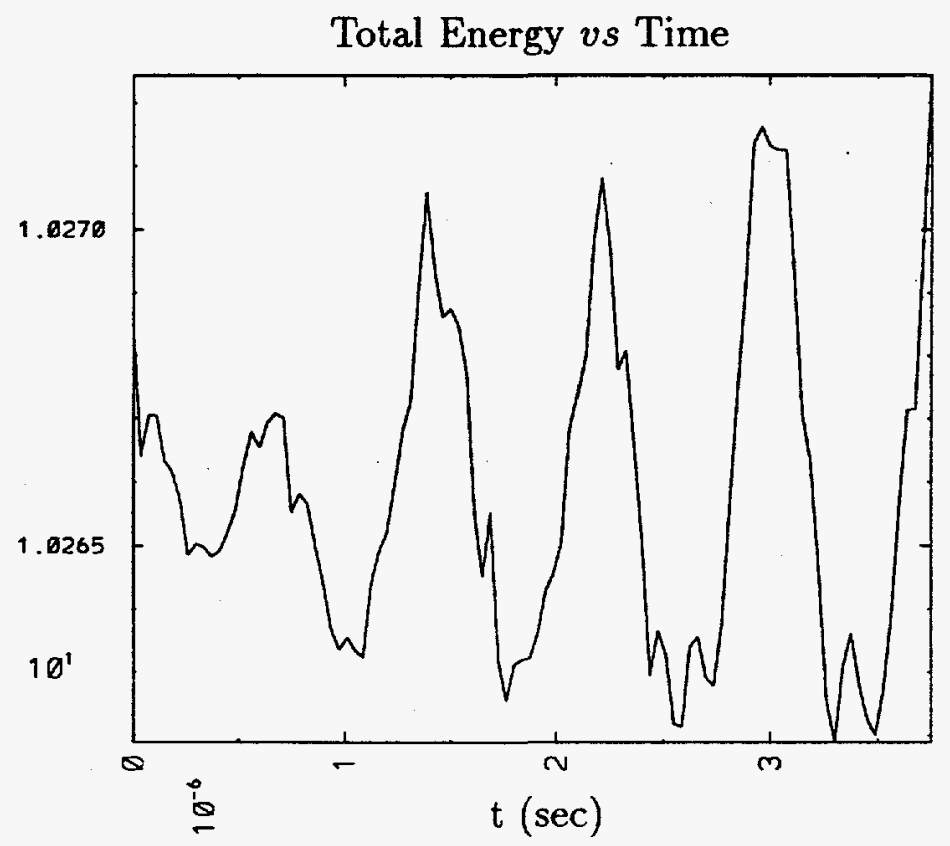

Figure 5.12: The total energy of the system is conserved to within .1\% but the amount of energy lost by the field energy is also about .1\% 


\section{Chapter 6}

\section{Intermittently-Applied Axial Confining Fields}

To get a realistic look at the effects of the longitudinal instability we need to consider not only the growth rate of the instability but also sources of finite amplitude perturbations which will be amplified. The intermittency of the axial confining fields ("ear" fields) is a leading candidate as a source of perturbations. In the simulations done in the previous sections, the ear fields used to keep the beam confined axially are applied at every time step and hold the beam at a constant length. In an accelerator, however, these fields will be applied at discrete intervals along the accelerator (e.g. every 100 meters). The beam will expand and contract axially as it travels down the accelerator. Each time the beam head is hit by the ear field, it may cause a perturbation to be launched in the beam and so we can picture a beam with a train of perturbations being launched from the head and traveling towards the tail. In this chapter, we discuss simulations of intermittently applied ear fields as a source of beam perturbations. 


\subsection{1-d Fluid Model}

We first study intermittently applied ears in the simpler 1-d fluid equations. Our mental picture of a beam with intermittently applied ears is a breathing beam. The beam expands freely when the ears are off and then is compressed by the ears fields. Since the beam will expand and contract, we use a Lagrangian representation for the fluid equations. In the Lagrangian representation, the grid moves with the fluid and is especially suited for problems of this type. The Lagrangian scheme used is given by the following set of equations:

Motion of the grid:

$$
z_{i}^{n+1}=z_{i}^{n}+u_{i}^{n} \Delta t
$$

Conservation of charge:

$$
\lambda_{i+1 / 2}^{n+1} \Delta z_{i+1 / 2}^{n+1}=\lambda_{i+1 / 2}^{n} \Delta z_{i+1 / 2}^{n}
$$

Conservation of momentum:

$$
\frac{u_{i}^{n+1}-u_{i}^{n}}{\Delta t}=\frac{q}{M}\left(E_{i}^{n}-g \frac{\lambda_{i+1 / 2}^{n}-\lambda_{i-1 / 2}^{n}}{\Delta z_{i}^{n}}\right)+\frac{F_{i}^{n}}{M}
$$

Circuit equation for a resistor:

$$
E_{i}^{n+1}=-\eta\left[\left(\frac{\lambda_{i+1 / 2}^{n+1}+\lambda_{i-1 / 2}^{n+1}}{2}\right)\left(u_{i}^{n+1}+u_{\text {beam }}\right)\right]
$$

where

$$
\begin{gathered}
\Delta z_{i+1 / 2}^{n}=z_{i+1}^{n}-z_{i}^{n} \\
\Delta z_{i}^{n}=\left(z_{i+1}^{n}-z_{i-1}^{n}\right) / 2
\end{gathered}
$$

and $F$ is the externally applied force which includes the ears fields.

Our first attempt at the ears fields was to apply a force which was a linear function of $z$ in the region which was expanding and was constant in time during the interval when the ears were "on". By varying the magnitude of the external force and the axial distance over which it was applied, we were able to get the error in velocity $u / v_{\mathbf{p}}$ down to about $1 \%$. When this was applied many times, the errors tended to add up 


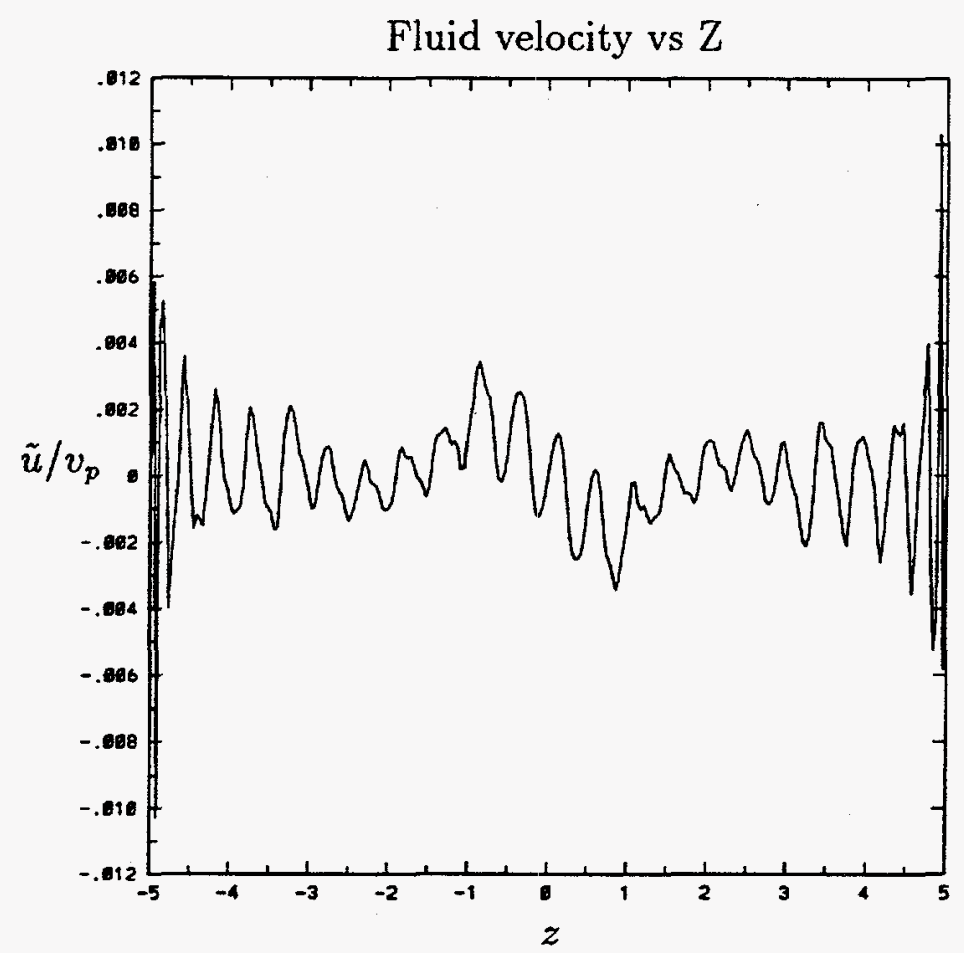

Figure 6.1: Fluid velocity (scaled to $v_{p}$ ) as a function of axial position after 10 applications of the ear fields

so that after 5 applications of the ears, the error was about $5 \%$. This would result in an error that was fairly large over the length of a driver.

We next tried the following. We make the applied force proportional to the integral over time of the force that was present during the free expansion stage $\left(t=0\right.$ to $t=t_{\text {on }}$ where $t_{\text {on }}$ is the time the ears are turned "on") -

$$
F(z)=-\hat{F} \int_{0}^{t_{\text {on }}} M \frac{d u(z, t)}{d t} d t=-\hat{F} M\left[u\left(z, t_{\text {on }}\right)-u(z, 0)\right]
$$

where $\hat{F}$ is a factor which we vary in order to get the minimum error. We apply the ears from time $t_{\text {on }}$ to $t_{\text {on }}+\tau_{\text {ears }}$. $\hat{F}$ should be approximately equal to $2 / \tau_{\text {ears. }}$. We assume that there is no velocity at time zero so that we can set $u(z, 0)=0$. We then save this external force and use the same force each time the ears are applied.

The motivation for using a force of this form is this: during the free expansion, the particles experience a particular force. If we were to apply the negative of that force back on the particles over the same length of time, the net force would be zero. 


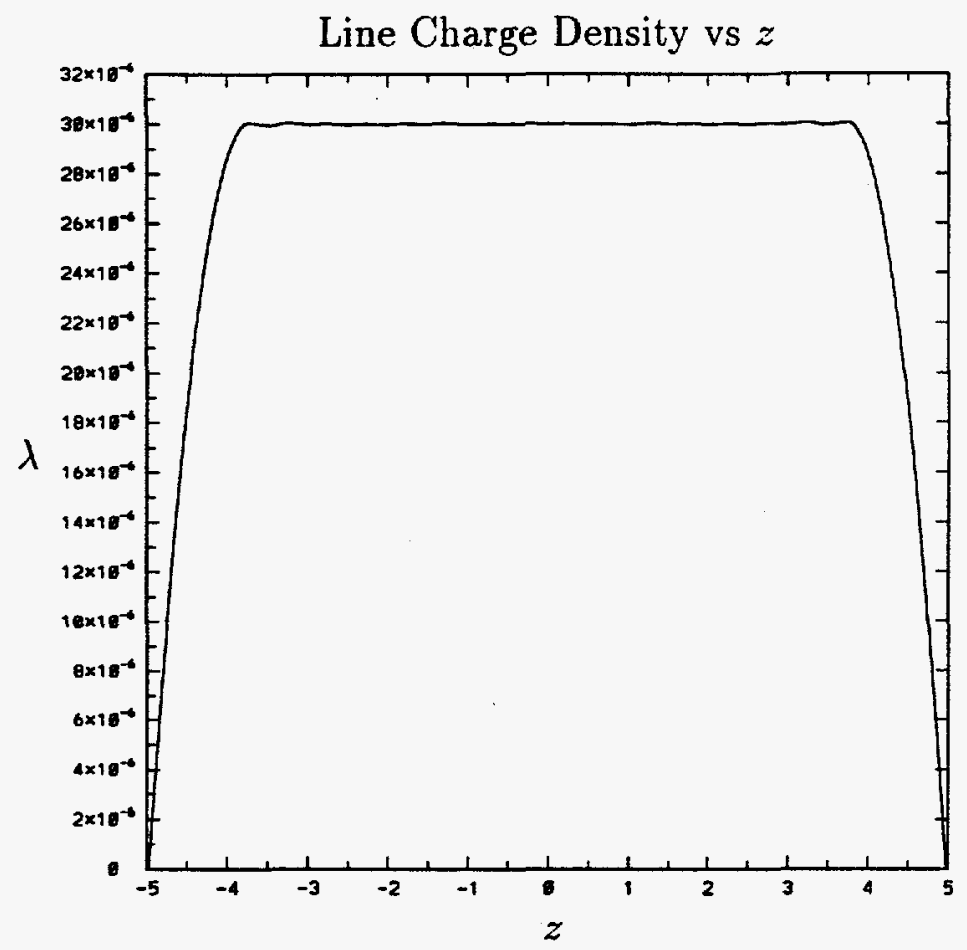

Figure 6.2: Line charge density as a function of axial position after 10 applications of the ears fields

What we'd like to do instead is to apply the same force over a shorter time interval (the interval during which the ear fields are turned on). Integrating the force applied during the free expansion stage gives us a kind of "total" force which is then applied back on the beam during the shorter time interval $\tau_{\text {ears. }}$. The factor of 2 in the $\hat{F}$ comes about because we want to reverse the expansion velocities.

We tested this on a beam which would be similar to a beam near the end of a driver. The ears fields were applied every 100 meters and kept on for a duration of 10 meters. The ear fields were applied to both ends of the beam simultaneously.

We then varied the parameter $\hat{F}$ until we minimized the error in velocity. This gave an error of approximately $0.2 \%$ after one application of the ears. After 10 cycles, however, the error in velocity was only $0.4 \%$. Figures $(6.1),(6.2)$ show the fluid velocity and line charge density after 10 applications of the ears fields. Figure (6.1) shows the error generated in the velocity (scaled to the wave phase velocity, $v_{p}$ ) is less than $1 \%$. In fact, the larger peaks near $z= \pm 1$ occur because the errors coming 


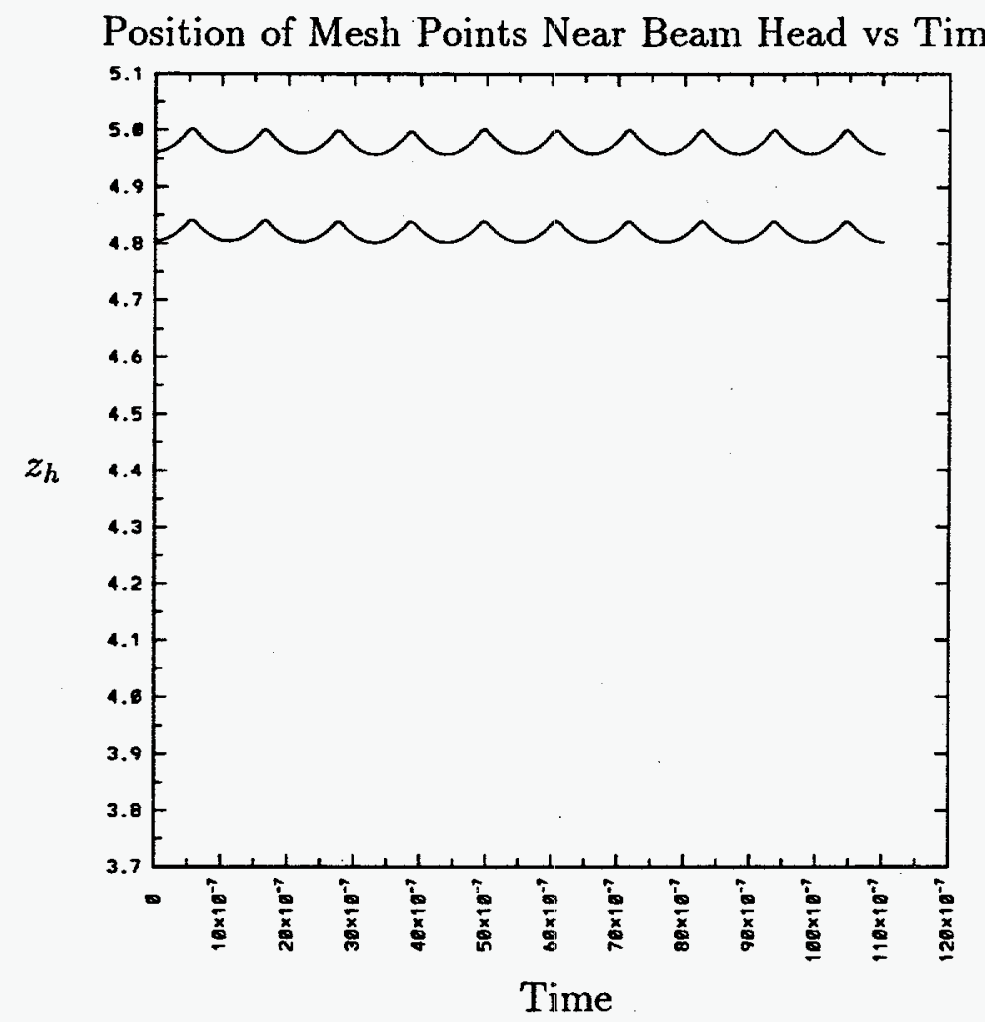

Figure 6.3: Position of grid points near the beam head shows the expansion and contraction of the beam.

off the beam head and tail have now overtaken each other and are constructively interfering. When resistance is included in the problem, the interference is a small effect because errors generated at the beam tail decay as they travel forward and become very small compared to the backward traveling waves, which grow. The line charge density is shown in figure (6.2). The tiny ripples are the errors.

Since we are using a Lagrangian scheme, the mesh expands and contracts along with the beam. In figure (6.3), we plot the position of the cell which is at the head of the beam. This shows the expansion and contraction of the head of the beam as a function of time. 


\subsection{Particle Simulations}

With the experience gained by using the 1-d fluid code under our belt, we move on to simulations of intermittent ears with the particle code. As our first attempt, we tried to apply the ears with as little damage to the beam as possible. To this end, we used the same form of the ears fields that we used in the fluid code-namely,

$$
F(z)=-\hat{F} \int_{0}^{t_{\text {on }}} M \frac{d u(z, t)}{d t} d t=-\hat{F} M u\left(z, t_{\text {on }}\right)
$$

where $\hat{F}$ is a factor which we vary in order to get the minimum error. In the particle code, the velocity, $u$, used to calculate the force was the average velocity of the particles as a function of $z$ in the beam frame.

As in the 1-d fluid runs, these fields are applied simultaneously to the head and tail of the beam. The ears fields are calculated on the same mesh we use to calculated the fields. This means during the short time that the ear fields are "on," they ride along with the beam. This is, of course, an approximation, since the fields will be applied at fixed locations in the lab frame in an experiment. This approximation is valid because the ear fields are on for such a short time for each beam particle that nothing can happen on this timescale. We have tested this with the WARPrz code by shortening the time the ears were on until it was only one or two timesteps (about .4 meters of distance). We found the results were the same as when the ears were applied for 10 meters.

In each of the runs in this series, we used parameters like those near the end of the accelerator section of the induction linac. In this portion of the accelerator, we have beam current of $3000 \mathrm{Amps}$, beam velocity of $.33 c$, pulse length of 10 meters. In our simulations, the beam pipe was $40 \%$ filled. For the simulations shown here we have again used the largest temperature that we can focus on the target (10 keV) to keep the amount of numerical heating small. This same temperature was used in both the perpendicular and parallel directions. A simulation with "perfect" ears (like the one described below) was done using a temperature of $2.5 \mathrm{keV}$ and gave the same results as as the $10 \mathrm{keV}$ case shown here. All of these simulations had a resistance of 100 
ohms/meter and no capacitance.

The ears were applied every 100 meters and kept on for 10 meters. Each cycle of the ear fields is made up of three parts- allow the beam to freely expand for 50 meters, apply the ear fields for 10 meters compressing the beam, then allow the beam to expand for another 50 meters. By choosing the cycle in this way, the beam should look the same at the end of the cycle as at the beginning. Figure (6.4) shows one cycle.

\section{Standard "perfect" ears run}

The first simulation in this series was an attempt to apply the intermittent ears with as little damage to the beam as possible. Figure (6.5) shows the potential vs axial position of the beam after 14 and 22 applications of the ears. By 14 applications, the perturbation launched at the beam head (the right hand side of the beam in our plots) has traveled about 7 meters and can be seen as a broad, shallow dip in the potential. After 22 applications, the beam looks similar. In this run, we do not see a trail of perturbations being launched off the beam head and growing towards the tail. The beam remains very smooth.

The electric field that we are applying in this case is fairly large. Figure $(6.7 \mathrm{a})$ shows the applied ear field as a function of $z$. The maximum electric field that we needed to apply every 100 meters to contain the beam is on the order of $17.5 \mathrm{MV} / \mathrm{m}$. This tells us that on average we need to apply $1.59 \mathrm{MV} / \mathrm{m}$ over the 110 meter cycle $((17.5 \mathrm{MV} / \mathrm{m}) \times 10 \mathrm{~m} / 110 \mathrm{~m})$. This is not acceptable because the total accelerating gradient that we have available is about $1 \mathrm{MV} / \mathrm{m}$ of which we'd like to use about $5 \%$ for confining the beam and $95 \%$ for accelerating it. All is not lost, however, because in our calculations, we have included the total beam current in one beam. In experiments, we will divide the current among multiple beams (16 for example) so that the confining fields are not so large. Calculations show that for 16 beams, the ear field is reduced by a factor $\sim 10-16$ depending on the location of the wall (which affects the $g$-factor). This tells us that at the end of the accelerator we need 
$v_{z} \operatorname{Vs} z$

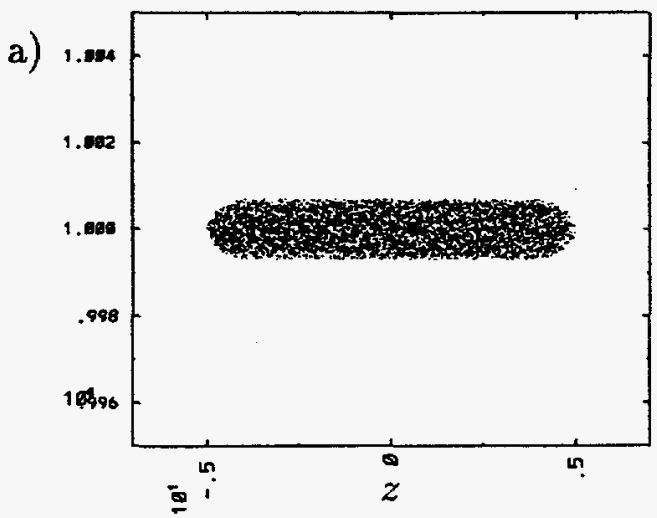

Ear Field vs $z$

c)

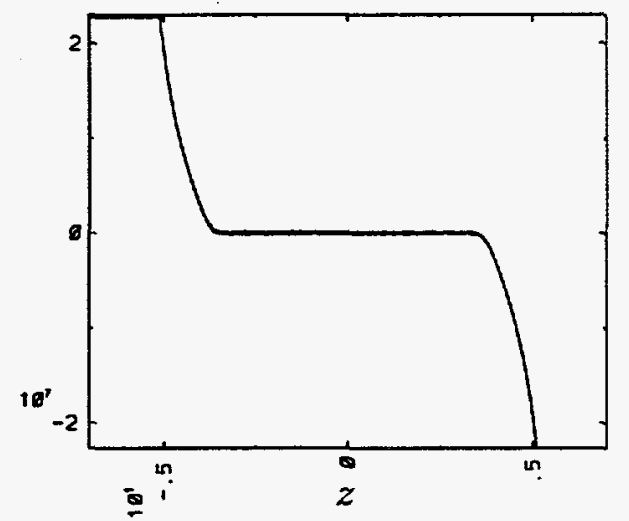

$v_{z}$ VS $z$

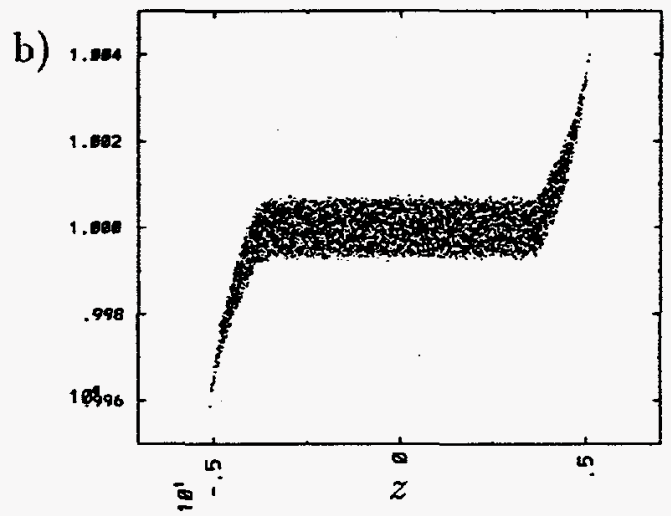

$v_{z}$ vs $z$

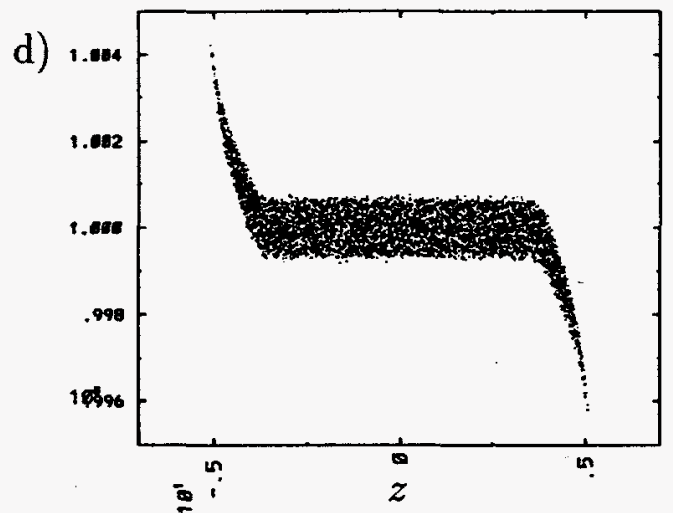

$v_{z}$ VS $z$

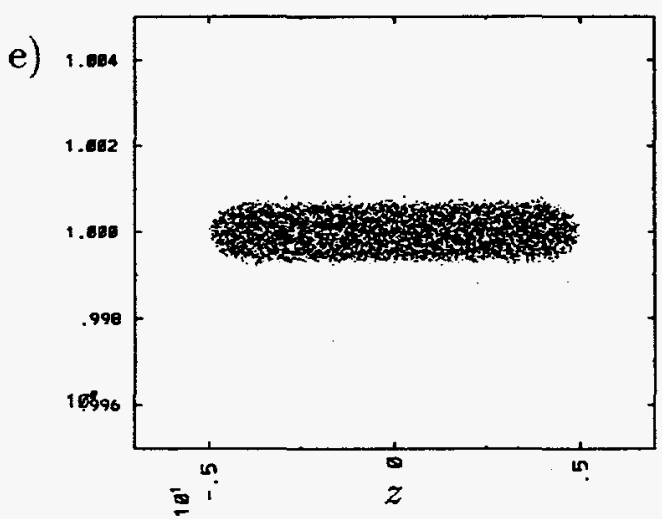

Figure 6.4: One cycle of intermittently-applied ears. (a) Initial phase space (b) Beam expands (c) Ear Field is applied (d) Beam is compressed (e) Beam expands back to its initial state 


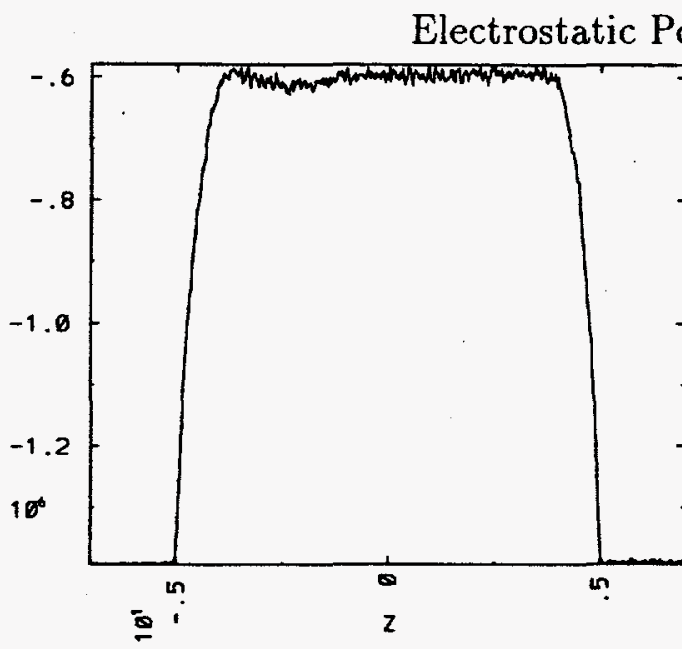

a) 14 applications

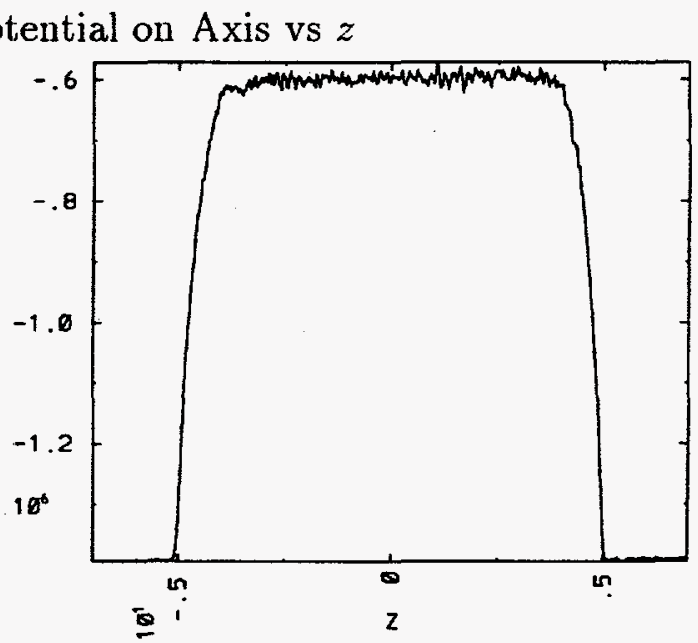

b) 22 applications

Figure 6.5: Applying "perfect" ears

to use $10-15 \%$ of the available accelerating field just to confine the beam. Techniques for reducing these fields further are being considered. It is comforting, however, to see that even though we are hitting the beam with a field like a sledgehammer, the beam behaves nicely.

A simulation like this one was done with a beam temperature of $2.5 \mathrm{keV}$ in both directions to look for any temperature effects. The results were the same as in the 10 $\mathrm{keV}$ case.

\section{"Perfect" ears applied less frequently"}

To get a handle on the effects of applying the ears too infrequently, we simulated the same situation as the "standard perfect" ears, but now we let the beam expand for 200 meters between ear applications. The ears were still applied over a distance of 10 meters. Figure $(6.6 \mathrm{a})$ shows the potential on axis after 7 applications of these ears. In this case, we see the perturbation that was launched by the first few sets of ears is nearing the tail of the beam and has grown fairly large.

Figure $(6.6 \mathrm{~b})$ shows the potential after 12 applications. We see the expected trail of perturbations being launched from the beam head and growing as they move towards the beam tail. Similar perturbations are probably being launched off the 
Electrostatic Potential on Axis vs $z$

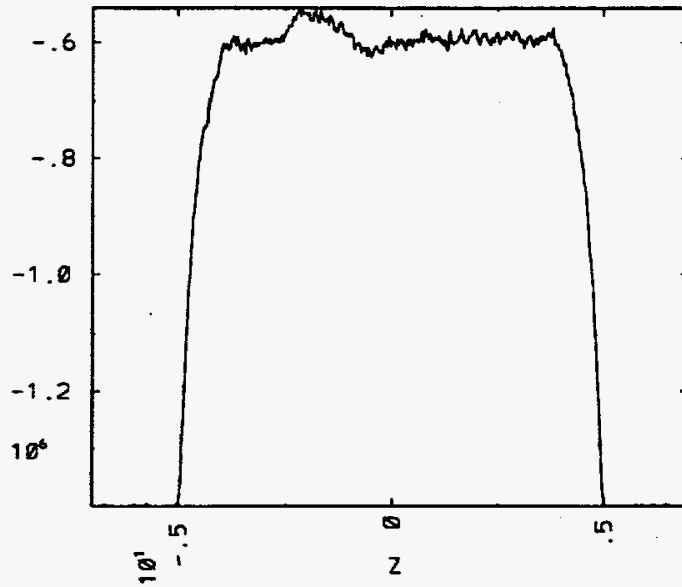

a) 7 applications

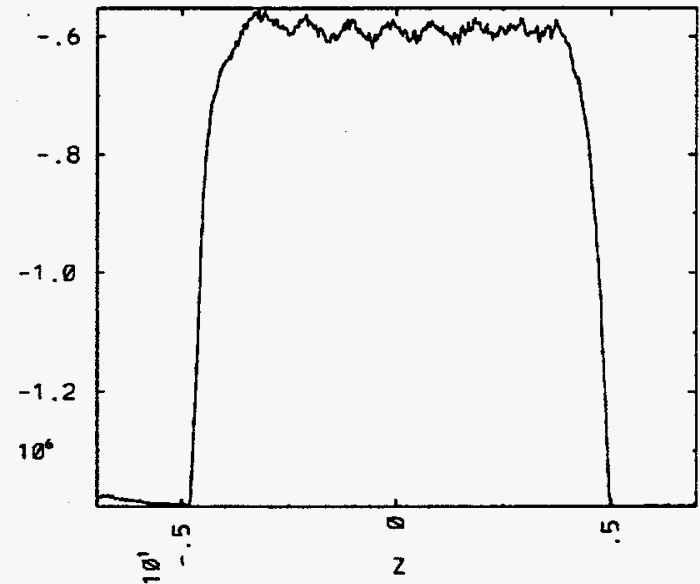

b) 12 applications

Figure 6.6: Applying ears every 200 meters excites a train of perturbations on the beam.

beam tail, however, they decay because they are forward propagating and so they are not seen. The wavelength of these perturbations is measured to be about 1 meter. If one perturbation were being launched with each application of the ears, we would expect that the wavelength would be approximately $\tau_{\text {ears }} v_{\text {phase }}$ where $\tau_{\text {ears }}$ is the time between ear applications and $v_{\text {phase }}$ is the wave phase velocity. In fact, this product yields a length equal to 1.02 meters.

\section{Imperfect Ears-ears too large}

Our first attempt at studying imperfect ears was to add a "bump" to the ear fields used in the case of perfect ears. Figure $(6.7 \mathrm{~b})$ shows the error added to the ears. The bump had the algebraic form of one half the period of a sine wave. This bump has the effect of making the ear fields too large. We applied these imperfect ears every 100 meters and left them on for 10 meters. We believed that by applying an error in the same direction every time, we would see a worst-case scenerio because there was no way for the errors to cancel one another out. We found that this was not a worst-case. 


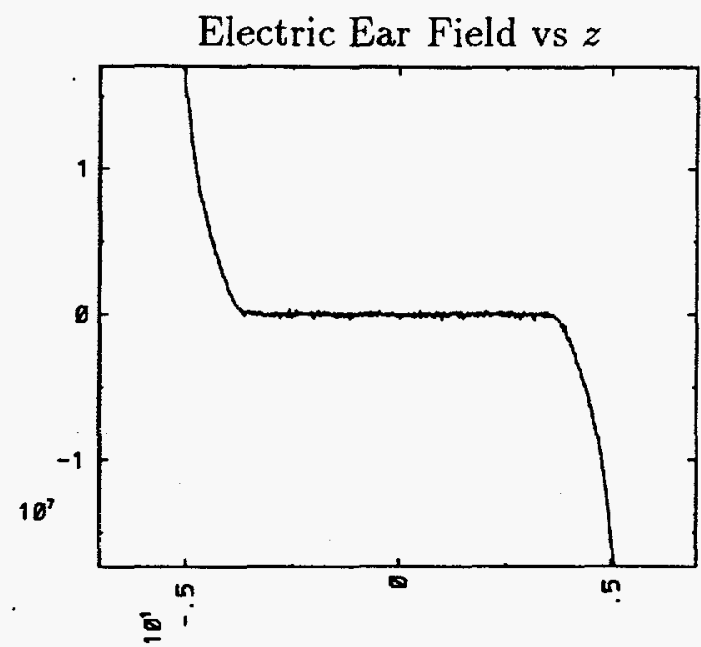

a) Ear Field (V/m)
Error Electric Field vs $z$

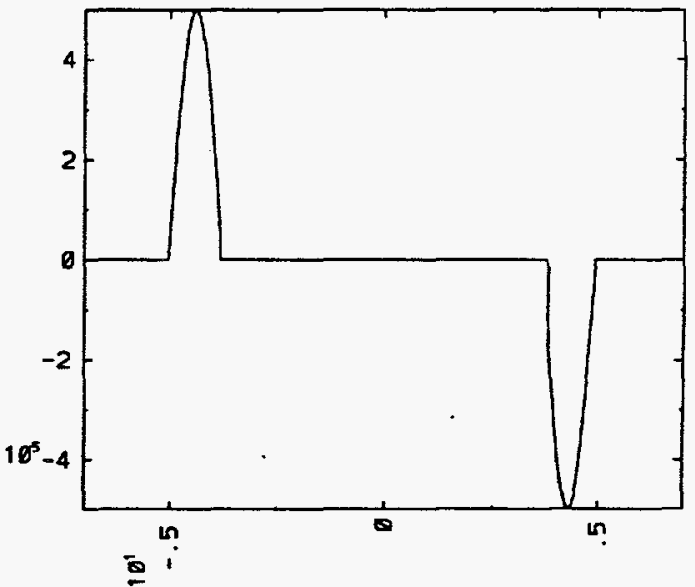

b) Error added to Ear field (V/m)

Figure 6.7: Ear field and error introduced

When we applied these ears, we found that the first few applications caused a perturbation to be launched from the beam head. Figure ( $6.8 \mathrm{a}$ ) shows this perturbation after 14 applications as it nears the beam tail after undergoing amplification by the longitudinal instability. Interestingly, if we look later in time (figure $(6.8 \mathrm{~b})$ ), we do not see a trail of perturbations coming off the beam head as we expect. In fact, the beam has adjusted itself to the error in the ears.

Electrostatic Potential on Axis vs $z$

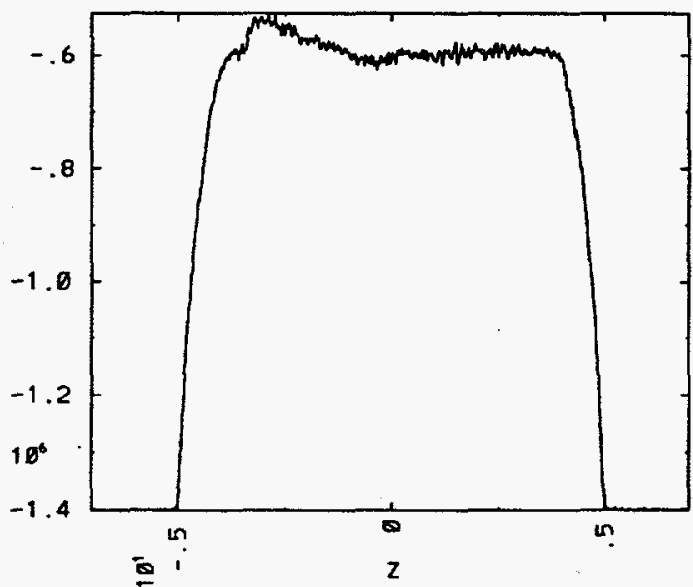

a) 14 applications

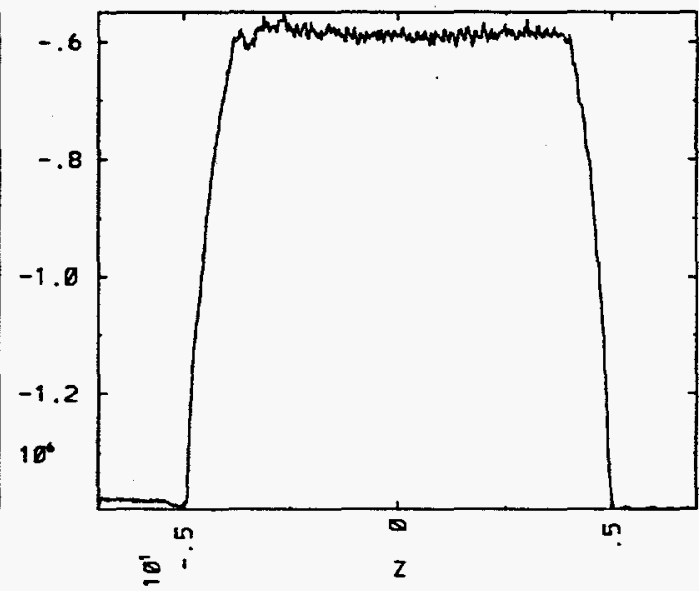

b) 22 applications

Figure 6.8: Applying ears that are always too large 
This phenomenon has also been seen in experiments done by A. Faltens at LBL[10]. These experiments were designed to test longitudinal bunch control in the beam tail in the single beam transport experiment (SBTE) at LBL. In these experiments, no attempt was made to match the waveform of the applied ear fields to the beam profile. Instead, fields with the waveform $1-\exp (-a t)$ were applied. They saw that mismatches in the ear fields caused waves to be launched from the beam tail in the early pulsers, but at later times the beam reached a new steady state configuration.

\section{Imperfect Ears-alternating too large and too small}

Once we found that the beam could adjust to an error in the ears that was always the same, we tried alternating the error. In this simulation, we applied the same size and shape error as in the previous run, but we alternated the sign of the error with each application. This amounted to applying ears that were too large on one application followed by ears that were too small on the next application.

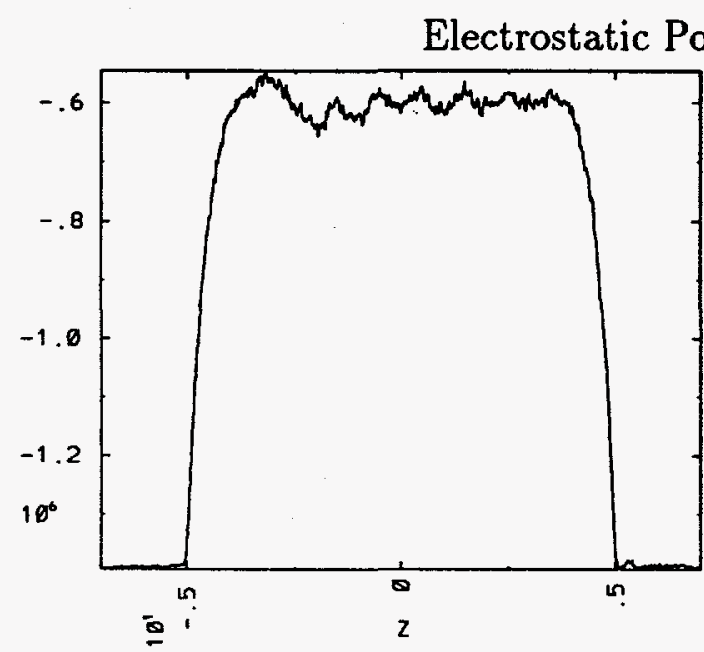

a) 14 applications

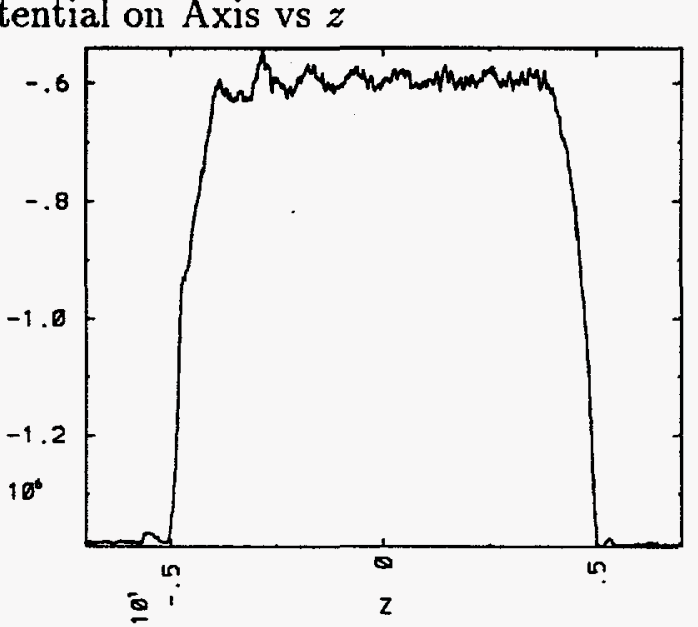

a) 22 applications

Figure 6.9: Applying ears that alternate too large and too small

Figure (6.9) shows that we generated the expected train of perturbations coming off the beam head and moving towards the tail. Again we expect a similar train of perturbations coming from the beam tail, but these perturbations decay as they travel towards the beam head and are not seen. The wavelength of the perturbations 
is measured to be about 1 meter. We calculate $2 \tau_{\text {ears }} v_{\text {plase }}=1.07$ meters. This indicates the cycle going on is:

1. hit the beam too hard and cause a enhancement region

2. this region moves away from the beam head in the time between ear applications

3. hit the beam too softly and cause a depletion region

4. this region moves away from the beam head in the time between ear applications

5. repeat

Such a cycle implies a perturbation wavelength of $2 \tau_{\text {ears }} v_{\text {phase. }}$

\section{Imperfect ears-an error of random size and sign}

An extension of alternating the sign of the error in the ear fields was to add an error of random size and sign. The error had the same shape as the previous two cases, but now the amplitude of the error varied randomly from $5 \%$ too large to $5 \%$ too small $(+5 \% \geq$ error $\geq-5 \%)$. Figure (6.10) shows the random variation of the ear fields as a function of application number. The result is the expected train of perturbations launched from the beam head and growing as they approach the tail. The width of the perturbations is measured to be approximately the wavelength of the most unstable mode of the longitudinal instability. Figure $(6.11 \mathrm{a})$ shows the electrostatic potential on axis vs $z$ after 15 applications of the ears, while figure $(6.11 \mathrm{~b})$ shows the same plots after 23 applications.

\subsection{Summary}

These simulations show how the intermittently applied ear fields and errors in the intermittently applied ear fields can generate perturbations on the beam which then grow via the longitudinal instability. The largest perturbations were generated when the amplitude of the error was allowed to vary randomly between $-5 \%$ and $+5 \%$. This 

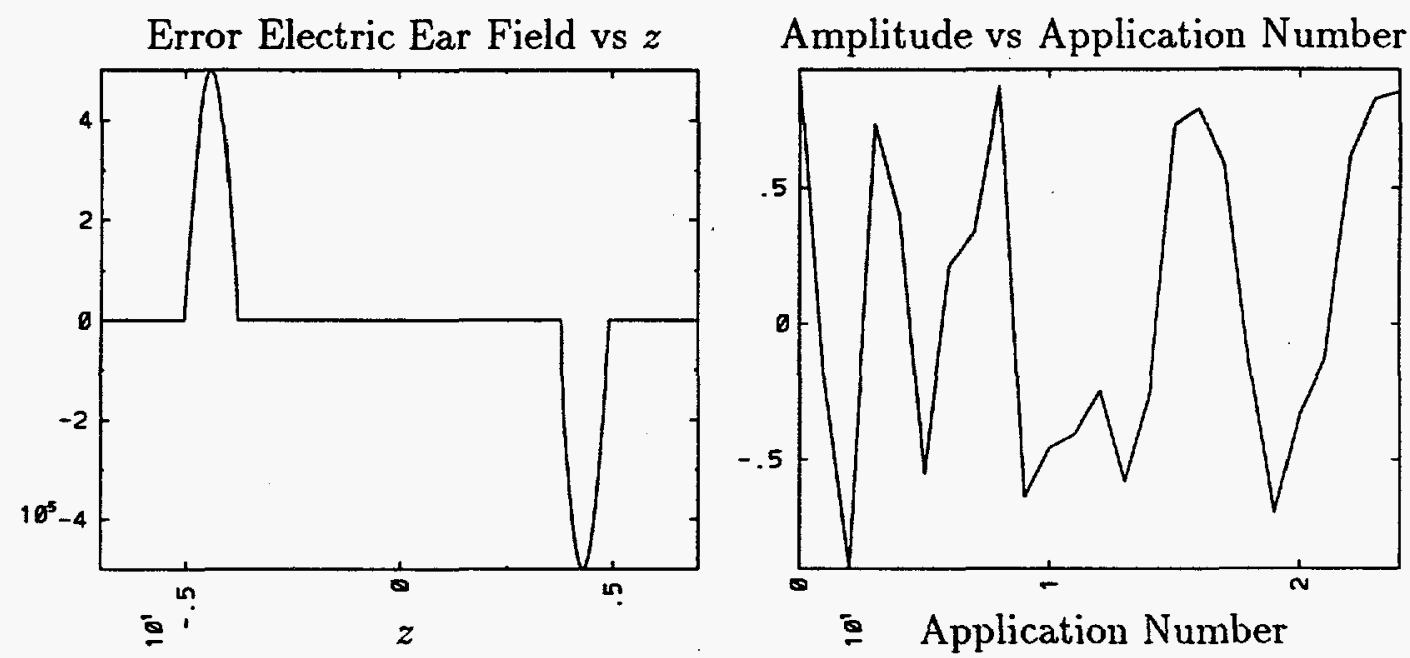

Figure 6.10: An error of random amplitude was introduced on each application by multiplying the error ear field by the random amplitude

excited the most unstable mode of the longitudinal instability and generated errors of $\Delta v / v_{p}$ of $15-20 \%$ in the presence of $100 \Omega / m$ resistance. This translates into a $\Delta p / p$ of $.073-.098 \%$ for $v_{p} / v_{\text {beam }}=4.88 \times 10^{-3}$. For final focus on the ICF target, $\Delta p / p \leq .1 \%$ at the end of the accelerator is needed.

These simulations did not include the effects of module capacitance. The simulations done in chapter 4 suggest that including the module capacitance would reduce the growth rate considerably and make the perturbations generated by errors in the intermittently applied ear fields even less severe. 
Electrostatic Potential on Axis vs $z$

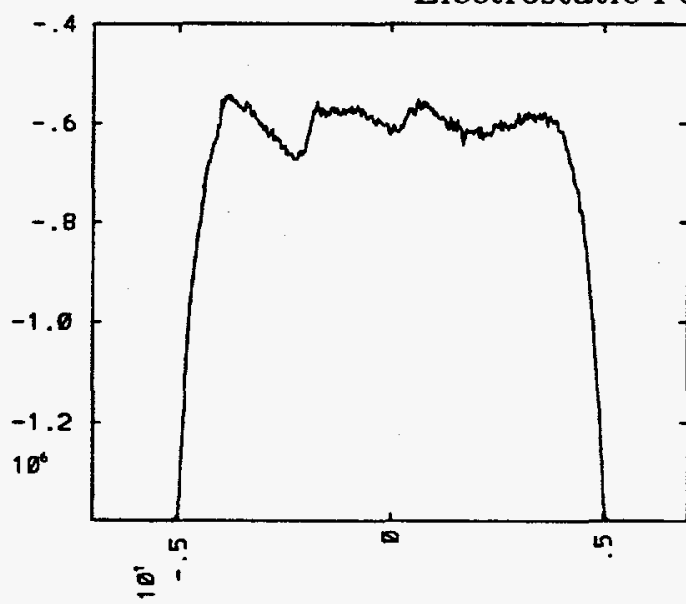

a) 14 applications

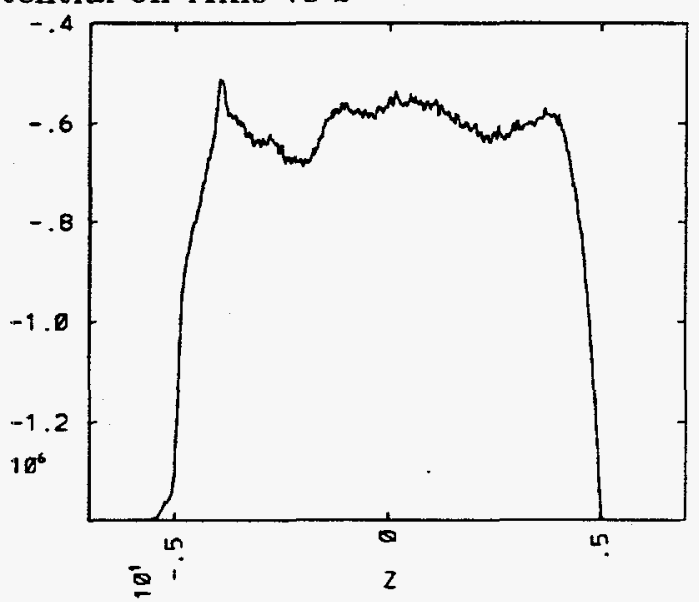

b) 22 applications

Figure 6.11: A random error in the ears excites the most unstable wavelength of the longitudinal instability 


\section{Chapter 7}

\section{Conclusions and Future Work}

\subsection{Conclusions}

For more than 10 years, the longitudinal instability has been a threat to the success of heavy ion driven inertial confinement fusion for energy production. Early calculations included only the resistive part of the module impedance and predicted 10's of $e$ foldings of growth. This would be disastrous because chromatic aberrations in the magnetic lens focusing system cause particles with different longitudinal velocities to be focused at different locations. Thus, large perturbations in longitudinal velocity decrease the power delivered to the target.

The low growth rate of this instability makes it difficult to study. In present day ion beam experiments, the instability is not seen because the amount of growth is too small. The University of Maryland group will attempt to increase the growth rate of this instability by passing a space-charge dominated electron beam through a glass tube with a high resistance coating. However, theory predicts an increase in wave amplitude of only about $25 \%$ in the UM experiment because the resistive tube is only 1 meter long. This instability must be understood before a heavy ion fusion driver can be built.

In this thesis, I have studied the longitudinal instability using an axisymmetric particle-in-cell code. This code includes a model for the impedance of the accelerating 
modules as a continuum of resistors and capacitors in parallel. Using this code, we have simulated ion beams over several kilometers and observed growth from this instability. We find that growth is mainly limited to the growth that occurs as a beam perturbation travels from head to tail. Some additional growth can occur on successive passes if the perturbation steepens during reflection off the beam tail.

Cold beam theory predicts the capacitive component of the module impedance partially stabilizes the longitudinal instability. The capacitive component of the impedance was neglected in the early calculations and plays an important role in the effect of the instability on an HIF driver. Our simulations confirm that including capacitance decreases the growth rate considerably.

It has long been known that longitudinal temperatures which are acceptable for the focusing system have little effect on the longitudinal instability. A $20 \%$ decrease in the growth rate from longitudinal temperature requires a longitudinal thermal speed more than three times what is acceptable in the magnetic focusing system.

Our two dimensional simulations have shown decreases in growth rate of $20 \%$ or more even with longitudinal temperatures acceptable for the focusing system. This decrease in growth rate is due to transverse temperature which was neglected in previous calculations. In chapter 5 , we propose a simple mechanism for coupling transverse thermal motion to the observed decrease in growth rate. From this model, we expect that acceptable levels of transverse temperature can decrease the growth rate of the longitudinal instability.

We have also investigated sources of beam perturbations. The most likely source of perturbations is the "ear" fields used to confine the beam ends. The voltages required to produce the ear fields will have fairly complicated waveforms and must be timed correctly in order to keep the beam confined and prevent large perturbations from being launched. These perturbations are amplified by the longitudinal instability.

Our simulations have shown that ear fields can be applied every 100 meters without generating large perturbations on the beam. Adding a systematic $5 \%$ error to the ear fields generates a perturbation from the first few ear applications, but the beam is 
able to adjust to the error. This is important for the HIF program because it means that the waveforms used to generate the ear fields do not need to be closely matched to the beam profile. This has also been seen in experiments at Lawrence Berkeley Laboratory.

Adding a random $5 \%$ error to the ear fields excites the most unstable mode of the longitudinal instability. The simulations shown in chapter 6 do not include the capacitive component of the module impedance. From our earlier simulations with and without capacitance, we believe that the capacitive component will reduce the growth these perturbations considerably.

The longitudinal instability is no longer a threat to the heavy ion fusion program. The simulations in this thesis have shown that the instability will not be as severe as was once thought. We have shown that growth is mostly limited to one pass from beam head to tail and that the capacitive component of the module impedance reduces the growth rate of the instability considerably. In addition, we have shown that transverse temperature can also decrease the growth rate of the instability. The longitudinal instability is no longer a "show stopper" for the heavy ion fusion program.

\subsection{Future Work}

Once the longitudinal instability experiment at the University of Maryland is operational, we will compare these experimental results with the WARPrz results. Although comparisons between WARPrz and other electron beam experiments at UM have been successful[13], this will be the first experimental confirmation of the longitudinal instability results from WARPrz.

One improvement we would like to make to WARPrz is to add a discrete model for the accelerating gaps. This will allow us to study accelerating beams and the effect of errors in the accelerating fields on the beam. These gaps will be used to supply both the main accelerating pulse and the ear fields as will be the case in experiments. Adding accelerating gaps will allow us to improve our understanding of allowable 
tolerances in errors in the voltages applied to the gaps and will give us a closer tie with experiment.

We plan to use WARPrz for simulations other than those relating to the longitudinal instability. Near the end of the accelerator, the pulse must be shaped with a long, low "foot" required by the ICF target designs. This shaping can be done by changing the velocity profile of the beam such that the beam drifts into the correct shape, or by stacking pulses which have traveled through different path lengths, or some combination of both. We plan to model this pulse shaping with WARPrz.

We will use WARPrz to continue studying an instability which causes rapid heating in the longitudinal direction when the beam is initially much colder in the longitudinal direction than in the perpendicular direction. This mode appears to be similar to the Harris mode in magnetized plasmas[15, 23]. This instability has been seen in both WARPrz and WARP3d simulations[12] and may limit the amount of temperature anisotropy allowed in heavy ion fusion driver beams.

We also plan to use WARPrz as part of the HIF source-to-target modeling project. This project will use a variety of codes to model a beam through the entire HIF driver, into the reactor chamber, and finally onto an ICF target. The majority of these simulations will need to be done in 3 dimensions using WARP3d, but WARPrz will be used when 3-d effects are not important because of its speed. 


\section{Appendix A}

\section{List of Symbols}

Quantities used in this thesis and in WARPrz are in MKS units. Bold face indicates a vector quantity while scalars are set in italics.

$\begin{array}{ll}a & \text { Beam radius } \\ b & \text { Pipe radius } \\ A_{i} & \text { Areas used for interpolating particle data to the grid } \\ \mathbf{B} & \text { Magnetic field } \\ c & \text { Speed of light in vacuum } \\ \mathcal{C} & \text { Module capacitance (units of Farad-meters) } \\ e & \text { Elementary charge } \\ \mathbf{E} & \text { Electric field } \\ f & \text { Distribution function } \\ \mathbf{F}_{\text {magnetic }} & \text { Force on a particle due to the magnetic field } \\ \mathbf{F}_{\text {electric }} & \text { Force on a particle due to the electric field } \\ g & \text { Geometric factor (defined in eqn } 1.10) \\ I_{b} & \text { Beam current } \\ \delta I_{b} & \text { Perturbed beam current } \\ I_{0}(z) & \text { Initial current profile as a function of } z \\ I_{0}, K_{0} & \text { Modified Bessel functions } \\ I_{1}, K_{1} & \text { Modified Bessel functions } \\ J_{0}, N_{0} & \text { Bessel functions } \\ J_{1}, N_{1} & \text { Bessel functions } \\ k & \text { Wave number } \\ K_{z} & \text { Surface current on the wall } \\ \mathcal{L} & \text { Module inductance (units of henries/meter) } \\ M & \text { Ion mass } \\ q & \text { Ion charge } \\ Q_{j, k} & \text { Charge at gridpoint }(j, k)\end{array}$




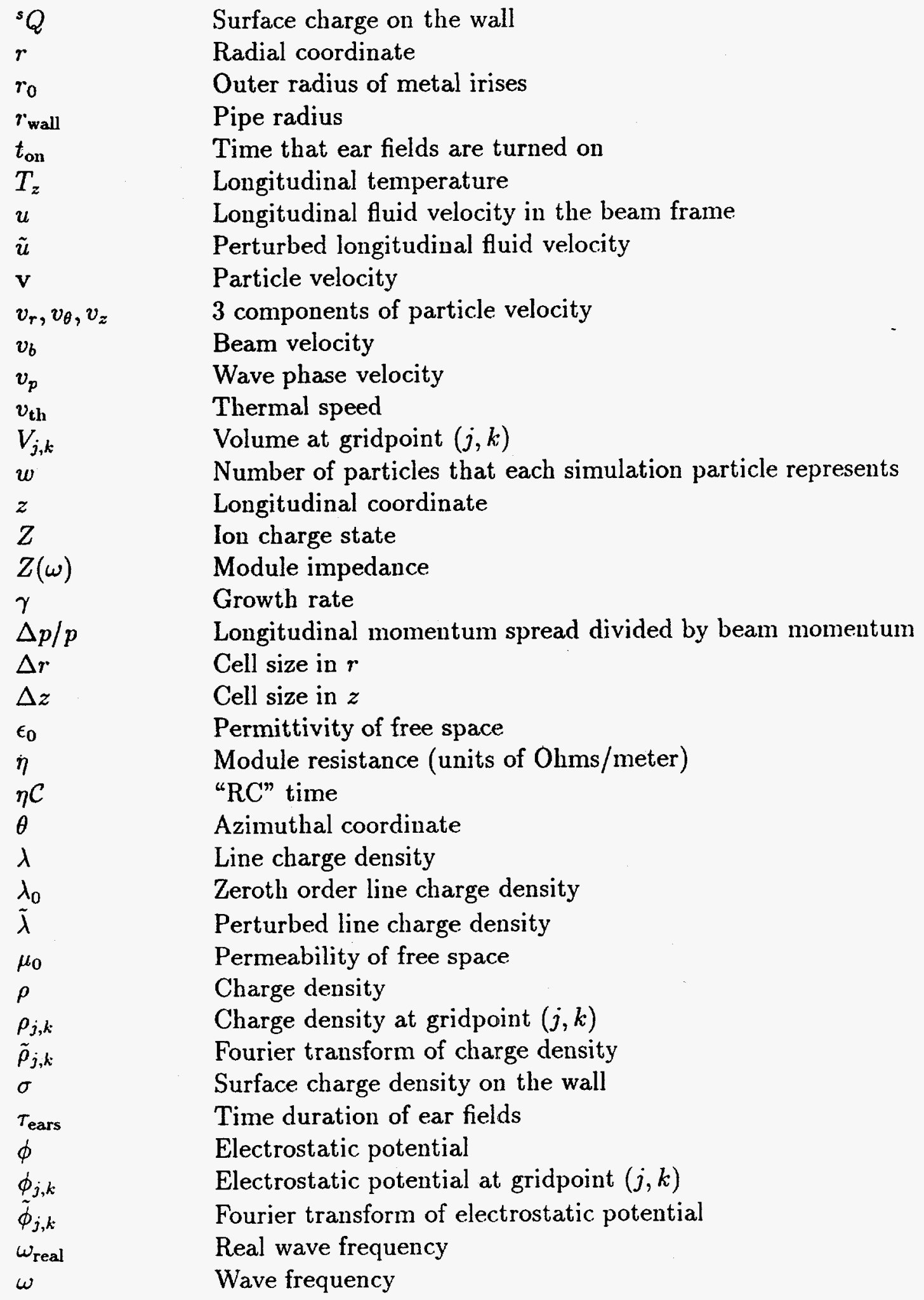




\section{Bibliography}

[1] R. T. Avery "Program Plan for the MBE-4 Multiple Beam Experiment," LBL-19592, February 1985.

[2] J. J. Barnard, F. Deadrick, A. Friedman, D. P. Grote, L. V. Griffith, H. C. Kirbie, V. K. Neil, M. A. Newton, A. C. Paul, W. M. Sharp, H. D. Shay, R. O. Bangerter, A. Faltens, C. G. Fong, D. L. Judd, E. P. Lee, L. L. Reginato, S. S. Yu, and T. F. Godlove, Physics of Fluids B, 5, 2698, (1993).

[3] C. K. Birdsall, G. R. Brewer, A. V. Haeff, Proc. of the I.R.E., 41, July 1953.

[4] C. K. Birdsall and A. B. Langdon, Plasma Physics via Computer Simulation, McGraw-Hill, New York, 1985.

[5] J. Bisognano, I. Haber, L. Smith, IEEE Transactions on Nuclear Science, NS-30, 2501, (1983).

[6] J. Bisognano, I. Haber, L. Smith, and A. Sternlieb, IEEE Transactions on Nuclear Science, NS-28, 2513, (1981).

[7] N. Brown, M. Reiser, D. Kehne, D. X. Wang, J. G. Wang, Proc. of IEEE/APS Conference on Particle Accelerators, Washington DC, May 17-20, 1993.

[8] R. C. Davidson, Theory of Nonneutral Plasmas, Addison-Wesley, Menlo Park, CA, 1989.

[9] S. Eylon, et al., "Transverse Beam Dynamics Studies with the MBE-4 Multiple Beam, Heavy-Ion Linear Accelerator," in preparation.

[10] A. Faltens, LBL Half Year Report LBL-19501, June 1985.

[11] T. J. Fessenden and A. Friedman, Nuclear Fusion, 31, 1567 (1991).

[12] A. Friedman, D. A. Callahan, D. P. Grote, A. B. Langdon, and I. Haber, Bull. Am. Phys. Soc., Cincinnatti, OH, 1990. 
[13] I. Haber, D. A. Callahan, A. B. Langdon, M. Reiser, D. X. Wang, J. G. Wang, Proc. of IEEE/APS Conference on Particle Accelerators, Washington, DC, May 17-20, 1993.

[14] K. Hahn, Bull. Am. Phys. Soc., 37, 1414, (1992).

[15] E. G. Harris, Phys. Rev. Letters, 2, 34, (1959).

[16] I. Hoffman and I. Bozsik, Proc. of the Symposium on Accelerator Aspects of Heavy Ion Fusion, Darmstadt, W. Germany, 1982.

[17] E. J. Horowitz, Journal of Computational Physics, 68, 56, (1987).

[18] A. B. Langdon, private communication, 1993.

[19] E. P. Lee, Particle Accelerators, 37-38, 307, 1992.

[20] E. P. Lee, Proc. Linear Accelerator Conference, (UCRL-86452), Santa Fe, NM, 1981.

[21] D. Neuffer, IEEE Transactions on Nuclear Science, NS-26, 3031, (1979).

[22] M. Reiser and J. G. Wang, Proc. of the Workshop on the Longitudinal Instability, E. P. Lee editor, HIFAN-540, Lawrence Berkeley Laboratory, February 26-27, 1992.

[23] G. K. Soper, and E. G. Harris, Phys. Fluids, 8, 984, (1965).

[24] D. X. Wang, J. G. Wang, D. Kehne, M. Reiser, I. Haber, Proc. of IEEE/APS Conference on Particle Accelerators, Washington, DC, May 17-20, 1993.

[25] D. X. Wang, J. G. Wang, D. Kehne, M. Reiser, I. Haber, International Symposium on Heavy Ion Fusion, Frascati, Italy, May 25-28, 1993.

[26] J. G. Wang and M. Reiser, Phys. Fluids B, 5, 2286, (1993).

[27] J. G. Wang, D. X. Wang, D. Kehne, M. Reiser, Proc. of IEEE/APS Conference on Particle Accelerators, Washington, DC, May 17-20, 1993.

[28] J. G. Wang, D. X. Wang, D. Kehne, M. Reiser, H. Suk, International Symposium on Heavy Ion Fusion, Frascati, Italy, May 25-28, 1993.

[29] S. S. Yu, J. J. Barnard, G. J. Caporaso, A. Friedman, D. W. Hewett, H. Kirbie, M. A. Newton, V. K. Neil, A. C. Paul, L. L. Reginato, W. M. Sharp, T. F. Godlove, R. O. Bangerter, C. G. Fong, and D. L. Judd, Particle Accelerators 37-8, 489 (1992). 